\title{
Information Processing Analysis in Neural Networks
}

\author{
Dissertation \\ for the award of the degree \\ "Doctor rerum naturalium" \\ of the Georg-August-Universität Göttingen \\ within the doctoral program GGNB \\ of the Georg-August University School of Science (GAUSS) \\ submitted by \\ Chenfei Zhang \\ from Shaanxi, P. R. China \\ Göttingen, 2019
}


Thesis Committee

Prof. Dr. Fred Wolf

Max Planck Institute for Dynamics and Self-Organization

Prof. Dr. Jörg Enderlein

Third Institute of Physics, University of Göttingen

Dr. Robert Gütig

Max Planck Institute of Experimental Medicine

Members of the Examination Board

Reviewer: Prof. Dr. Alexander Gail

German Primate Center, Göttingen

Second Reviewer: Prof. Dr. Siegrid Löwel

Faculty of Biology, University of Göttingen

Additional Reviewer (if applicable): Dr. Marion Silies

European Neuroscience Institute Göttingen

Date of the oral examiniation: June $7^{\text {th }}, 2018$ 
Herewith I declare, that I prepared the PhD Thesis

"Information Processing Analysis in Neural Networks"

on my own and with no other sources and aids than quoted.

Göttingen, 2019

Chenfei Zhang 


\section{Contents}

1 Introduction 3

1.1 Brain . . . . . . . . . . . . . . . . . . . 3

1.2 Action Potential . . . . . . . . . . . . . . 3

1.2.1 Action Potential Generation Mechanisms . . . . . . 3

1.2 .2 Action Potential Onset Rapidness . . . . . . . . . . 3

1.3 Fast Information Processing in the Brain. . . . . . . . . . 6

1.4 Fast Population Encoding . . . . . . . . . . . . . . . . . 7

1.4.1 Fluctuation-Driven Firing and Weakly Correlated

Cortical Neurons . . . . . . . . . . . . . . . 7

$1.4 .2 \quad$ High Bandwidth Encoding _... . . . . . . . . . 11

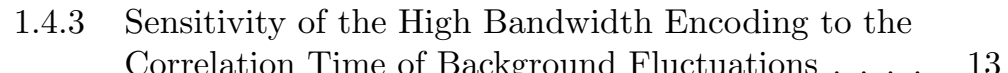

1.5 Theoretical Approaches on Population Encoding . . . . . . 14

1.5.1 Ultrafast Population Encoding and Realistic Neuron Modeling ....................... 16

1.5.2 The Impact of AP Onset Rapidness on Population Encoding ................... 17

1.5.3 Fokker-Planck Approach and Gauss-Rice Approach to Population Encoding . . . . . . . . . . . . 18

$1.6 \quad$ Three Hypotheses on the Impact of Neuron Morphology and Biophysics on Population Encoding . . . . . . . . . . . . . . . . 21

$1.6 .1 \quad$ Axonal Hypothesis . . . . . . . . . . . . . . . . . . 21

1.6.2 Cooperative Gating of Sodium Channels Hypothesis 22

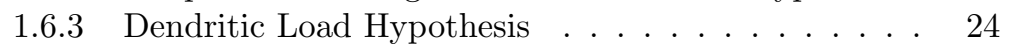

1.7 A Brief Summary of My Work . . . . . . . . . . . 26

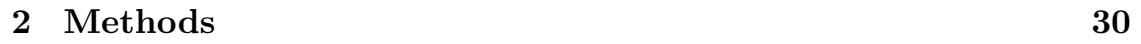

2.1 Introduction to Brette's Model . . . . . . . . . . . . . . . 30

2.2 Simulation Setup and Parameter Searching . . . . . . . . . 32

2.3 Two Methods for Calculating Linear Response Functions . 34

2.4 Bootstrapping and Null Hypothesis Testing . . . . . . . . . 39

2.5 Evaluation of the AP Initiation Dynamics on Population

3 The Impact of AP Initiation Site Location on Fast Population Encoding 53

3.1 The Impact of AP initiation Site on Population Encoding . 53

3.2 The Impact of High Voltage Sensitivity of Sodium Channels on Population Encoding . . . . . . . . . . . . . . 57

3.3 Somatic and Axonal Voltage Dissociation . . . . . . . . . 60

3.4 Simplified Model Representation . . . . . . . . . . . . . . . 62

$3.5 \quad$ Discussion $\ldots \ldots \ldots \ldots \ldots$. . . . . . . . . . . . . . . . . . 64

\begin{tabular}{|lll}
\hline & The Implications of High Voltage Sensitivity & 67
\end{tabular}

4.1 Population Encoding of the Cooperative Gating Model . . . 67

4.2 Examination of the Cooperative Gating Model $\ldots$. . . . . 70

4.3 The Impact of AP Initiation Site Location and Soma Size on Population Encoding . . . . . . . . . . . . . . 78 
5 High Bandwidth Encoding with High Frequency Repetitive Firing

83

5.1 Introduction to Eyal's Model . . . . . . . . . . . . . . . 83

5.2 The Mechanisms for High Frequency Repetitive Firing . . . 89

$\begin{array}{lll}6 & \text { Summary } & 94\end{array}$ 


\section{Introduction}

\section{$1.1 \quad$ Brain}

The brain is the core of information processing for humans. With billions of neurons and tens of thousands of connections for each neuron, the brain is in charge of a diversity of functional roles ranging from the sensory and motor control to higher level learning and decision making.

Understanding the underlying mechanisms of how the brain realizes such complicated functions is one of the most difficult questions. One line of research starts with the behavioral experiments and tries to identify the brain areas responsible for corresponding behaviors. Another line of research starts with the dynamics of individual neurons and tries to identify the neural circuits and the network connections responsible for the functional roles. In this thesis, I will take the second approach and study the impact of individual neuron properties on the action potential generation dynamics and the encoding ability of the neuron populations.

\subsection{Action Potential}

\subsubsection{Action Potential Generation Mechanisms}

Action potentials are the major communication signals between cortical neurons. Thousands of synaptic inputs lead to the continuous voltage fluctuations in one neuron. Small fluctuations can not be passed down and detected by the downstream neurons. Only large enough fluctuations which trigger action potentials in the axon can be "known" by the other neurons. In this sense, large amounts of input information are processed in the neuron and compressed into discrete delta pules as the output. Understanding how the information is integrated in the neuron, how the spikes are generated, and the impact of spike generation dynamics on the neuron capability contribute to unveiling the information encoding mechanisms of cortical neurons

The early works of Hodgkin and Huxley [1-5] characterized the ionic mechanisms of action potential initiation and proposed a conductance-based model to describe the action potential generation dynamics. An action potential is triggered by a surge of sodium current entering the neuron. When the voltage is high enough, the sodium current is reduced by the inactivation of sodium channels and the voltage is brought down by the potassium current. With the deinactivation of sodium channels, the sodium current becomes available again for the next spike.

The conductance-based model proposed by Hodgkin and Huxley provides a biophysical plausible framework for explaining action potential generation. The neuron model is a type II model. In a subsequent work of [6, type I conductance-based models were also developed.

\subsubsection{Action Potential Onset Rapidness}

In 7, the authors pointed out two potential problems of conductance-based models as applied to cortical nerve cells. As shown in Fig 1 for the spikes recorded in cortical neurons, there is a kink at the initiation of a spike. From the view of a phase plot, the voltage derivative rises rapidly in a short voltage interval at the beginning of the spike. Observations above are valid both in vivo and in vitro. However, for conductance-based models, there is 
no kink at the initiation of spikes. The voltage derivative rises smoothly with the voltage in its phase plot. The other potential problem is the variation of the spike threshold. For cortical neurons, the spike threshold has a broad range. However, for the conductance-based model, the spike threshold range is quite narrow.
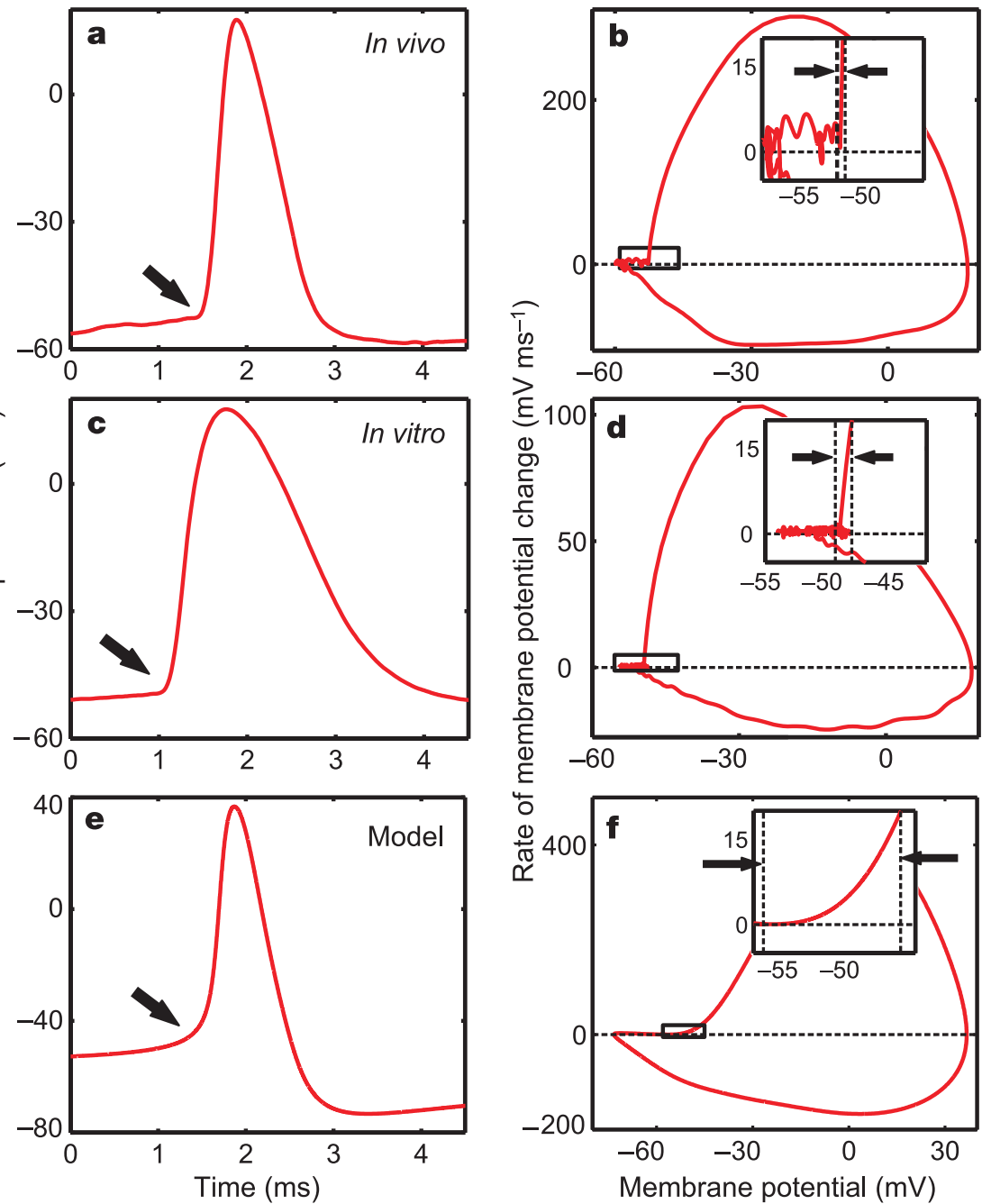

Fig 1. Rapid action potential onset in cortical neurons. The spikes recorded in vivo and in vitro have kinks at their initiation regime. In their phase plots, the voltage derivatives rise rapidly within a small voltage interval. For the conductance-based neuron model, the initiation part of the spike is smooth. The voltage derivative rises slowly in the phase plot. The figure is from 7 .

Some studies argued that the rapid AP onset seen at the soma might be an artifact of back-propagation of spiking voltage generated in the axon [8]. In subsequent work [9], rapid AP onset was found to have further implications for information encoding. In [9], AP onset rapidness was reduced in two ways shown in Fig 2. For the first method, the sodium concentration was reduced outside the neuron, such that there is less sodium current in each sodium channel. For the second method, TTX was used 
to block some of the sodium channels. In both conditions, the sodium current for the AP initiation was reduced which impaired the AP onset rapidness. Fig $2 \mathrm{~B}$ and $\mathrm{C}$ compare the encoding abilities of the neurons with and without the rapid AP onset. The encoding abilities in high frequency regime were impaired when the AP onset rapidness was reduced.

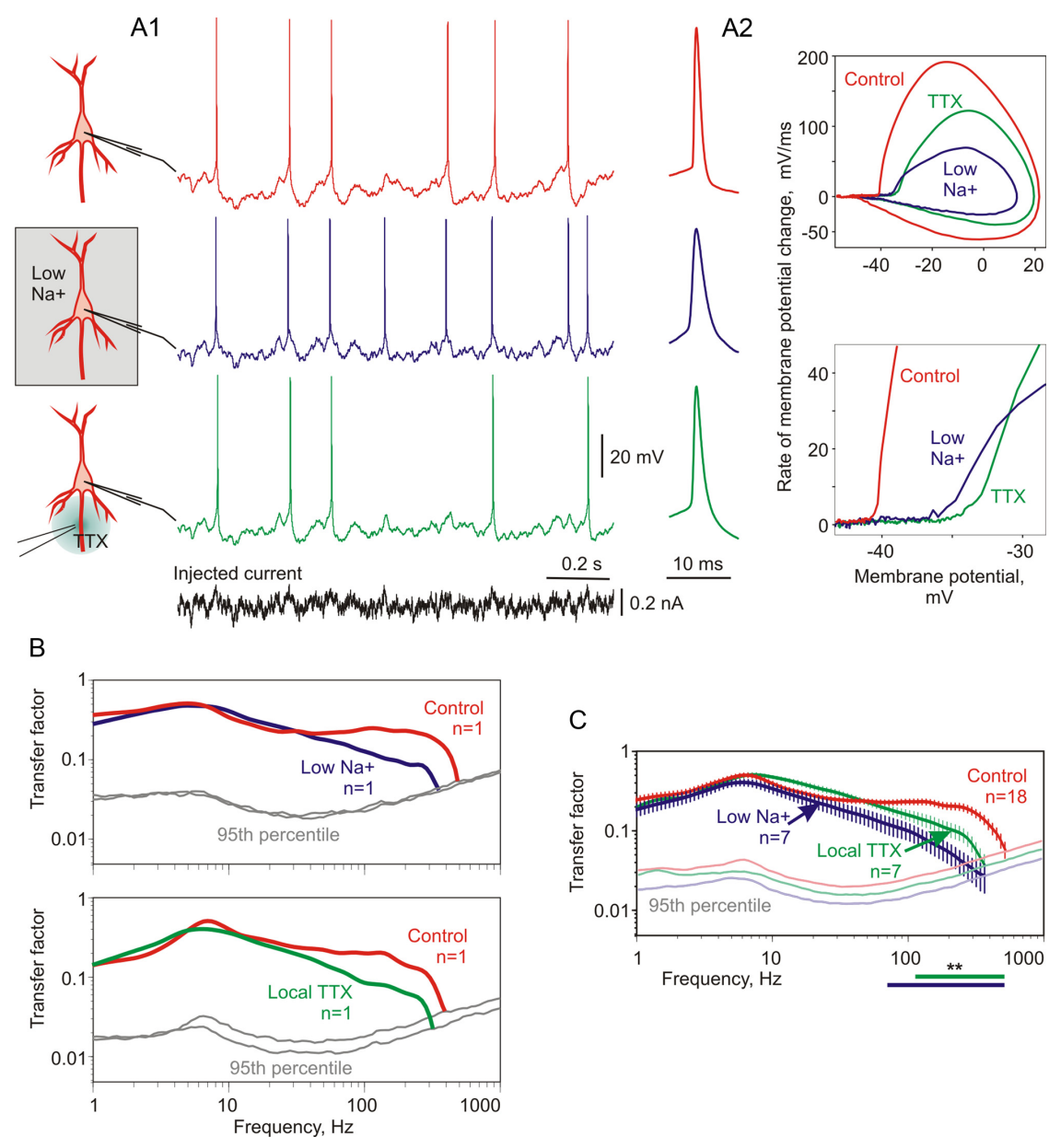

Fig 2. Reducing AP onset rapidness impairs the encoding ability of cortical neurons. A1. Reducing the sodium density outside of the neuron or blocking some of the sodium channels make the kink at the initiation of a spike less significant. A2. From the view of a phase plot, the AP generation dynamics are less rapid in the two cases examined in A1. B, C. Comparing the linear response functions of these two conditions with that of the intact neuron, the encoding abilities in high frequency regime are impaired. The figure is from [9].

Up till now, I explained the action potential generation dynamics of conductance-based models and highlighted an important property of the spikes in cortical neurons missing in some conductance-based models. In the next section, I will summarize experimental evidences on fast information processing in the brain. Then I will introduce the proposal of realizing fast information processing by population firing rate encoding. Following this path, I will introduce the experimental evidence on fast population encoding and the theoretical approaches examining the impact of neuron morphology 
and biophysics on the encoding ability. Based on the theoretical studies, I will pose the my specific scientific questions addressed in this thesis.

\subsection{Fast Information Processing in the Brain}

The brain is capable of fast information processing. If someone throws an apple towards another, it is quite simple for the receiver to read the trajectory of the apple, predict the upcoming position, and catch the apple. The whole process can take no more than a fraction of a second. In our daily lives, fast information processing is applied everywhere. It requires a quick reaction to stop the car to avoid an accident of hitting an unexpected pedestrian. In video games, a sequence of rapid and precise controls of the figure might be essential to realize perfect dodge and jump in one stage.

In an early experimental study [10, it was found that human brains can perform a highly demanding visual processing task in less than $150 \mathrm{~ms}$. In this experiment, the subjects were told to report whether a shortly given image contained an animal or not. The presentation time of one image was only $20 \mathrm{~ms}$. The types of animal images were versatile. What is more, the animals were pictured in their natural environments which could distract the subject. The subjects were asked to perform a go/no-go categorization. We can see that the whole task contains several stages of information processing in the brain. Reading the image requires visual information processing. Telling whether an animal exists or not requires recognition and decision making. Performing the final go/no-go categorization requires motor controls planned ahead. According to the experimental results, the subjects finished the task with an extremely high correct percentage in around $500 \mathrm{~ms}$. Using event-related potential recordings, the potential divergence of the no-go response from the go response indicated that the time it took for the visual processing stage was roughly $150 \mathrm{~ms}$ as shown in Fig 3 .

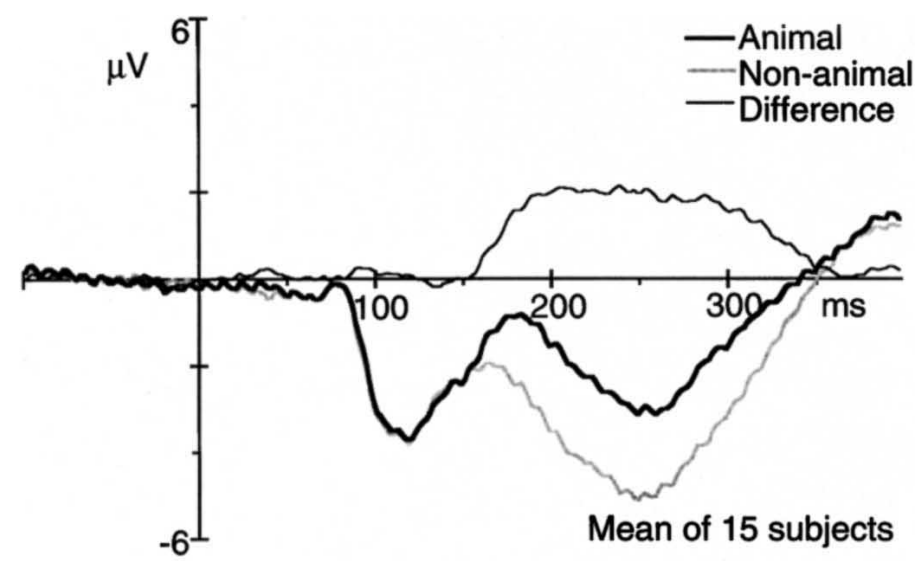

Fig 3. The averaged event-related potentials over 15 subjects. The event-related potentials diverge after $150 \mathrm{~ms}$. It is proposed to correspond with the decision-related activation after the visual processing of existence of animals. The figure is adapted from 10 .

The experiment above was based on complicated natural images. In a more simplified task, monkeys were trained to make a saccade towards to the target that may show up in different positions [11. The saccade reaction time was well below $300 \mathrm{~ms}$. Introducing a small period of darkness 
before appearance of the saccade target reduces the saccade reaction time to around $100 \mathrm{~ms}$. A more recent work [12] used the compelled-response paradigm to determine the time it takes for a monkey to make an accurate color discrimination. In the two-alternative choice task, the go signal was given before the appearance of two possible choices. The time gap separates the motor-planning stage from the decision-making stage. The experimental result shows that the color discrimination was performed in less than $30 \mathrm{~ms}$.

Following 10, the underlying mechanisms for fast information processing have been investigated 13.14 . Arguing that the behavioral responses during information processing are too fast to be encoded by the firing rates of individual neurons, which require time average over a longer time window, the authors proposed that information was encoded in the relative timing of a few first spikes in individual neurons. The firing rates in a larger time scale was proposed to describe the quantities of the stimulus rather than its identity. Recording from the afferents in the median nerve of the upper arm, the first few spikes were found to tell the directions of forces and the shapes of stimuli on the fingertips 13$]$.

An alternative idea may circumvent the problem of obtaining firing rate over a long time window. Instead of calculating the firing rates of individual neurons through long time averaging, the population firing rate of a large population of neurons can be obtained in a short time scale by population averaging. The information on the time varying stimulus could be coded through the population firing rate. In the next section, I will give an introduction to the findings on the evidences of fast information encoding with population firing rates.

\subsection{Fast Population Encoding}

In this section, I will first introduce the scientific intuition behind the proposal of population firing rate encoding. First, I will explain why researchers favor firing rate rather than firing pattern for the information encoding in the cortex. Second, I will introduce the experimental evidence on fast population encoding. Applying different stimulus types, several experiments have shown that a population of cortical neurons can represent the stimuli extremely well, which is far beyond the limitations on the firing rate of individual neurons or the membrane time constants of cortical neurons. Third, I will introduce an important property of population encoding, which will be used as a criterion for evaluating the performances of theoretical models in realizing fast population encoding.

\subsubsection{Fluctuation-Driven Firing and Weakly Correlated Corti- cal Neurons}

Cortical neurons display highly irregular firing patterns. Recordings from primary visual cortex and extrastriate cortex showed that the coefficients of variation $(\mathrm{CV})$ of the inter-spike intervals (ISI) are close to 1 , which indicates that the firing patterns mimic a Poisson-like random processes [15]. The distribution of ISI has an exponential decay. Further experimental studies showed that the higher order statistics of the ISI, the skewness coefficient, is inconsistent with that generated by a leaky integrate and fire model with Ornstein-Uhlenbeck Process stimuli [16. What is more, the irregular firing patterns are not reliable in different stimulus trails. Several experiments showed that the responses of cortical neurons to the same 
stimulus are highly variable 1720$]$. So it is unlikely that information is encoded in such stochastic and unreliable firing patterns.

In subsequent theoretical studies 21, 22, the origin of irregular firing was investigated. Each cortical neuron receives a large amount of synaptic inputs in a diverse range. The majority of synaptic inputs are excitatory. The impact of an individual synaptic input is quite limited to move the voltage towards or away from the spiking threshold. It is speculated that irregular firing is originated from highly fluctuating synaptic inputs. However, one previous study showed that with irregular excitatory synaptic inputs, an integrate and fire model failed to realize irregular firing [15], which seemed to provide contradictory evidence on this opinion. In the works of Shadlen 21, 22], as shown in Fig 4, they reexamined this model setup with a different approach. First, they reproduced the regular firing pattern found in [15]. Then they examined the hypothesis that irregular firing is a consequence of coincidence detection of afferent inputs. In this case, to realize irregular firing, the time constant of the post synaptic potential decay was tuned to $1 \mathrm{~ms}$, which was much smaller than the parameter range of cortical neurons. In the third case, they took the inhibitory inputs into consideration to balance the excitatory inputs. With a reasonable time constant parameter, the neuron model acted as an integrator of synaptic inputs. The spikes were a result of fluctuating synaptic inputs. Highly irregular firing was reproduced in this case.

Another observation from the theoretical studies above is that the neuron's voltage is fluctuating in the subthreshold region when acting as an integrator than that acting as an coincidence detector. This observation is also supported by experiments. Several experimental studies show that with intense synaptic bombardment, the membrane voltage is highly fluctuating below the spiking threshold [23 25]. In visual cortex [23], the fluctuation amplitude increases with the contrast of optimally oriented gratings.

Up till now, I have introduced the firing properties of cortical neurons, from which we can see that driven by large numbers of excitatory and inhibitory synaptic inputs, the membrane voltage mainly fluctuates below the spiking threshold. The firing pattern generated under such stochastic inputs are highly irregular. In the second part of this section, I will discuss correlations between neighboring cortical neurons. We will see that cortical neurons are weakly correlated. Based on this feature, the idea of encoding information through population firing rates of weakly correlated cortical neurons will be discussed.

Several experimental studies focused on the question how correlated the signal and the noise carried by a population of cortical neurons are 2630 . If the signal carried by cortical neurons are highly correlated, then there is a redundancy on the information transmission. On the other hand, if the noise carried by the cortical neuron are highly correlated, then it would be difficult to average out the noise by receiving a large number of synaptic inputs. Paired neuron recordings with single microelectrode on inferior temporal cortical neurons showed that the signal carried by adjacent neurons are relatively heterogeneous 26. The noises in these neurons are much more uncorrelated than the signal. The spike count covariation in middle temporal visual area were found to be relatively weak 27]. The averaged correlation coefficient is 0.12 . Neuron pairs whose preferred directions are less than $90^{\circ}$ have more positive correlations compared to those whose preferred directions are more than $90^{\circ}$.

The results above indicate that neighboring neurons with similar tuning 
A

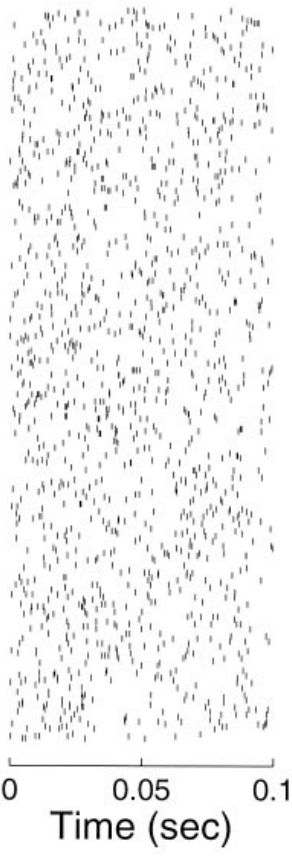

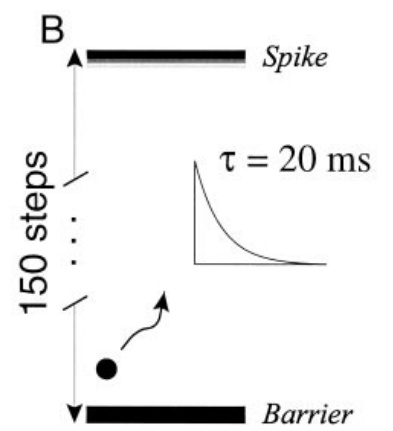
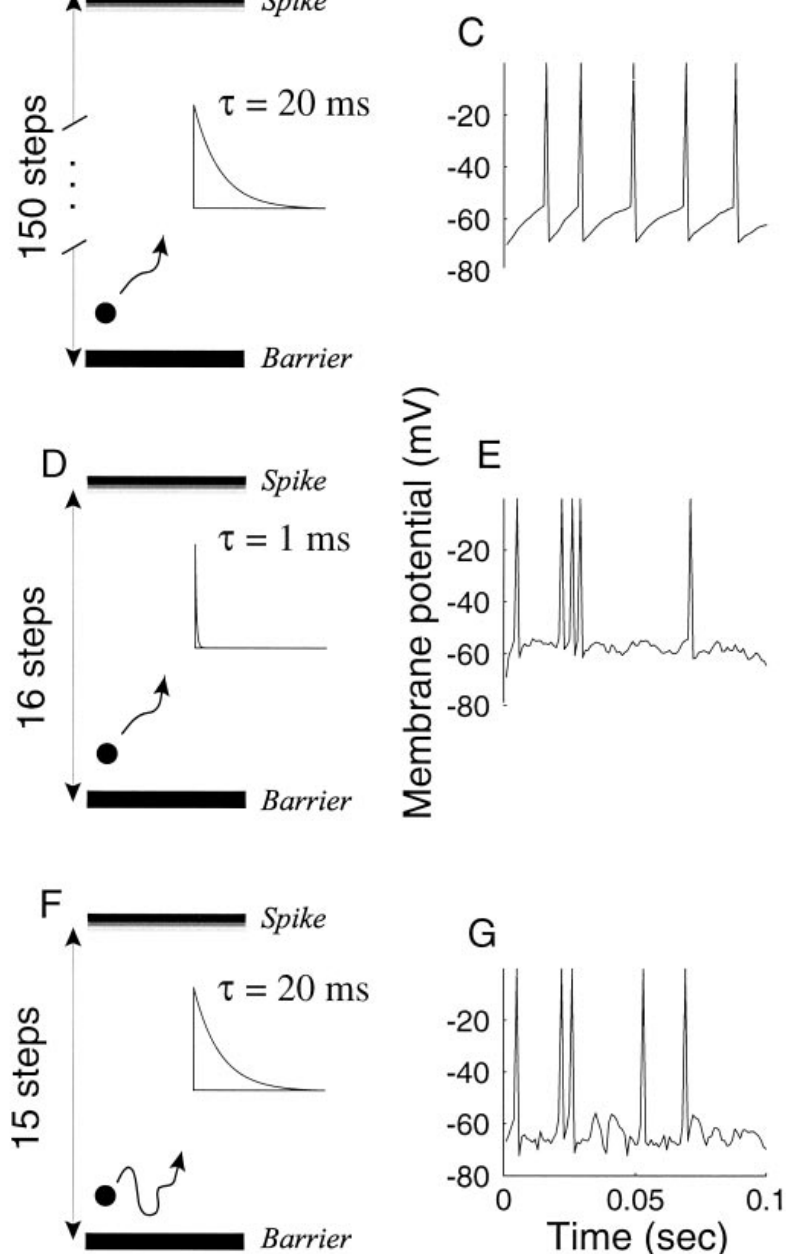

Fig 4. Three models on the origin of irregular firing in cortical neurons. A. 300 excitatory irregular synaptic inputs injected into the integrate and fire model. B, C: Regular firing pattern is obtained when the time constant of the post synaptic potential decay in the model is set to 20ms. D, E: Irregular firing pattern is obtained when time constant of the model is set to 1ms. F, G: irregular firing pattern is obtain when 300 inhibitory inputs are introduced to balance the excitatory inputs. The time constant of the model is $20 \mathrm{~ms}$. The figure is from 22 .

share similar signals and noises. In a subsequent study, with multi-electrode recording, it investigated the impact of distances on signal correlations and noise correlations of pairs of motor cortex neurons and parietal cortex neurons [28]. As shown in Fig 5, when two neurons are close to each other, a larger signal correlation usually implies a larger noise correlation. The scatter points between the two have a more positive slope in the linear fit. Increasing the distance between two neurons makes the positive relation diminishes. The noise correlations are much weaker than the signal correlations. Also, the averaged noise correlation over all neuron pairs is close to zero. Similar conclusions are drawn in a following study 30 on prefrontal cortex. This work showed that the correlation between excitatory pyramidal neurons which transmit information from one area to another, are 
less than those of inhibitory neurons which have more local effects. A more recent work 31], with more refined examination of the noise correlation of visual cortex, reported a much smaller correlation than previous results, and proposed that either the shared inputs are limited or the neurons actively decorrelated. A theoretical work demonstrated a recurrent neural network model reproduced the weak correlation property 32$]$.

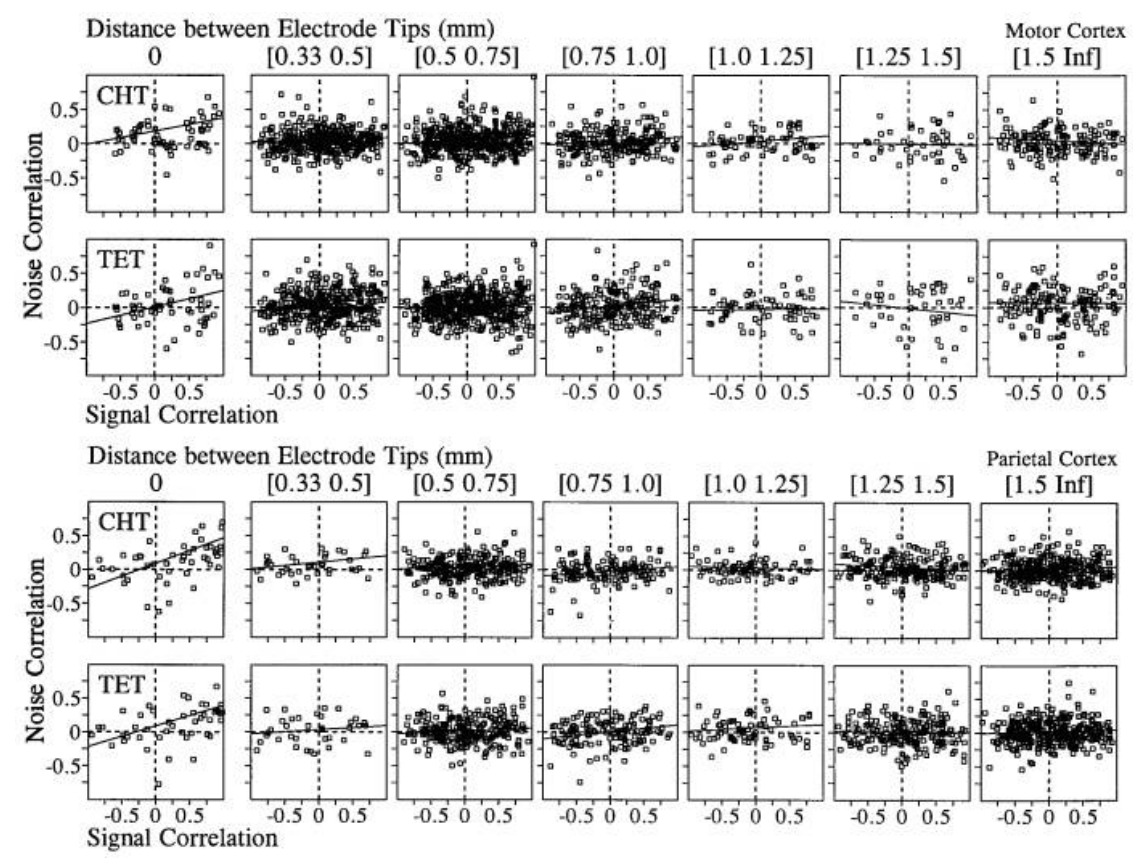

Fig 5. The relation between signal correlation and noise correlation in pairs of motor cortex neurons and parietal cortex neurons at different distances. CHT represents the center hold time before the presence of peripheral targets. TET represents the total experimental time between the target onset and the end of the movement. In motor cortex, when a pair of neurons are close to each other, we observe a more positive association between signal correlations and noise correlations. The fitted curves of scattered points have larger slopes when two neurons are close to each other. The noise correlations are less distributed than the signal correlations in all cases. Similar results are observed in parietal cortex. The figure is adapted from [28].

With the knowledge that cortical neurons are working in the fluctuationdriven regime and firing highly irregular spikes, and that a population of cortical neurons are weakly correlated, the following conclusions can be drawn. Assume that a common signal is injected into a population of weakly correlated cortical neurons. The signal amplitude is much smaller than the background noise, such that the firing patterns of individual neurons are dominated by stochastic fluctuations. It might be difficult to interpret the underlying signal from the irregular firing of one neuron. However, for a large population of weakly correlated neurons, each neuron's background noise can be regarded independent of the others'. The hidden properties of the common input can thus be reflected in the population firing rate. This is the idea of population encoding. 


\subsubsection{High Bandwidth Encoding}

In this section, I will summarize experimental works investigating the population encoding of cortical neurons. I will show that the cortical neurons can represent the small signals merged in the background noise unexpectedly well. The encoding ability is described by the linear response function, which displays a high bandwidth encoding for cortical neurons.

Several experimental studies have shown that a population of cortical neurons are capable of representing a small signal merged in background noise with their population firing rate extremely well [9, 33 36]. In these experiments, the cortical neurons are assumed to be weakly correlated, such that the collective behavior of a whole population can be approximated by the recordings on a few neurons over multiple trails. In each trail, the signal is the same, but the background noise are independent of those in the other trails. Two typical types of signals are step functions and sinusoidal functions.

For layer $2 / 3$ pyramidal neurons 9,36 , when responding to a small step current merged in the background fluctuations, the cortical neurons could detect the signal instantaneously. Within 1 to $2 \mathrm{~ms}$, the population firing rate exhibited a drastic change once the step current was added to the background fluctuations [9, 36. As shown in Fig 6, the small step current was chosen to have an amplitude of unitary postsynaptic current, which is much smaller than the noise fluctuations shown as the blue curve. The contribution of the step current is negligible seen from the combined stimulus shown as the black curve. If injected into a cortical neuron, no spikes can be generated. Only small amplitude voltage fluctuations are observed. If we inject the cortical neurons with the combined stimulus, highly irregular firing pattern are obtained, which give no clue about the underlying small step current. However, if we examine the population firing rate, a strong and instantaneous change follows the increase of the step current. Similarly, the population firing rate drops instantaneously with the decrease of the step current. The results above give a clear and intuitive evidence on the signal tracking ability of a population of cortical neurons.

The fast signal tracking by a population of cortical neurons can be represented in another way. Adding a small sinusoidal signal in the background noise, a population of cortical neurons can represent the signal with a sinusoidal population firing rate having the same frequency $9,33,36$. As shown in Fig 7 , the red curves are the sinusoidal signals. The black curves are the total inputs composed of the stochastic stimulus and the sinusoidal signals. Comparing the left and right panels, we can see that depending on the frequency of the sinusoidal signal, the population firing rate has the same fluctuation frequency but a different phase shift from the signal.

In the linear regime, the population firing rate fluctuations are proportional to the small amplitudes of sinusoidal signals. The ratio of the population firing fluctuation amplitude to the sinusoidal fluctuation amplitude is the dynamic gain of cortical neurons at related frequency. Experimentally, it has been found that the dynamic gain of cortical neurons can be maintained up to a few hundreds $\mathrm{Hz} 9,33$ 36. This implies that cortical neurons can encode high frequency signals almost as good as low frequency signals. The encoding ability is not limited by the membrane time constant and the firing rate of individual neurons. We call this property of high bandwidth encoding also ultrafast population encoding. Ultrafast population encoding is not limited to layer $2 / 3$ pyramidal neurons in the visual cortex 9,36 . It 


\section{Single neuron}

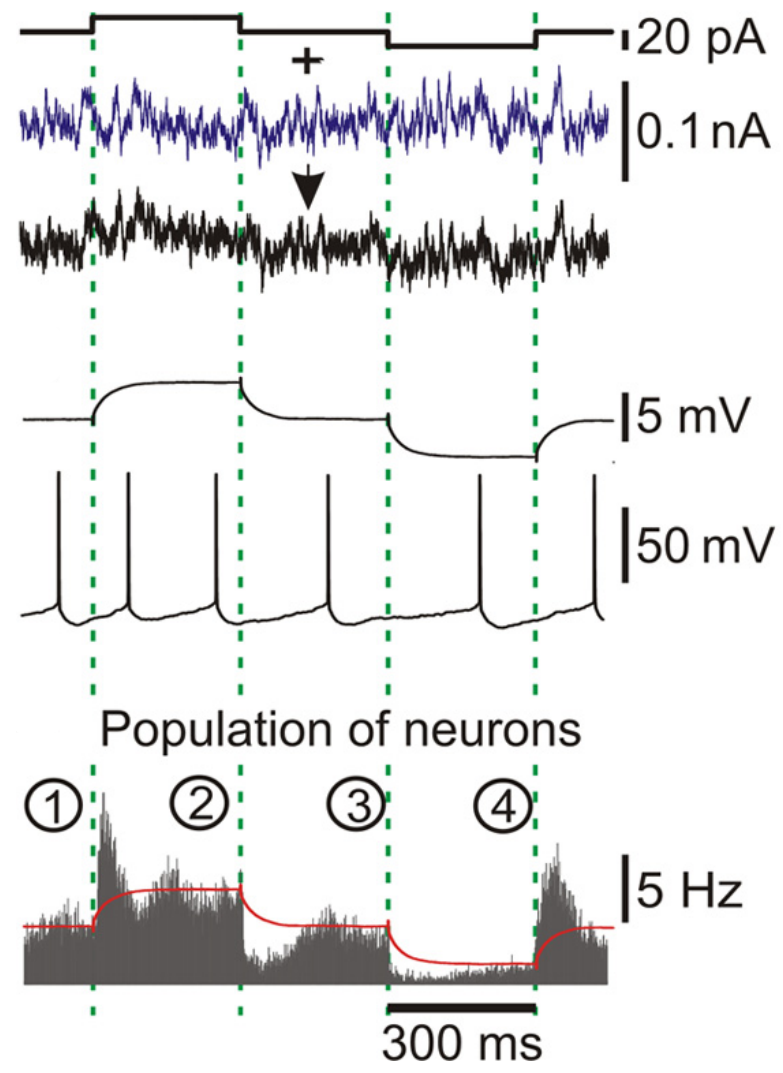

Fig 6. Fast population encoding in response to a small step current merged in background noise. A small step current is added to a much larger stochastic noise. The step current alone is not able to trigger spikes. The spike pattern generated with the combined stimulus is highly irregular. Seen from the population firing rate, an instantaneous change with 1 to $2 \mathrm{~ms}$ follows with the step current change. The figure is adapted from 36 .

is also found in layer 5 pyramidal neurons in the motor and somatosensory cortex 3335 .

To quantify population encoding ability, the linear response function of the population firing rate has been adopted in experimental cell physiology. The linear response function represents the dynamic gain as a function of frequency. This concept was first introduced by Knight [37] and later theoretically elaborated in more biologically realistic and detailed neuron models 38 47. Theoretical progresses is summarized in later sections. Here I will first discuss the linear response curves recorded in experiments. The work of 33 is one of the first studies that measured the linear response curves of cortical neurons. This work implemented two types of stochastic stimuli named as subthreshold stimulus and suprathreshold stimulus as shown in Fig 8 . The subthreshold stimulus had large fluctuations and a small mean, of which the mean itself was not able to trigger spikes. 

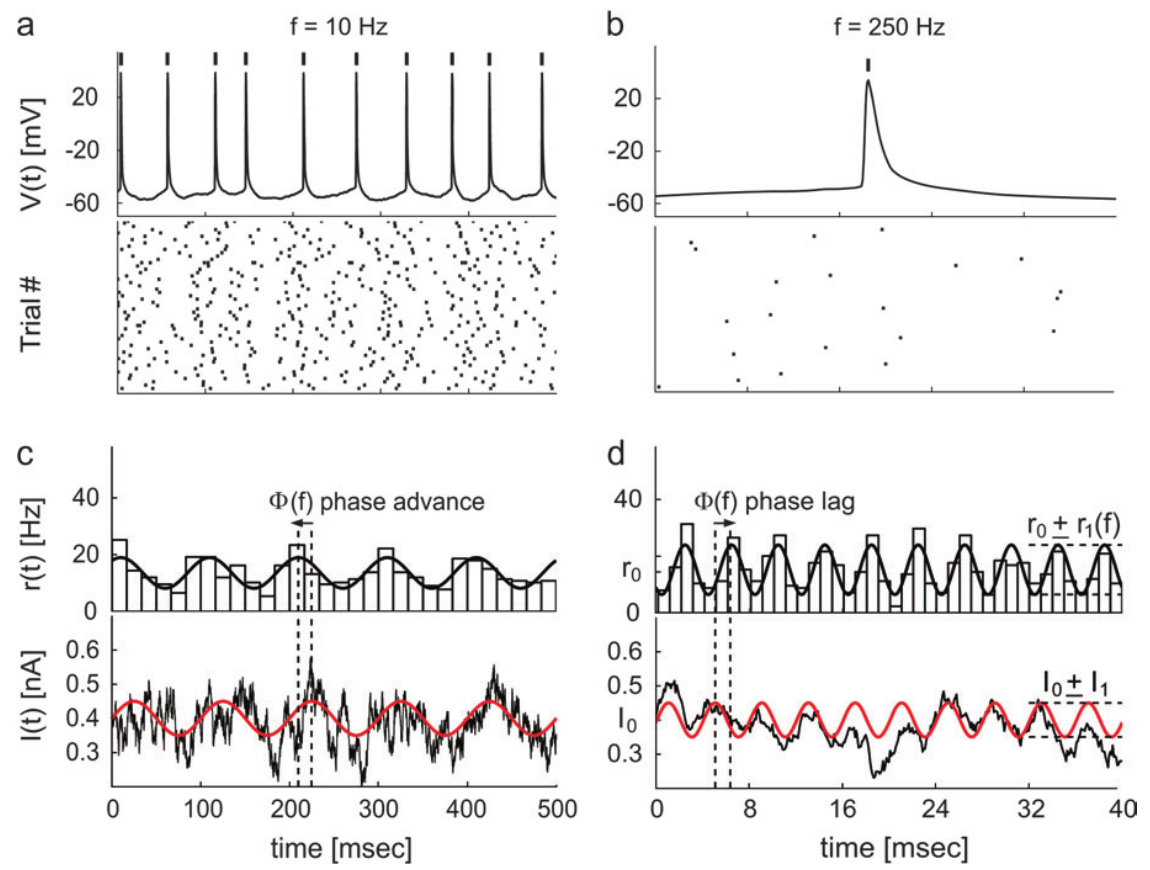

Fig 7. Fast population encoding of a small sinusoidal current merged in background noise. A sinusoidal signal is added to the background noise shown in the bottom figures. The red curve is the signal, and the black curve is the total input. In the left panel, the frequency of the sinusoidal signal is $10 \mathrm{~Hz}$. In the right panel, it is $250 \mathrm{~Hz}$. The upper part of the panel is the spiking voltage trace and the raster plot of the spike times over different trails. The lower panel is the sinusoidal population firing rate fluctuations. The figure is adapted from 33 .

The suprathreshold had small fluctuations and a large mean, of which the mean itself was able to generate spikes. In both cases, the dynamic gain maintained high values up to a few hundred Hz. In the suprathreshold case, the linear response curve had a strong resonance at high frequencies. The phase shift curves are similar in two conditions, and do not show a saturation in the high frequency limit.

\subsubsection{Sensitivity of the High Bandwidth Encoding to the Cor- relation Time of Background Fluctuations}

One characteristic property of ultrafast population encoding in cortical neurons is the sensitivity of the dynamic gain to the correlation time of background current fluctuations. Increasing the correlation time of stochastic inputs enhances the dynamic gain at high frequency. This phenomenon was first predicted in theoretical work on leaky integrate-andfire model (LIF) 39 shown in Fig 9 This study shows theoretically that injecting a white noise into the LIF model, the linear response function decays with a slope of $-\frac{1}{2}$ in the log-log scale. The phase shift converges to $45^{\circ}$ lagged behind the input. It also shows that when the correlation time of input becomes a non-zero number, the linear response function of the LIF model will converge to a finite value in the large frequency limit. The lag in the phase shift is also eliminated in high frequency limit. What is 


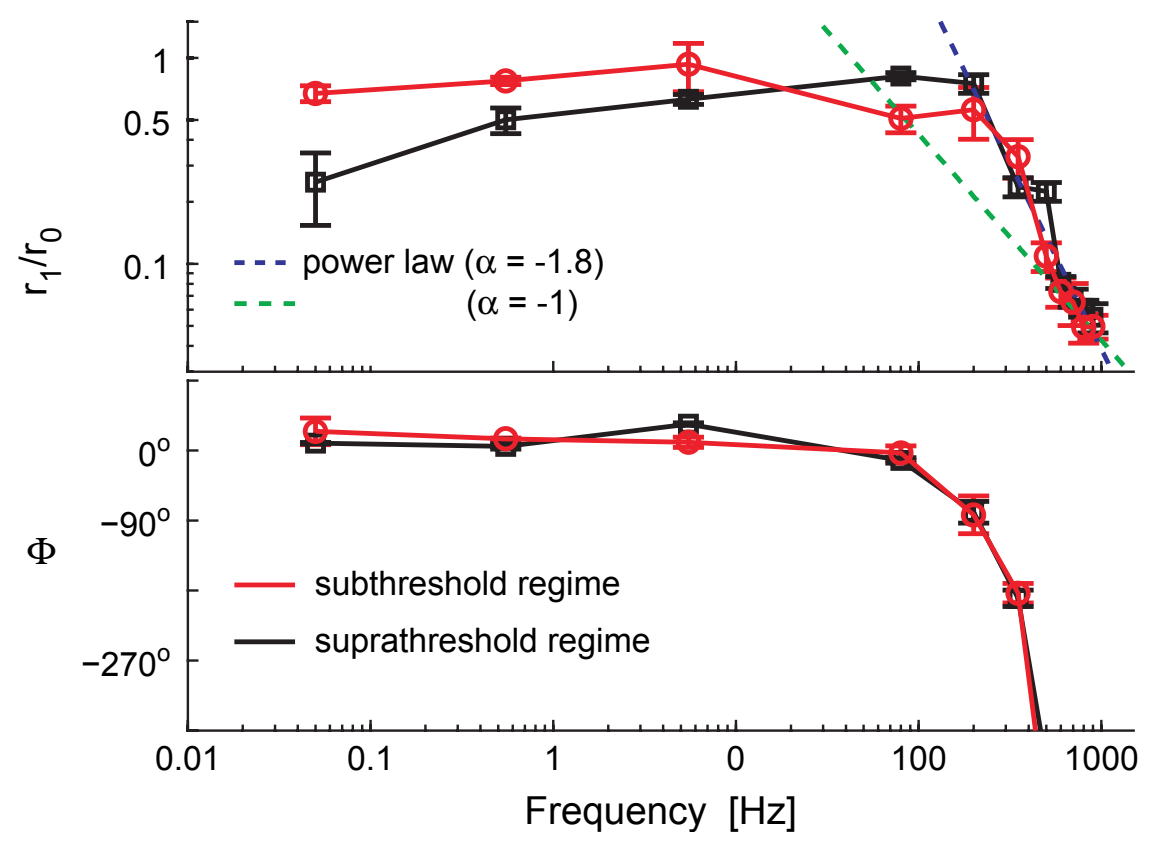

Fig 8. Linear response functions of layer 5 pyramidal neurons. The red curve represents the linear response curve generated with a stochastic input having a large noise fluctuation. The mean of the input is not able to trigger spikes. The black curve represents the linear response curve generated with a stochastic input having a weak noise fluctuation. The mean of the input is able to trigger spikes. In both conditions, the cortical neurons realize high bandwidth encoding above $100 \mathrm{~Hz}$. The lower panel shows the phase shift for different frequencies in these two conditions. The figure is adapted from 33 .

more, a larger correlation time of inputs leads to a larger asymptotic value of the dynamic gain.

Later the sensitivity of the dynamic gain to the correlation time of the input was also observed in experimental studies 36$]$ shown in Fig 10 . In vitro recordings of layer $2 / 3$ neurons show that the dynamic gain in high frequency regime is enhanced when increasing the correlation time of background fluctuations. This experimental study also provides supporting evidence that ultrafast population encoding exists in vivo, rather than a phenomenon observed in vitro.

\subsection{Theoretical Approaches on Population Encoding}

In this section, I will summarize theoretical approaches to population encoding. First, I will highlight questions that exists in theoretical modeling. Highly simplified models can realize high bandwidth encoding. However, many conductance-based models with biophysical plausible AP generation mechanisms fail to do so. Second, I will introduce a theoretical approach to understand the impact of AP onset rapidness on population encoding. Several lines of modeling work have shown that the population encoding ability is enhanced when the AP onset dynamics becomes more rapid. Following this finding, several lines of study have emerged to explore how neuron morphology and AP generation mechanisms shape high bandwidth 

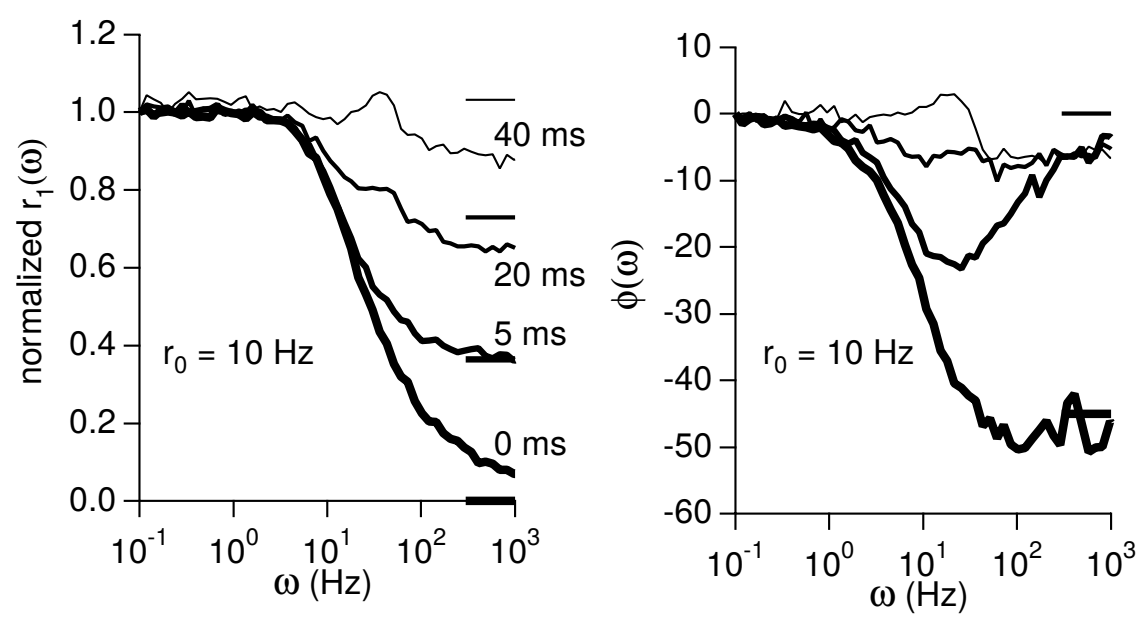

Fig 9. Sensitivity of the dynamic gain to the correlation time of the stimulus in theoretical modeling. The mean firing rate of the model is $10 \mathrm{~Hz}$. Increasing the correlation time of inputs enhances the encoding ability in high frequency regime. The phase lag is eliminated more quickly for a larger correlation time. The figure is adapted from [39].
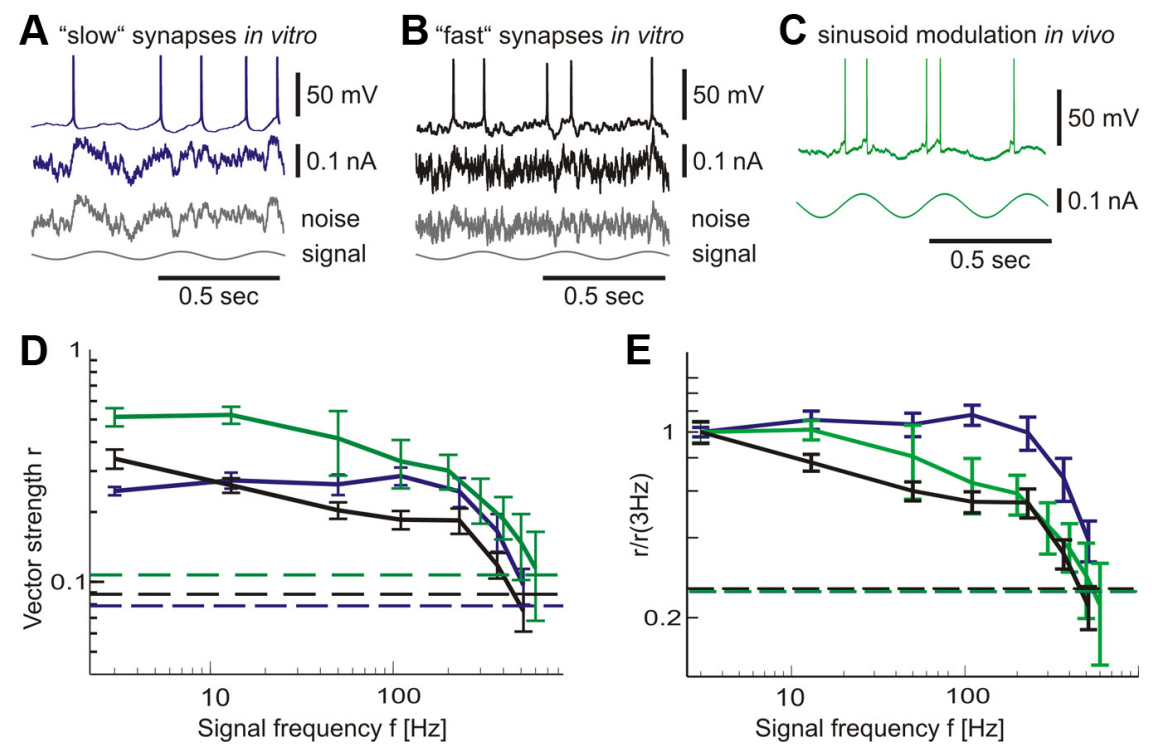

Fig 10. Sensitivity of dynamic gain to the correlation time of the stimulus in experiments. A, B: sinusoidal signals are added to the background noise with different correlation times. The correlation times in $\mathrm{A}, \mathrm{B}$ are $50 \mathrm{~ms}$ and $5 \mathrm{~ms}$ separately. The recordings are in vitro. D, E: the linear response curve has a higher bandwidth for larger correlation time. C. For comparison, a sinusoidal signal is injected in vivo. It shows that cortical neurons in vivo can also encode high frequency signals. The dash lines below the linear response curves are the 95 per cent significance level. The figure is adapted from 36].

encoding. In the last part of this section, I will summarize two analytical methods to calculate linear response functions. 


\subsubsection{Ultrafast Population Encoding and Realistic Neuron Mod- eling}

Neuron models range from the simple LIF model to complicated multicompartment models. This simple LIF model acts as an integrator of all afferent inputs. Spike generation dynamics are not considered in this model. To generate spikes, a hard threshold is set. A spike is generated once the voltage reaches the threshold. There is also no voltage downward regulation mechanism included. The voltage is reset by hand after a spike is generated. For more complicated quadratic integrate and fire neuron model, spike generation dynamics is added to the model [43. The spiking voltage will go to infinity in finite time, and then rise from minus infinity again. In this sense, no specific spiking threshold and reset voltage are required. Another model which also shares the property of spiking voltage going to infinity in finite time is the exponential integrate and fire model [41. In this model, the spike generation dynamics are described by an exponential function, which is an approximation to the sodium activation in a conductance-based model [6]. The most well-known model that provide a complete spike generation dynamics with ion channels is the Hodgkin-Huxley model [5. In this conductance-based model, the sodium current is the main driving force for generating spikes. When the voltage is high enough, the inactivation of the sodium channels will block the sodium current. Then the potassium current will bring down the voltage to finish a spike. Beyond the Hodgkin-Huxley model, the detailed structure of the neuron morphology are considered into the model setup. Instead of simplifying the neuron as a single compartment soma, an axon and a dendrite are added to the neuron models 48, 49]. Different ion channels may also be distributed in different neuron compartments rather than concentrated at one point.

Increasing computation power allows us to describe the neuron model to an extent of unprecedented complication. However, one may wonder whether the most complicated model is the most realistic model. As it is point out in 50, a realistic model should be capable of reproducing the key functions of the neurons we are trying to simulate. If we take the ultrafast population encoding as the key feature of a cortical neuron, then the simplest LIF model is a quite elegant and "realistic" model. Not only can it realize high bandwidth encoding, but also the encoding ability in the high frequency regime is sensitive to the correlation time of the input as shown in Fig 9. In comparison, the complicated conductance-based model can be quite deficient in capturing the encoding properties of cortical neuron. Many linear response functions of conductance-based models are found to have cutoff frequencies on the scale of the firing rates. Also, the dynamic gain in the high frequency regime is insensitive to the correlation time of inputs [41,46]. The exponential integrate and fire model also displays similar behavior when its parameters are tuned to match a conductancebased model [41]. The results above indicate that single-compartment conductance based models may have some key functional features missing. Some studies resort to the more complicated multi-compartment model framework $48,49,51,52$. While some others focus on the AP generation dynamics [46,53]. A more detailed introduction on these two parts will be given in following sections. Here as the next step, I will first review an AP generation phenomenon that has been proposed closely associated with ultrafast population encoding, and related theoretical modeling works on 
this phenomenon.

\subsubsection{The Impact of AP Onset Rapidness on Population Encod- ing}

In the previous section, I have introduced the studies on AP generation dynamics. One important phenomenon that is observed in cortical neurons but missing in conductance-based neuron models is the rapid AP onset 7. When generating an AP, the neuron voltage will rise rapidly from subthreshold fluctuations. As shown in the phase plot, there is a kink at the initiation part of a spike. While for the conductance-based models, related regions in the phase plot are quite smooth as shown in Fig 1. Rapid AP onset is proposed to be closely associated with ultrafast population encoding. One experimental study showed that impairing the AP onset rapidness will reduce the encoding ability of cortical neurons 9 . The dynamic gain in the high frequency regime decays faster if we reduce the sodium current per sodium channel or block some of the sodium channels shown in Fig 2 .
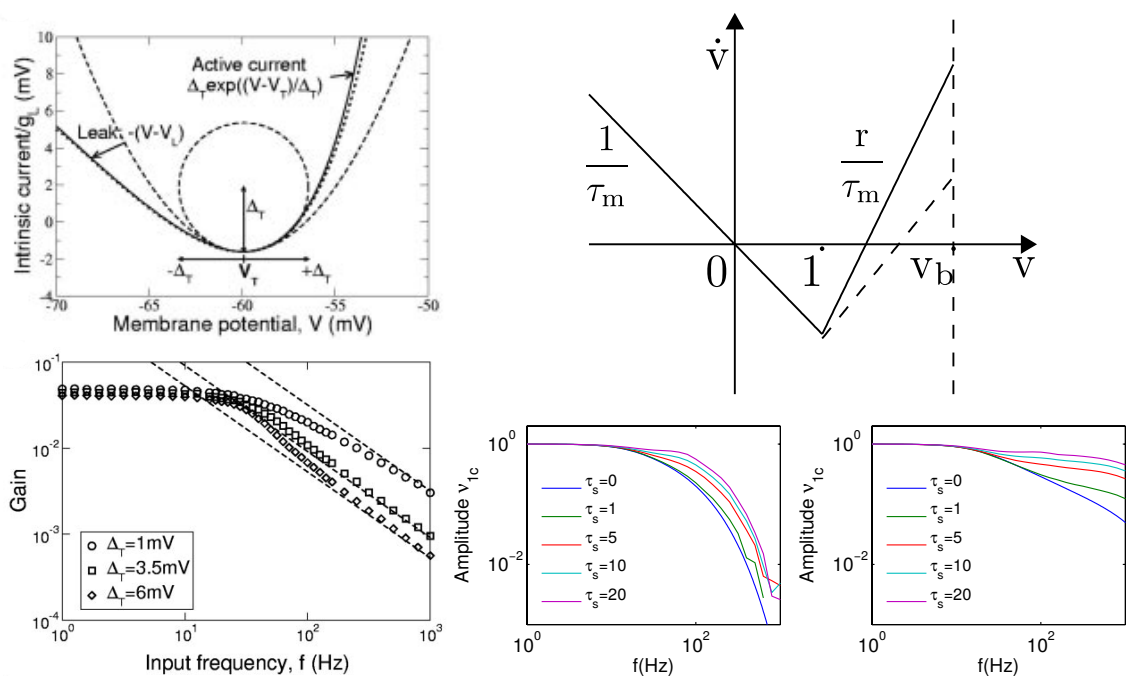

Fig 11. The impact of AP onset rapidness on population encoding. In the left column, $\Delta_{r}$ describes the radius of the curvature in the I-V curve. A smaller $\Delta_{r}$ indicates a faster AP onset. The high bandwidth encoding is enhanced for smaller $\Delta_{r}$ values. In the right column, the AP onset dynamics is described by a linear curve. The parameter $r$ defines the rapidness of AP onset. $r$ is 10 in the figure of bottom left. $r$ is 100 in the figure of bottom right. $\tau_{s}$ is the correlation time of input. A more rapid AP onset improves the encoding ability of the neuron model. The linear response function is more sensitive to the correlation time of the input. The figures are adapted from [41,45].

Several theoretical modeling works have shown that increasing the onset rapidness of AP generation can improve the encoding ability of the neuron models 41, 43, 45, 46. In the early study of 41, by decreasing the radius of the curvature in the I-V curve of EIF model, the spike generation voltage will rise more rapidly. The linear response functions have a better encoding in the high frequency regime shown in Fig 11. Similarly in a piece-wise linear model, the slope of the I-V curve represents the AP onset rapidness 45]. Increasing the slope will make the neuron model converge 
to a LIF model, which reproduces ultrafast population encoding [39]. The models above describe the onset rapidness with a functional parameter without explicitly representing it with biophysical parameters. In [46], it was proposed that cooperative gating of sodium channels could act as the underlying mechanisms of rapid onset. According to this proposal, activation of one sodium channel may increase the activation probability of neighboring sodium channels, which makes the collective sodium current activation dynamics more rapid than that of individual channels. With cooperative gating of sodium channels, AP onset is more rapid. Together, the encoding ability is enhanced.

With the knowledge of close connection between rapid AP onset and ultrafast population encoding, rapid AP onset has been added as a criterion to be examined in subsequent modeling works trying to reproduce ultrafast population encoding $488,49,52,53$. However, it is not clear whether these two properties are really equivalent to each other. In the later sections, we will take more detailed look at the underlying mechanisms of rapid AP onset and ultrafast population encoding in different hypotheses.

\subsubsection{Fokker-Planck Approach and Gauss-Rice Approach to Pop- ulation Encoding}

Before introducing the hypotheses on the underlying mechanisms of ultrafast population encoding, I will give a brief summary of two analytical methods for calculating the linear response function. Analytical methods are limited to some idealized conditions or may require additional assumptions, which makes them unsuitable tools when dealing with complicated models describing detailed biophysical properties of the cortical neurons. However, they still provide insight on many features of linear response functions.

The first analytical method is the Fokker-Planck approach. The introduction of this method follows the work of 39,45. In this case, the neuron model is driven by a stochastic input composed of a small amplitude signal and background noise. The simplest background noise that can be tackled analytically is white noise with a zero mean and constant variance. The small amplitude signal is a sinusoidal function of time. The voltage fluctuation dynamics can be described as a stochastic differential equation. As for the LIF model in [39, it has the following form:

$$
\tau_{m} \frac{d V}{d t}=V_{\text {rest }}-V+I_{\text {in }}+\eta(t)
$$

Here $\tau_{m}$ is the membrane time constant. $V$ is the neuron voltage. $V_{\text {rest }}$ is the resting potential. $I_{i n}$ is the small amplitude signal. $\eta(t)$ is the zero mean white noise, with an intensity of $\sigma^{2}$. The spike threshold is denoted $V_{t h}$. A spike is generated when the voltage reaches the spike threshold. The voltage is reset to $V_{\text {rest }}$ after a spike.

To calculate the linear response function based on the LIF model, we need the population firing rate $r(t) . r(t)$ can be decomposed as a mean firing rate $r_{0}$ determined by the background noise, a sinusoidal fluctuation term $r_{1}(t)$, and higher order nonlinear terms. The linear term $r_{1}(t)$ determines the dynamic gain and the phase shift.

For infinite numbers of LIF neurons driven by different realizations of the background noise together with the same sinusoidal signal, the voltage values at each moment obey a probability distribution denoted as $P(V, t)$. The Fokker-Planck approach is based on the equation for $P(V, t)$ as below: 


$$
\tau_{m} \frac{\partial P}{\partial t}=\frac{\sigma^{2}}{2} \frac{\partial^{2} P}{\partial V^{2}}+\frac{\partial}{\partial V}\left[V-I_{\text {in }}(t)-V_{\text {rest }}\right] P
$$

The population firing rate is associated with the probability flux at the spike threshold $V_{t h}$.

$$
r(t)=-\left.\frac{\sigma^{2}}{2 \tau_{m}} \frac{\partial P(V, t)}{\partial V}\right|_{V=V_{t h}}
$$

For the LIF model with white noise, the linear term $r_{1}(t)$ can be calculated analytically [39], so that we can get the linear response function explicitly. For a piece-wise linear model with a finite boundary, which is a generalization of the LIF, the linear response function can be derived analytically in the same way with further boundary conditions between different linear pieces [45]. The piece-wise linear model also analytically reveals how the linear response function decays in the limit of infinite threshold.

One limitation of the Fokker-Planck approach is that it can't provide explicit expressions of the linear response functions when the correlation time of the background noise is non-zero. However, with the Gauss-Rice approach, we can provide an estimation of the linear response function when the background noise has a non-zero correlation time. Below I will give a brief introduction to the Gauss-Rice approach. The neuron model is also the LIF model. The stochastic background noise is generated by an Ornstein-Uhlenbeck process represented as:

$$
\tau d I=-I d t+\sqrt{2 \tau} \sigma d W(t)
$$

$\tau$ is the correlation time of the noise. $\sigma$ is the standard deviation of the noise. $W$ is a Wiener process satisfying $W_{t+d t}-W_{t} \sim N(0, d t)$.

The Gauss-Rice approach has the following assumptions on the neuron dynamics [44, 47]. In the previous case, the neuron model has a spiking threshold across which the voltage will be reset. The voltage distribution has a cutoff at the threshold. In the Gauss-Rice approach, a spike is generated when the voltage crosses the threshold from below. The voltage will not be reset but keep fluctuating after the spike time as shown in Fig 12. We assume the voltage distribution and the voltage derivative distribution are Gaussian. Following [47], when the voltage variance is stationary, the voltage derivative is uncorrelated with the voltage. So the probability density at time $t$ can be represented as:

$$
p(V, \dot{V} \mid t)=\frac{1}{2 \pi \sigma_{V} \sigma_{\dot{V}}} e^{-\frac{(V-\bar{V}(t))^{2}}{2 \sigma_{V}^{2}}} e^{-\frac{(\dot{V}-\bar{V}(t))^{2}}{2 \sigma_{\dot{V}}^{2}}}
$$

Here $\sigma_{V}$ and $\sigma_{\dot{V}}$ are the standard deviations of the voltage and voltage derivative. $\bar{V}(t)$ and $\bar{V}(t)$ are the mean voltage and mean voltage derivative at time $t$. The population firing rate is the rate crossing the threshold $\theta$ from below.

$$
\begin{aligned}
\nu(t) & =\langle\delta(V-\theta) \Theta(\dot{V}) \dot{V}\rangle_{V, \dot{V}} \\
& =\left.\int_{0}^{\infty} \dot{V} p(V, \dot{V} \mid t)\right|_{V=\theta} d \dot{V}
\end{aligned}
$$


Linearizing the firing rate to the first order, we have:

$$
\nu(t) \approx \nu_{0}\left(1+\frac{\theta}{\sigma_{V}} \cdot \frac{\bar{V}(t)}{\sigma_{V}}+\sqrt{\frac{\pi}{2}} \cdot \frac{\overline{\dot{V}}(t)}{\sigma_{\dot{V}}}\right)
$$

Fourier transforming both sides of the equation, we can obtain the dynamic gain as a function of frequency:

$$
\nu_{1}(\omega)=\nu_{0}\left(\frac{\theta}{\sigma_{V}^{2}}+i \omega \sqrt{\frac{\pi}{2}} \cdot \frac{1}{\sigma_{\dot{V}}}\right) \bar{V}(\omega)
$$

where $\bar{V}(\omega)$ can be obtained with the Fourier transform of the LIF model equation.

$$
i \omega \tau_{m} \bar{V}(\omega)=-\bar{V}(\omega)+\bar{I}(\omega)
$$

Here $\bar{I}(\omega)$ is the Fourier transform of the mean of the input. The stochastic background noise is averaged out, so here it is the Fourier transform of the small amplitude sinusoidal signal. In [44], it was shown that both $\sigma_{V}$ and $\sigma_{\dot{V}}$ can be obtained analytically. In this sense, we can derive the linear response function analytically.

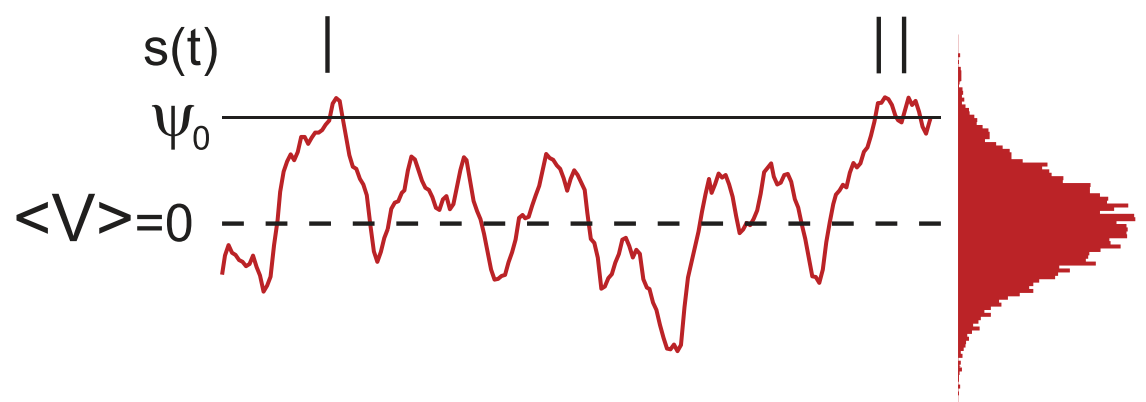

Fig 12. Spike generation mechanism of Gauss-Rice approach. In this figure, the mean voltage is assumed to be $0 . \Psi_{0}$ is the spike threshold. Each time the voltage crosses the threshold in the positive direction is counted as a spike. The voltage will not be reset after the spike generation. $s(t)$ is the spike time represented as the raster plot. The voltage distribution is assumed to be Gaussian. The figure is adapted from 44 .

Although the Gauss-Rice approach provides an alternative to derive linear response functions analytically, it should be noted that the formula is a good approximation to the exact linear response function only when the voltage and voltage derivative distribution have close to Gaussian statistics. If the background noise has a large mean and a small variance, the real voltage distribution will be highly skewed, which is not well approximated by Gaussian distributions. Furthermore, in [54], it was shown that the Gauss-Rice approach is a good approximation when the correlation time of the input is close to the membrane time constant of the model. Intuitively speaking, if the correlation time is too small, then with the same stimulus, there might be more spikes counted in the case of no-reset condition. This is because the voltage will fluctuate around the threshold. While having a reset will make the voltage have less likely to fluctuate around the threshold. Gauss-Rice approach will provide additional spikes which undermine the encoding ability at high frequency. On the other hand, when the correlation 
time of the input is much larger than the membrane time constant, GaussRice assumption make the neuron model have a better encoding ability, and overestimates the high bandwidth encoding.

\subsection{Three Hypotheses on the Impact of Neuron Mor- phology and Biophysics on Population Encoding}

Up to now, I have shown that animals can do fast information processing on the behavioral level. Based on the observation that cortical neurons are driven by highly fluctuating inputs, and that cortical neurons are weakly correlated, population firing rate encoding was introduced. Several experimental studies have shown that cortical neurons can realize high bandwidth encoding with the population firing rate. The encoding ability is enhanced when the correlation time of the input is increased. Theoretical studies have found that neuron models with rapid AP onset can realize ultrafast population encoding, although it is not clear whether these two features are equivalent with each other.

In cortical neurons, what are the underlying mechanisms that make the neurons realize fast population encoding? Several hypotheses have been proposed. In this part, I will give an introduction to these hypotheses. Following the progress of previous work, I will raise my scientific questions.

\subsubsection{Axonal Hypothesis}

Action potentials are generated in the axon initial segment (AIS) separated from the soma 5560 . As shown in Fig 13 , synaptic inputs from the dendrites are integrated in the soma and transmitted down to the AIS. The AIS is 15 to $40 \mu \mathrm{m}$ from the soma depending on the neuron type 6165 . The two major ion channels associated with the AP generation at the AIS are sodium and potassium channels. The sodium channels shape the spike initiation. For pyramidal neurons, the sodium channels distributed in the distal AIS are found to have a lower threshold which makes the AIS the location for AP initiation [66. The potassium channels that regulate spike duration are distributed in the more distal part of the AIS 67]. Some studies propose that the AIS has a much higher ion channel densities compared to the soma so that the action potential generation is in the AIS 66 68. The sodium channel density is proposed to be 50 times larger than that in the soma 68. Similarly, the potassium channel density is proposed to be 10 times larger 67]. Some other studies draw different conclusions. With high-speed fluorescence sodium imaging, it was shown that the action potential-associated sodium influx in the AIS is only three times larger than that in the soma 69. Another recent study showed that without high ion channels densities in the AIS, the AP is still initiated in the AIS 70. The AP timing however was impaired in this condition. So still a higher ion channel density is considered beneficial for precise AP timing rather than determining the AP initiation site.

With the separation of AP initiation site from the soma, the synaptic inputs integrated in the soma decay along the transportation down the axon. The high frequency components of the synaptic current are filtered more than the low frequency components. Action potentials are generated with the filtered current entering the AIS. Experimental recordings of the action potentials usually are obtained in the soma. The somatic spike shape and timing can deviate from that in the AIS. So it is important to understand the 
impact of separation of AP initiation site on spike generation dynamics and spike timing detection, which further determine the population encoding of cortical neurons. Several modeling works simplify the neuron as a single compartment soma omitting the detailed structure that might affect AP initiation and population encoding.

Previous studies 48,51] proposed that the separation of the AP initiation site has a big impact on shaping sharp spikes in the soma and directly leads to fast population encoding. Brette implemented a simple multicompartment model composed of a soma and an axon, with a standard sodium activation function at the AP initiation site. When moving the AP initiation site away from the soma, the lateral current entering the soma rises more rapidly as a function of somatic voltage. A larger separation implies a larger resistance between the soma and the AP initiation site. Increasing the clamped somatic voltage will trigger a sodium current entering the AIS. When the sodium current is large enough, the lateral current will change the direction entering the soma. So for the same amount of sodium current entering the AIS, a larger resistance can make the axonal voltage reach a higher value for the same somatic voltage, which leads to more sodium current entering the AIS. The positive feedback results in a new equilibrium point of axonal voltage which triggers a large amount of lateral current. For the spikes seen at the soma, the somatic voltage derivative is influenced by this lateral current. A sharper spike is observed at the soma when the lateral current rises rapidly. Furthermore, representing the sodium activation function as the function of somatic voltage, this function becomes steeper when the AP initiation is farther away from the soma. The sodium activation function seen at the soma can be similar to that of the cooperative gating sodium activation function [46]. Based on this observation, it was proposed that fast population encoding can be realized. The linear response function of related neuron models however have not been calculated directly.

\subsubsection{Cooperative Gating of Sodium Channels Hypothesis}

Several types of ion channels are found to operate in a cooperative gating manner, which means ion channels are not opening or closing independent of the state of other ion channels $72 \sqrt{75}$. For example, the sodium channels in rat and rabbit cardiac myocytes can open and close in double and triple units simultaneously 72. Depending on the channel open probability, the potassium channels from the soil bacteria streptomyces lividans operate in different gating modes. In the low open probability condition, two potassium channels have been observed to activate together. In the high open probability condition, up to five channels have been observed to be coupled for activation 73]. Similar cooperative gating phenomena have also been found for calcium channels in skeletal muscle 74 and nicotinic acetylcholine receptors 75 . Fig 14 shows an example of cooperative gating from 73

The cooperative gating hypothesis focuses on the AP activation dynamics. Cortical neurons have rapid AP onset dynamics [7. Traditional conductance-based models however often fail to reproduce this property $[5,6]$. In the traditional modeling assumption, the sodium channel activation dynamics are independent of each other, which means the rising slope of the total sodium current is the same as that of the standard activation slope of individual sodium channel current. To explain the rapid AP onset, one 


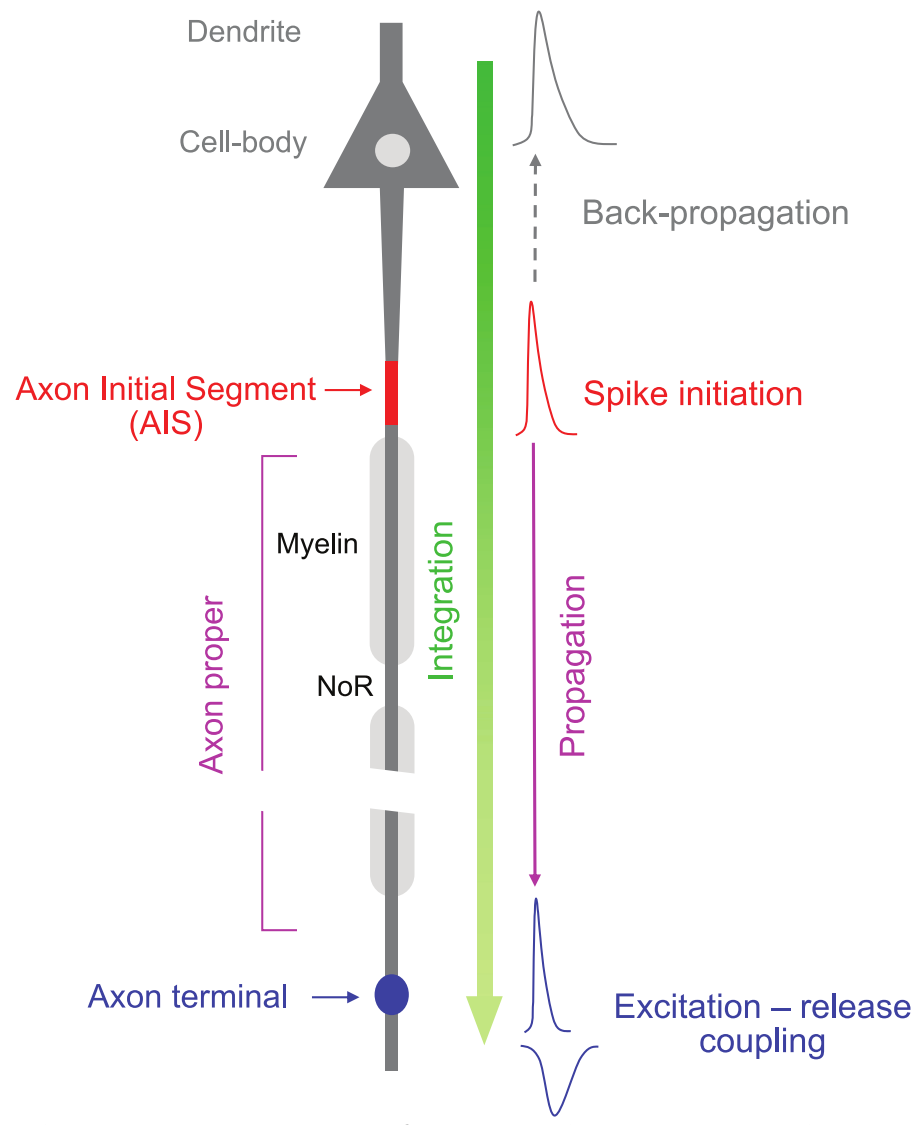

Fig 13. Morphology of a pyramidal neuron with a myelinated axon The synaptic inputs are integrated in the soma and transmitted to the axon, indicated by the green arrow. Action potentials are generated in the AIS separated from the soma and conducted by the myelinated axon. The action potentials seen at the soma are from the AIS. The figure is from 71 .

hypothesis proposed that a group of highly clustered sodium channels may operate in a cooperative way 46]. The activation of one sodium channel may increase the activation probability of its neighboring sodium channels. In this sense, the collective sodium current rises more rapidly than independent sodium channel current. In a theoretical study [45, by tuning the activation slope of the piece-wise linear I-V curve, the model mimicked the rapid collective sodium current, and the influence on the linear response function was obtained analytically. In a subsequent theoretical study 9, the impact of rapid collective sodium current was compared across different neuron models.

A cooperative gating model was built based on this hypothesis in [46] Fig 15 shows the sodium activation function of the cooperative gating sodium channels. The parameter $\lambda$ describes how strong the neighboring channels are affected by the activated channel. Based on the assumption of cooperative gating, when the voltage is low, few sodium channels are activated, the collective sodium current was well approximated by sodium current of independent channels. The sodium activation functions in the figure have a similar rising speed when $V$ is small. For larger $V$ values, a 

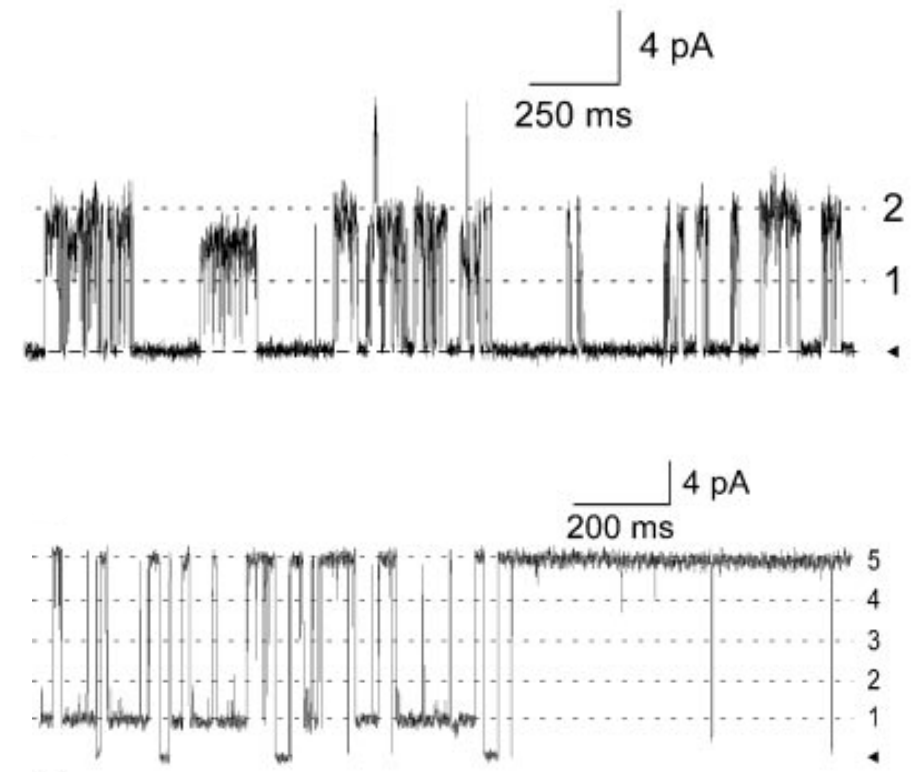

Fig 14. Cooperative gating of potassium channels. The upper figure is the low open probability condition. The lower figure is the high open probability condition. The current axis is divided by the dash lines. Each piece is $4 \mathrm{pA}$, which is the current of one potassium channel. In the low open probability condition, potassium current are dominated by $8 \mathrm{pA}$, which indicates two potassium channels are cooperatively activated. In the high open probability condition, potassium current are dominated by $20 \mathrm{pA}$, which indicates five potassium channels are cooperatively activated. The figure is adapted from 73 .

strong cooperative coupling will trigger more neighboring sodium current, which makes the sodium activation function rises more rapidly.

In 46], adding cooperative gating of the sodium channels enhanced the encoding ability of the conductance-based model in high frequency regime. The enhancement was limited when the percentage of cooperative gating channels is small. The sensitivity of the dynamic gain to the correlation time of the input remains to be examined. The results above indicate that to realize high bandwidth encoding, a strong enough cooperative gating of the sodium channels is required. The ratio of the cooperative gating channels should also be high enough. In a later work, setting cooperative gating model into a multi-compartment framework, sharp spikes at the soma could be reproduced [53]. The impact of cooperative gating on the linear response function was not checked in this multi-compartment model.

\subsubsection{Dendritic Load Hypothesis}

Dendrites integrate synaptic inputs from other neurons 76,77$]$. The integrated inputs are transmitted to the soma and further determine the spiking behaviors at the AIS. The role of the dendrites in encoding is not merely a linear summation of different synaptic inputs 78,79 . Depending on the biophysical properties, the impact of dendrites can be categorized in following a few aspects. First is the stimulus transmission in the passive 


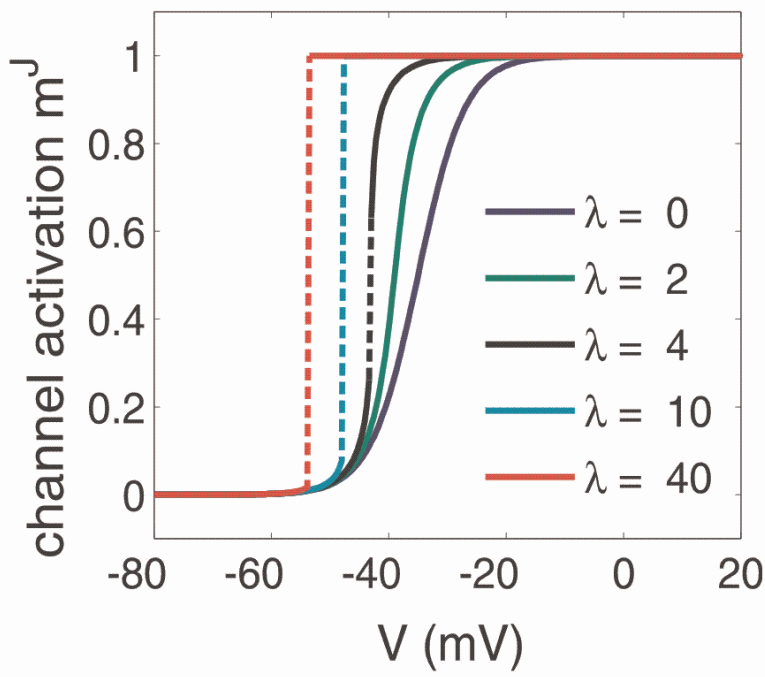

Fig 15. Sodium activation functions with cooperative gating. $V$ is the membrane voltage. $m^{J}$ is the activation probability of the cooperative gating channels. $\lambda$ describes the coupling strength with the neighboring sodium channel. There is no cooperative gating when $\lambda$ is equal to 0 . When $\lambda$ is large, the sodium activation function rises more rapidly. The figure is from 46 .

dendrites [80]. High frequency fluctuations in the synaptic inputs are more attenuated when transmitted down to the soma. Also, the synaptic input from the more distal end of the dendrites has a longer time delay to reach the soma. Second is the summation of the excitatory and inhibitory inputs under different input locations 81]. Two excitatory inputs close to each other reduce the effect of each other. The total impact of the two is a sub-linear summation. In contrast, two excitatory inputs separated from each other have a linear summation. The existence of shunting inhibition changes the membrane conductance without changing the voltage, which reduce the impact of excitatory inputs 80,82 . Third is the impact of active ion channels in the dendrite on the dendrite computation. Synaptic inputs can trigger more ion currents entering the soma, which may amplify the synaptic inputs and affect the spike timing 83 85. For some neuron types, it is even possible to have dendritic action potential transmitted to the soma. 86 88 This type of behavior is classified as supra-linear. Reversely, the action potentials generated at the AIS can also interact with ion channels in the dendrites.

Recently, it has been proposed that the computation ability in the dendrites may impact the population encoding ability of cortical neurons 49. Without any explicit requirements on the AP generation dynamics, implementing a multi-compartment model composed of a dendrite, a soma and an axon, high bandwidth encoding was observed. The cutoff frequency of the linear response function was above $100 \mathrm{~Hz}$, which was far larger than the firing rate of the neuron model. Increasing the dendrite size enhanced 
the encoding ability of the neuron model. One interesting observation of the linear response properties was that even without the dendrite, the multicompartment model was also realizing a high cutoff frequency of the linear response function. The linear response function had a resonance at around $50 \mathrm{~Hz}$. The resonance is smaller but maintained when adding a dendrite to the neuron model. Compared with the simpler multi-compartment model proposed in the axonal hypothesis 48 , this model has a complete AP generation mechanism which includes sodium and potassium channels. The inactivation of sodium channels was also considered. The soma was also equipped with ion channels. The results above indicate that high bandwidth encoding can be realized without cooperative gating of sodium channels. However, this work does not examine the sensitivity of the linear response function to the correlation time of stochastic input, so it remains unclear whether the neuron model can reproduce fast population encoding in cortical neurons.

In a subsequent work, the impact of dendrite morphology on population encoding was studied in Purkinje cells. The Purkinje cells are inhibitory neurons of the cerebellum, which have very large dendrites. Experiments showed that the linear response function of Purkinje cell displayed a strong resonance around $200 \mathrm{~Hz}$ under white noise stochastic stimulation combined with a sinusoidal signal. The cutoff frequency of the linear response function even approached $1000 \mathrm{~Hz}$. The resonance in the linear response function was proposed to be introduced by the large dendrite. In the theoretical modeling part, a two-compartment model composed of a big dendrite and a soma was shown that increasing the dendrite size or reducing the soma size can further enhance the resonance in high frequency regime as we can see in Fig 17. But this effect was restricted to the condition that the sinusoidal signal is injected at the soma and the background noise injected in the dendrite. Injecting the sinusoidal signal in the dendrite or injecting both the signal and the background noise to the soma failed to reproduce high frequency resonance as shown in Fig 18 .

Two aspects of this work require further exploration. One is the examination of the sensitivity of the dynamic gain to the correlation time of the input. It remains to check whether the high bandwidth encoding here is fast population encoding. Second there is a seeming contradiction on the impact of soma size between the axonal hypothesis model and the model for the Purkinje cell. In the axonal hypothesis, we have a big soma and a thin axon. The AP is initiated in the AIS. As we will show latter, with a step-like sodium activation function, reducing the soma size can enhance the encoding ability. The two-compartment model has a big dendrite and a soma. The AP is initiated in the soma. We can see a mapping between the morphologies of these two models. However, in the second case, increasing the dendrite can enhance the encoding ability, which seems to be in contradiction with the first result. Further explorations on the underlying mechanisms of two separate observations are thus required.

\subsection{A Brief Summary of My Work}

In chapter two, I will first introduce the numerical methods for calculating the linear response functions, which include the Fourier transform method and the vector strength method. I will show that the linear response functions obtained with these two methods fit with each other. Then I will introduce the bootstrapping and null hypothesis test which tell us the 

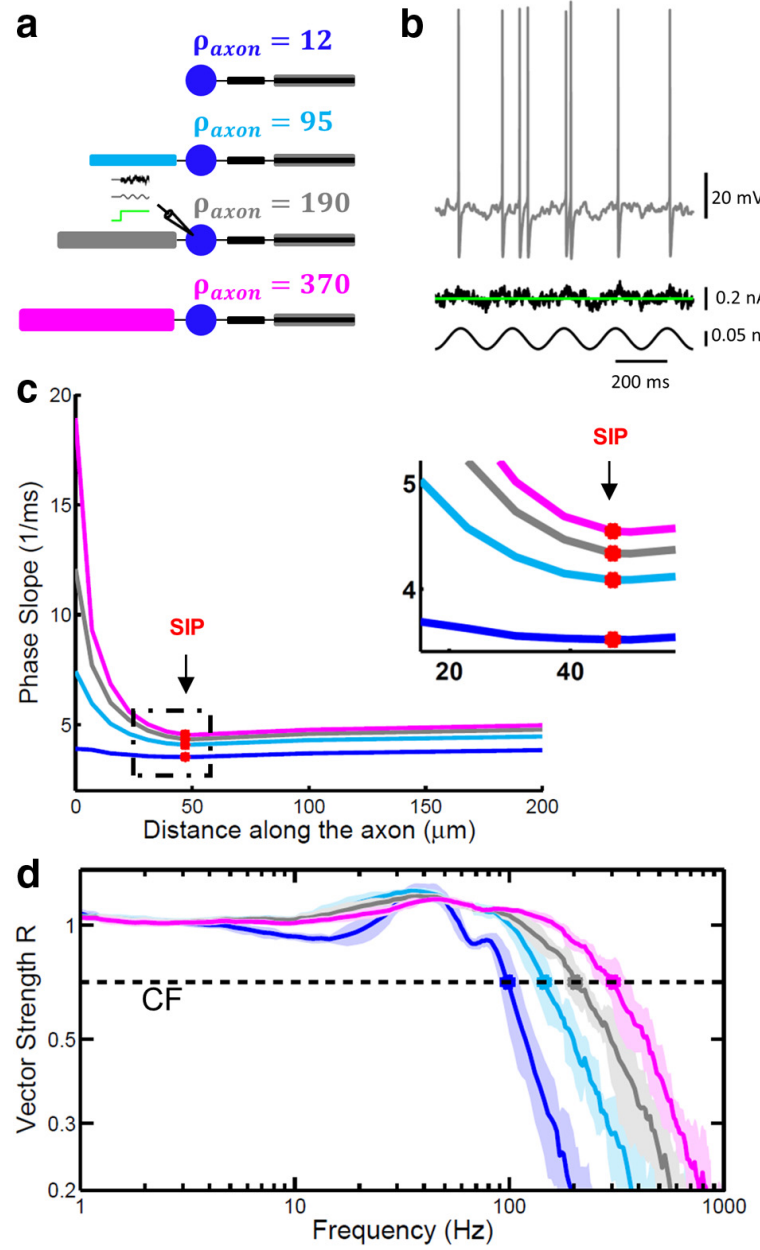

Fig 16. The impact of dendrite size on AP onset and population encoding. a) shows the neuron models with different dendrite sizes. $\rho_{\text {axon }}$ describes the conductance ratio between the dendrite plus the soma and the axon. b) shows the stimulus setup for calculating linear response function. Injecting the soma with a small sinusoidal signal merged in stochastic stimulus, the tuning ratio between the firing rate fluctuation amplitude and the sinusoidal amplitude is the dynamic gain at related frequency. c) shows the AP onset rapidness of different models. d) shows the linear response functions of different models. Based on c) and d), a larger dendrite enhances the onset rapidness and the encoding ability. The figure is adapted from 49 .

confidence interval and frequency credibility region of the dynamic gain. In the last part of the chapter, I will show that previous work of evaluating the AP onset rapidness as the slope at some specific point on the phase plot or the I-V curve is inaccurate to indicate the encoding ability of the neuron model. The AP generation dynamics and the stimulus together determine the time it takes from spike initiation to spike detection. The time delay in between acts as a low pass filter of the linear response function. Without the spike detection delay, the encoding ability of the neuron model is similar to the LIF model. One simple parameter to quantify the random variable 

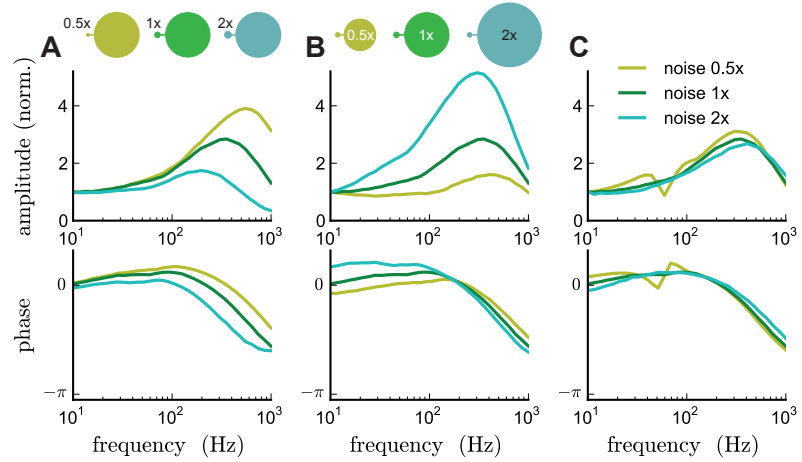

Fig 17. The impact of soma and dendrite size on population encoding in Purkinje cells. A. Increasing the soma size reduces the dynamic gain in high frequency regime. The resonance frequency is decreased with the soma size. B. Increasing the dendrite size enhances the dynamic gain in high frequency regime. The resonance frequency is slightly reduced with dendrite size. C. Increasing the strength of stochastic fluctuations has week effects on linear response functions. The figure is from 52 .
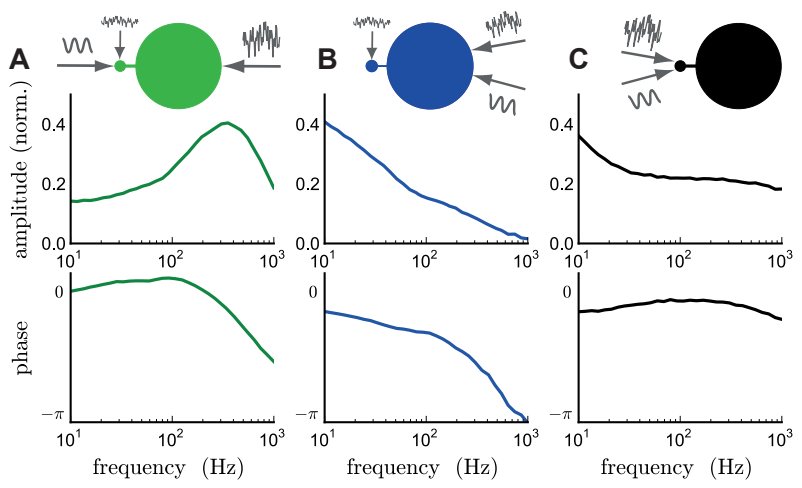

Fig 18. The linear response functions under different locations of signal and noise injection. The high bandwidth and high frequency resonance are realized only when the signal is injected in the soma and the stochastic fluctuations is injected in the dendrite. As it is shown in A. Injecting the signal in the dendrite or injecting the fluctuations only in the soma fail to reproduce high bandwidth encoding. The figure is from 52.

of the spike detection delay is its std.

In chapter three, I will examine the axonal hypothesis which proposes that rapid AP onset and fast population encoding can be realized by separating the AP initiation site from the soma [48]. I will calculate the linear response function of the neuron model and show that with a standard sodium activation function, the linear response function has a low cutoff frequency at the range of the firing rate. The dynamic gain of the linear response functions in the high frequency regime is not sensitive to the correlation time of the input. Replacing the sodium activation function with a step-like function can fix these two problems. In both conditions, no clear impact of the AP initiation site is observed. By comparing the relations between the somatic and axonal voltage during AP generation, 
I propose that suppression of the sodium current before AP initiation is crucial for realizing fast population encoding. In this sense, the sodium activation dynamics have high voltage sensitivity during AP generation. In the last part of this section, I simplified the multi-compartment model to a single compartment model. With the Gauss-Rice approach, I obtain the linear response function analytically when the sodium activation function is a step-like function. Based on the formula, it can be seen how the dynamic gain is sensitive to the soma size.

In chapter four, I will examine the cooperative gating model which claims to realize fast population encoding without requiring suppression of the sodium current explicitly before AP initiation [46]. I will first show that with the parameters given in the paper, it is not able to reproduce the linear response functions. The linear response function has a low cutoff frequency. The dynamic gain is not sensitive to the correlation time of inputs. Increasing the ratio and cooperativity of the sodium channels will make the neuron model not able to generate spikes properly. Adding cooperative gating to the sodium inactivation can fix the problem of spike generation, but the neuron model still can't realize fast population encoding. In comparison, if we design a piece-wise linear sodium activation function which suppresses the sodium current before AP initiation, without too high activation slope, we can reproduce the fast population encoding in the multi-compartment model investigated in [48. We can also observe the impact of AP initiation site on the encoding ability. The AP initiation site determines the lateral current entering the soma during AP initiation, which changes the AP onset rapidness when the sodium activation dynamics are not too voltage sensitive. As the last part of this section, I will also examine the impact of soma size on the encoding ability numerically. With a smaller soma, the encoding ability of the neuron model is enhanced.

In chapter five, I will examine the encoding ability of the neuron model proposed in 49. This work seems to indicate that fast population encoding can be realized without requiring rapid AP onset or cooperative gating of the sodium channels. I will first reproduce the high bandwidth encoding. Then I will show that the dynamic gain in the high frequency regime isn't sensitive to the correlation time of the input, which implies that the neuron model can't reproduce fast population encoding as found in the experiments [36]. By calculating the F-I curve, I find that the neuron model is of type II excitability capable of high frequency repetitive firing. When responding to the stochastic stimulus, the neuron model is more likely to generate bursts of spikes. The dynamic gain values near the burst firing frequency are selectively enhanced, which results in the high bandwidth encoding. In the last part of this chapter, I will show that an active soma with ion channels in the multi-compartment model, and the relative position of the sodium and potassium channels along the voltage axis determine the ability of high frequency repetitive firing of this neuron model. 


\section{Methods}

In this chapter, I will introduce the numerical methods for calculating linear response functions. The introduction is based on the multi-compartment model in 48, which will be extensively studied in following chapters. In the first section of this chapter, I will give an overview of the multi-compartment model, which includes the model morphology, biophysical parameters, and ion channel dynamics. In the second section, I will talk about tuning the parameters of stochastic inputs to obtain the firing rates and firing patterns we need. During this process, the rule of defining spike time and reset voltage to generate a spike will be given. In the third part, I will introduce two numerical methods to calculate the linear response functions. For the first method, the linear response function is obtained with the Fourier transform. For the second method, the linear response function is obtained with vector strength which captures the tuning ratio between the firing rate fluctuation and sinusoidal signal. The comparison between these two numerical methods will be provided. In the fourth part, I will introduce the bootstrapping confidence interval and null hypothesis test, which tell us the reliability of the linear response functions we have calculated. In the last section of this chapter, I will provide a view to evaluate the impact of AP generation dynamics on population encoding. I will show that the traditional way of evaluating AP onset rapidness as the slope of one point in the phase plot might be misleading to indicate the encoding ability. For type I neuron model, the AP generation rapidness corresponds to the dynamics after the local minimum in the phase plot. This part of dynamics provides an uncertainty on spike time detection. Rapid AP onset can reduce the uncertainty and improve the encoding ability. The linear response function can be decomposed into a LIF like part related to the subthreshold dynamics and a low-pass filter related to the AP onset dynamics.

\subsection{Introduction to Brette's Model}

The multi-compartment model is composed of a soma and an axon. The soma is a cylinder of which the diameter and length are both $50 \mu m$ denoted as $d$. The axon is a cylinder, with a diameter of $1 \mu m$ denoted $a$, and a length of $600 \mu \mathrm{m}$ denoted $L$. Fig 19 provides the morphology of the multi-compartment model.

In the aspect of neuron biophysical parameters, following previous work [48], the soma is passive. The specific membrane resistance $R_{m}$ is $30000 \Omega \cdot \mathrm{cm}^{2}$. The specific membrane capacitance $c_{m}$ is $0.75 \mu \mathrm{F} / \mathrm{cm}^{2}$. The longitude resistance $R_{a}$ is $150 \Omega \cdot \mathrm{cm}$. The resting potential is $-75 \mathrm{mV}$. For the axon, the passive biophysical parameters are the same as those of the soma. Sodium channels are inserted at one point in the axon initial segment. The position of the sodium channels is also the position of AP initiation site. The distance between the soma and the AP initiation site is denoted $P_{N a}$. In this work, we set $P_{N a}$ as $20 \mu m, 40 \mu m$, and $80 \mu m$ separately to study its impact on population encoding. Potassium channels and other ion channels are not included. Only the sodium activation dynamics is considered. Inactivation of the sodium channels is not included. To generate a spike, the voltage values across the neuron model are reset to resting potential once a threshold is reached by the voltage at the AP initiation site. The sodium current is represented as $I_{N a}=\bar{g}_{N a} \cdot m\left(V_{a}\right) \cdot\left(E_{N a}-V_{a}\right)$. Here $\bar{g}_{N a}$ is the sodium peak conductance. We set it as $5.23 * 10^{-3} S . m$ is the 


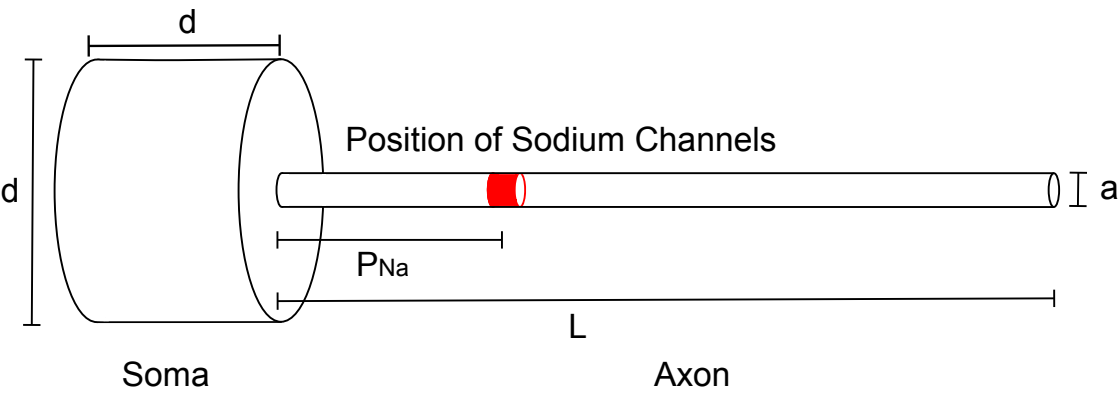

Fig 19. Morphology of the multi-compartment model. The soma is of a cylindrical shape with equal length and diameter denoted $d$. The axon is represented by a tube with a length $L$ and constant diameter $a$. Sodium channels are located at one point in the axon which is marked in red. This is also the AP initiation site. The distance between the soma and the initiation site is denoted $P_{N a}$. The stimulus is injected in the middle of the soma, where also the somatic voltage $V_{s}$ is recorded. The axonal voltage $V_{a}$ is recorded at the AP initiation site.

sodium activation function describing the ratio of sodium channels being activated at the given voltage $V_{a} . V_{a}$ is the voltage at the AP initiation site. $E_{N a}$ is the balanced voltage of the sodium channels set as $60 \mathrm{mV}$. The activation function of sodium channels is represented as:

$$
m\left(V_{a}\right)=1 /\left(1+\exp \left(\left(V_{m}-V_{a}\right) / k_{a}\right)\right) .
$$

The midpoint voltage $V_{m}$ is set to $-40 \mathrm{mV} . k_{a}$ is proportional to the inverse of activation slope at $V_{m}$. A larger $k_{a}$ implies slower sodium activation. When $k_{a}$ is close to zero, the sodium activation function is close to a step function. When examining the axonal hypothesis, we used the standard sodium activation function, where $k_{a}$ is set to $6 \mathrm{mV}$. The activation variable of the sodium channels $m$ will be reset together with the voltage values when a spike is generated. All the parameters above are the default values. New parameter values will be stated when it is changed.

The neuron model equations are as below. Here we use $V_{s}(x, t)$ and $V_{a}(y, t)$ to denote the voltage value somewhere on the soma and on the axon at some time point. The denotations $V_{s}$ and $V_{a}$ refer to the voltage in the middle of the soma and the voltage at the AP initiation site.

For the voltage of soma:

$$
c_{m} \frac{\partial V_{s}(x, t)}{\partial t}=\frac{d}{4 R_{a}} \frac{\partial^{2} V_{s}(x, t)}{\partial x^{2}}-\frac{V_{s}(x, t)-V_{\text {rest }}}{R_{m}}+\frac{I_{e}}{\pi d} \delta\left(x-\frac{d}{2}\right)
$$

$I_{e}$ is the somatic input injected in the middle of the soma.

For the voltage of axon:

$$
c_{m} \frac{\partial V_{a}(y, t)}{\partial t}=\frac{a}{4 R_{a}} \frac{\partial^{2} V_{a}(y, t)}{\partial y^{2}}-\frac{V_{a}(y, t)-V_{\text {rest }}}{R_{m}}+\frac{I_{N a}(y, t)}{\pi a} \delta\left(y-P_{N a}\right)
$$

Where:

$$
\begin{gathered}
I_{N a}(y, t)=\bar{g}_{N a} \cdot m\left(V_{a}(y, t)\right) \cdot\left(E_{N a}-V_{a}(y, t)\right) ; \\
\tau_{m} \frac{\mathrm{d} m}{\mathrm{~d} t}=m_{\infty}-m .
\end{gathered}
$$

The boundary conditions are:

$$
V_{s}(d, t)=V_{a}(0, t)
$$




$$
\begin{gathered}
\left.d^{2} \frac{\partial V_{s}(x, t)}{\partial x}\right|_{x=d}=\left.a^{2} \frac{\partial V_{a}(y, t)}{\partial y}\right|_{y=0} \\
\left.\frac{\partial V_{s}(x, t)}{\partial x}(x, t)\right|_{x=0}=0
\end{gathered}
$$

and

$$
\left.\frac{\partial V_{a}(y, t)}{\partial y}(y, t)\right|_{y=L}=0 .
$$

\subsection{Simulation Setup and Parameter Searching}

In the aspect of simulations, I used NEURON 7.3 and Python 2.6 for all the simulations on multi-compartment models 89 . For single compartment models, Python 2.6 are used. The simulation time step is $0.025 \mathrm{~ms}$, which is the default time step in NEURON. For the multi-compartment introduced above, reducing the time step to $0.005 \mathrm{~ms}$ doesn't make a big difference on the linear response functions. So $0.025 \mathrm{~ms}$ can be regarded as a small enough time step for precise calculation of the linear response functions. For all the other neuron models simulated below, the time step is $0.025 \mathrm{~ms}$ if not stated in specific.

To generate spikes in Brette's model, stochastic stimuli are injected in the middle of the soma. The stochastic stimuli are generated with an Ornstein-Uhlenbeck (OU) process represented by the following equation:

$$
\tau d I_{e}=-\left(I_{e}-I_{\text {mean }}\right) d t+\sqrt{2 \tau} \sigma d W(t)
$$

$\tau$ is the correlation time of the stimulus $I_{e}$. The mean and the std of $I_{e}$ are denoted $I_{\text {mean }}$ and $\sigma$ respectively. $W(t)$ is the Wiener process satisfying $W_{t+d t}-W_{t} \sim N(0, d t)$.

In order to study the impact of different neuron and stimulus parameters on the linear response function, it is expected that the neuron models in different parameter settings are firing at similar working point so that simulation results are comparable. So here we use the firing rate and the firing irregularity, which is the coefficient of variation (CV) of the inter spike intervals (ISI), to quantify the working point of the neuron model. In this way, there exists a unique pair of mean and std of the stimuli to reproduce the required firing rate and CV (of ISI). For Brette's model, the mean and the std of stimuli are tuned to reproduce a mean firing rate of about $5 \mathrm{~Hz}$. CV is controlled to be around 0.85 .

To find the mean and the std, it is necessary to determine the voltage that indicates the spike time and the voltage for reset to finish a spike. We call these two voltages as spike time threshold and reset threshold. Since the spikes are generated at the AP initiation site, these two voltage values are defined based on $V_{a}$. To define the spike time threshold, we first set reset voltage to $60 \mathrm{mV}$, so that there is no spikes if the injected stimulus is not too big. Injecting the soma with a constant input, we choose the voltage at which the voltage derivative reaches its maximum as the spike time threshold. The spike time threshold is insensitive to the stimulus amplitude if it is not too high. Temporarily choosing spike time voltage as the reset threshold, we can find a constant input to generate $5 \mathrm{~Hz}$ firing rate. In the simulation, setting the neuron firing time to be 20 s, we manually choose a upper bound and a lower bound for the constant input. Injecting the neuron with the average of the upper bound and the lower bound, if the firing rate is larger than $5 \mathrm{~Hz}$, the average will be set as the new upper 
bound. Otherwise the average will be set as the new lower bound. The iteration above will end if the difference between the upper bound and the lower bound is smaller than $0.1 \%$ of the maximum of absolute values of the upper bound and the lower bound. Similarly, we can set the target firing rate to $0 \mathrm{~Hz}$ to find the constant input that is about to trigger spikes. To mimic the time it takes to finish a spike and the following refractory period, we choose the voltage $2 \mathrm{~ms}$ after the spike time voltage under the constant input that generate $5 \mathrm{~Hz}$ firing rate as the new reset threshold.
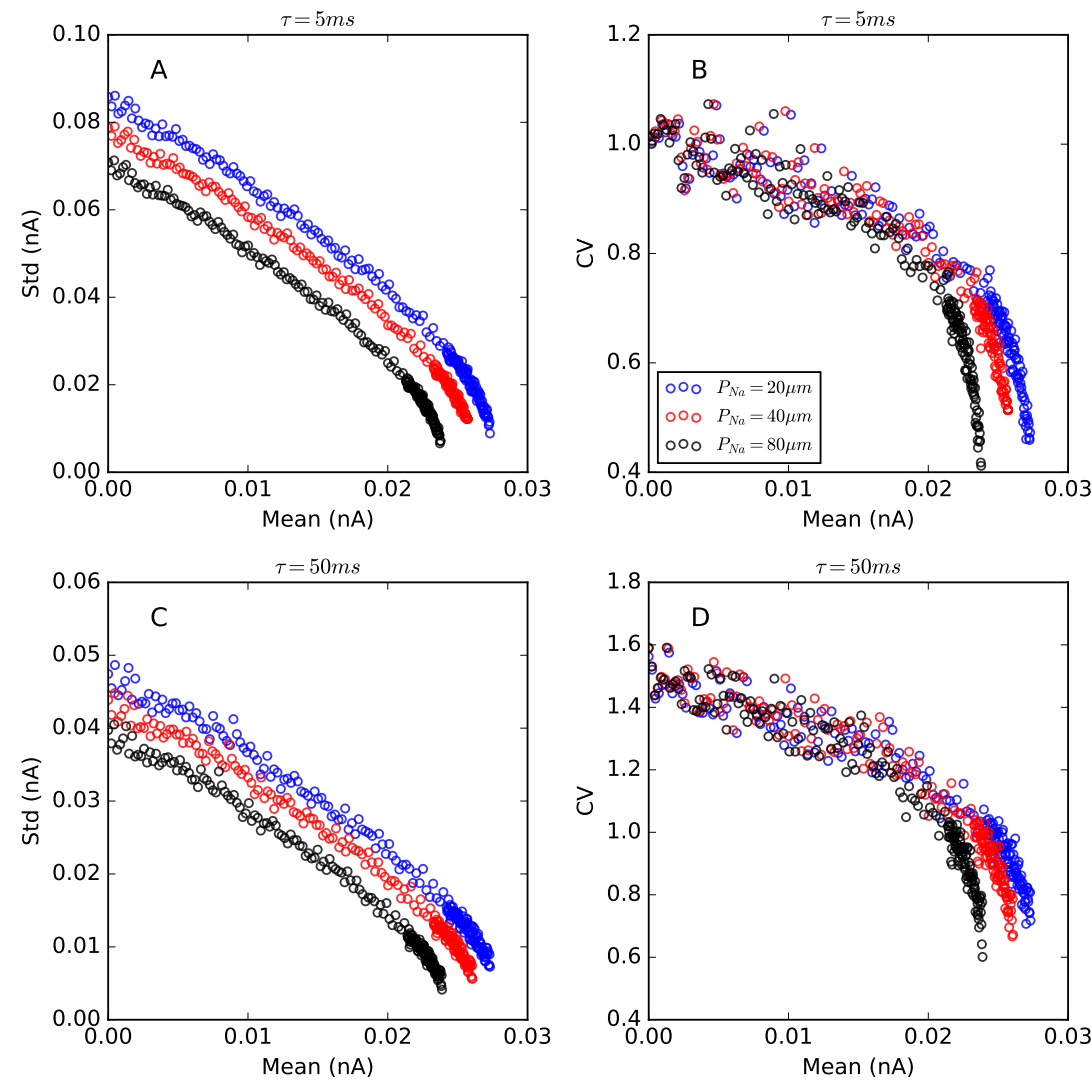

Fig 20. Mean-std, and mean-CV relation for Brette's model. In the first row and the second row, the correlation times of the input are set to $5 \mathrm{~ms}$ and $50 \mathrm{~ms}$ separately. The color codes for all figures are given in B. $k_{a}=6 \mathrm{mV}$. When the sodium activation is slow, it requires less current to generate the same firing rate when AP initiation site is further away from soma. The mean-CV decays faster as well. The mean-CV relation is more noisy than mean-std relation.

Up till now, we have determined the spike time threshold and reset threshold of the neuron model. Now we will search for the mean and std of the stimulus to reproduce the expected firing rate and CV. In this step, for a give mean, we first search for the std such that the firing rate is about $5 \mathrm{~Hz}$. The searching method is the same as above. The simulation time is increased to 200s. The precision is changed from $0.1 \%$ to $1 \%$. Then for different values of the mean, we can get the related std which reproduce the firing rate. For each pair of mean and std, we can estimate the CV 
with 200s simulation, so that we obtain the plot of mean and CV. Fig 20 is an example of the mean-std and mean-CV relation for Brette's model. For each color code, we choose 200 scatter points. In the interval from the constant to trigger spikes to the constant to generate $5 \mathrm{~Hz}$ firing rate, the $\mathrm{CV}$ is changing faster with the mean, so we have 100 points in this region. When the mean is small, the mean-std relation is close to linear. When the mean is close to the constant input that is about to trigger spikes, the mean-std relation is becoming nonlinear. As for the mean-CV relation, it is more noisy than the mean-std curve. It is hard to fit a clear curve for the mean-CV relation. When the mean is close to the constant to trigger $5 \mathrm{~Hz}$ firing rate, $\mathrm{CV}$ decays quickly to zero. This indicates that the firing pattern is converge to regular firing. When the correlation time of the input is increased to $50 \mathrm{~ms}$, the mean-std relation and mean-CV relation are more noisy than the case of $5 \mathrm{~ms}$. But still we can observe similar relations. Based on the mean-CV plot, we can pick the mean value associated with the required $\mathrm{CV}$. Then in the mean-std plot, we can find corresponding std.

\subsection{Two Methods for Calculating Linear Response Func- tions}

In this section, I will introduce two numerical methods for calculating the linear response functions. I will also compare the performance of these two methods.

For the first method, the linear response function is calculated with the Fourier transform of the spike triggered average (STA) divided by the power spectral density of the OU process $[9,35]$. The Fourier transform of the STA is the Fourier transform of the cross correlation of the input stimulus and the output spike times. Power spectral density is the Fourier transform of the auto correlation of the input stimulus. The ratio of the two describes the tuning ability across different frequencies, which is exactly the linear response function. Fig 21 is an illustration of the simulation steps based on Brette's model. Here the position of the sodium channels is $20 \mu \mathrm{m}$ from the soma. The correlation time of the input is $5 \mathrm{~ms}$. The first two panels give an example of the stimulus and firing patterns. In this case, the spike time threshold is $-35 \mathrm{mV}$. The reset threshold is $-23 \mathrm{mV}$. The spike time is determined as the time bin that axonal voltage crosses the spike time threshold from below. The STA is obtained by averaging stimulus intervals centered at the spike times. In the simulations of Brette's model, the stochastic stimulus is of 20.5 seconds with the initial value as $I_{\text {mean }}$. The first 0.5 seconds are for randomizing the initial condition. We used the spikes that are emitted in the following 20 seconds and have a complete STA in this period of time to calculate the STA. The duration of the STA is 0.8 seconds. During the simulation, we generated 400 pieces of data. Each piece of data contains 50 realizations of the 20 seconds stimulus and the spike times. From each piece of data, we obtained a STA from 5000 spikes. The final STA for Fourier transform is an average of 400 such STAs. In total, the STA is obtained by averaging 2 million pieces of stimuli centered at the spike times. As for the power spectral density of the OU process, it can be calculated analytically.

The figures in the third panel of Fig 21 show the Fourier transform of the STA and the power spectral density of the stimulus. The Fourier transform components are complex numbers. Here we show the absolute values. We can see that in the high frequency regime, the Fourier components become 

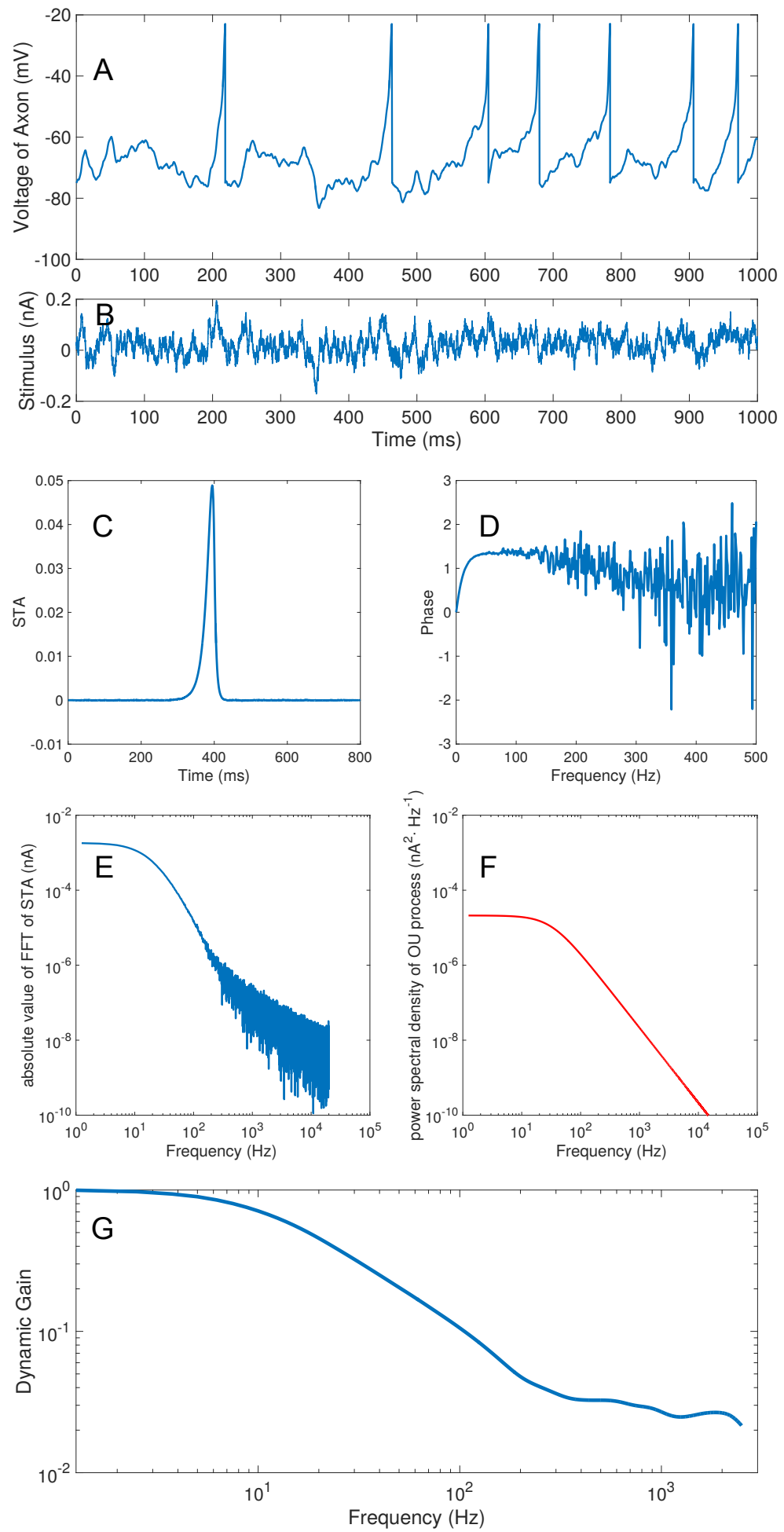

Fig 21. Calculation of the linear response function with Fourier transform. A and B are an illustration of the stimulus and firing patterns of the neuron model. $\mathrm{C}$ is the spike triggered average. $\mathrm{D}$ is the phase shift of the Fourier transform of STA cutting from the middle. E and F are the amplitude of the Fourier components and power spectral density of the stimulus. $G$ is the linear response function after the filtering by Gaussian filters. The noise in high frequency regime is smoothed out. 
very noisy. In fact, the phase shift of related components are also dominated by the noise which are highly fluctuated. To smooth out the noise in high frequencies, each complex Fourier component of the STA is filtered by a Gaussian filter centered at its frequency. The variance of the filter is chosen to be scaled with the frequency. For low frequency components, the noise is very small. The variance of the Gaussian filter is small, so that the filtered components are close to the original components. For higher frequency components, the variance of the Gaussian filters increases with the frequency so that the noise terms can be averaged out. The filtering equation can be expressed as below.

$$
f t S T A_{n e w}[i]=\sum_{j=1}^{n} f t S T A[j] \frac{e^{-2 \pi^{2} \cdot \frac{(f[j]-f[i])^{2}}{f[i]^{2}}}}{\sum_{k=1}^{n} e^{-2 \pi^{2} \cdot \frac{(f[k]-f[i])^{2}}{f[i]^{2}}}}
$$

The ith term of the filtered Fourier transform of STA is denoted as $f t S T A_{\text {new }}[i]$. The $j$ th term of the original Fourier transform of STA is denoted as $f t S T A[j]$. The fractional part multiplied with $f t S T A[j]$ is a discrete representation of a Gaussian distribution. The variance of the distribution is correlated with ith term in frequency components. For small $i$, the variance is small. The $f t S T A_{\text {new }}[i]$ is almost the same as the old one. For large $i$, the $f t S T A_{\text {new }}[i]$ is an average of a large range of old $f t S T A[j]$ values. The last figure of Fig 21 is a representation of the linear response function filtered by the Gaussian filters. The gain curve is normalized by its first component.

Two potential problems should be pointed out for this method. When we apply the Gaussian filtering to the Fourier transform of the STA, it is expected that the phase shifts in the low and intermediate frequency regimes are small such that the filtering doesn't average out the dynamic gain. So before the Fourier transform, it is necessary to consider where to cut the STA and attach them with two ends. Here we cut the STA from the middle. From the phase shift plot we can see that there is no drastic phase change in corresponding frequency regimes. The second problem is the Gaussian filter. For small correlation time of the stimulus, the filtered dynamic gain looks smooth with the frequency. However, for the large correlation time of the stimulus, the dynamic gain is highly fluctuated. The fluctuation is an artifact of the filtering. Also, in the limit of large frequencies, the size of the filter is very large, which may average out the trend of the dynamic gain. So it is required to figure out the confidence interval of the linear response function. In the next section, I will talk about the bootstrapping and null hypothesis test which tells us up to which frequency the linear response function is reliable enough.

The second method is based on the observation that cortical neurons can represent the sinusoidal signal merged in the background noise with population firing rates 9,33 . The population firing rate is also in the form of sinusoidal fluctuations. The tuning ratio between the population fluctuation amplitude and the sinusoidal signal amplitude is the dynamic gain of related frequencies. In the experimental recording or the simulations, it may require large amount of spike times to average our the noise and obtain the sinusoidal population firing rate fluctuations. This problem is more serious for high frequency signals since the population firing rate fluctuations are getting smaller. In [44, it pointed out an alternative way to get around this problem. The fitting of the sinusoidal function can be substituted with averaging over the complex vectors generated with the 
spike times. Below is an illustration of this idea. Assume $t_{1}, t_{2}, \ldots, t_{N}$ are the spike times of the neuron to $\mathrm{K}$ trials of sinusoidal signals merged in background noise. The time interval for each trial is $\mathrm{T}$. The averaged firing rate for one trial is denoted as $\nu(t)$. Based on the linear response property, $\nu(t)$ can be expressed as $\nu(t) \approx \nu_{0}+A \nu_{1}(\omega) \cos (\omega t-\phi) . \nu_{0}$ is the mean firing rate satisfying $N=K T \nu_{0} . \nu_{1}(\omega)$ is the linear term of the sinusoidal fluctuation. $\phi$ is the phase shift between the firing rate and the original signal. Then for $r=\sum_{i=1}^{N} \exp \left(i \omega t_{i}\right) / N$, it is equivalent to:

$$
r=\frac{1}{N} \int_{0}^{T} \exp (i \omega t) \cdot \sum_{i=1}^{N} \delta\left(t-t_{i}\right) d t
$$

$\sum_{i=1}^{N} \delta\left(t-t_{i}\right)$ is the total firing rate of $\mathrm{K}$ trails of stimuli, which can be approximated as $K \cdot\left(\nu_{0}+A \nu_{1}(\omega) \cos (\omega t-\phi)\right)$. Then:

$$
\begin{aligned}
r & =\frac{K \nu_{0}}{N} \int_{0}^{T} \exp (i \omega t)\left(1+\frac{A \nu_{1}}{\nu_{0}} \cdot \cos (\omega t-\phi)\right) d t \\
& =\frac{1}{T} \int_{0}^{T} \exp (i \omega t)\left(1+\frac{A \nu_{1}}{\nu_{0}} \cdot \cos (\omega t-\phi)\right) d t \\
& =\frac{A \nu_{1}(\omega)}{2 \nu_{0}} \exp (i \phi)
\end{aligned}
$$

For different frequencies of the sinusoidal signals, assume that their amplitudes are small such that they don't change the mean firing rate. Then we have $v_{1}(\omega) \sim \frac{1}{A}\left|\frac{1}{N} \sum_{i=1}^{N} \exp \left(i \omega t_{i}\right)\right|$. Note that $\omega$ is $2 \pi f$ in the simulation. $f$ is the frequency of the sinusoidal signal. Fig 22 is an illustration of the vector strength method.

Applying the vector strength method, we calculated the linear response function of Brette's model and compared it with the Fourier transform method shown in Fig 23. We used the previous stochastic stimulus as the background noise. The firing rate is $5 \mathrm{~Hz}$ with the background noise. We picked the sinusoidal frequencies as the following set: 1, 2, 5, 10, 20, 50, 80, $100,200,400,500,800,1000(\mathrm{~Hz})$. For each sinusoidal frequency, we used the following amplitudes: $1,1 / 2,1 / 5,1 / 10,1 / 20$. For each frequency and amplitude, each trial of the simulation is $20 \mathrm{~s}$ with $0.5 \mathrm{~s}$ of randomization. The simulation time is $10 \%$ of the Fourier transform method, which is 40000 seconds. When calculating the dynamic gain, we averaged the complex vectors of the spike times, then divided with the amplitude. Here we did not normalize it with the firing rates. Fig 23 A shows the linear response functions calculated with different amplitudes of the sinusoidal signals. For low frequencies, a large sinusoidal signal introduces large sinusoidal fluctuations in the firing rate. If the sinusoidal signal amplitude is too big, it might not be able to observe complete sinusoidal firing rate since the firing rate may become zero. Also, if we examine the averaged firing rate of the sinusoidal fluctuated firing rate, it may be different from the averaged firing rate generated with the background noise. This is why the dynamic gain values of the low frequencies calculated with the large amplitudes are smaller than those calculated with the small amplitude signals. When the sinusoidal amplitudes are small enough such that they have limited impact on the average firing rate, the dynamic gain values are aligned with each other. However, when the sinusoidal signal is too small, it requires more data to average out the noise. As shown in the figure, the linear response 

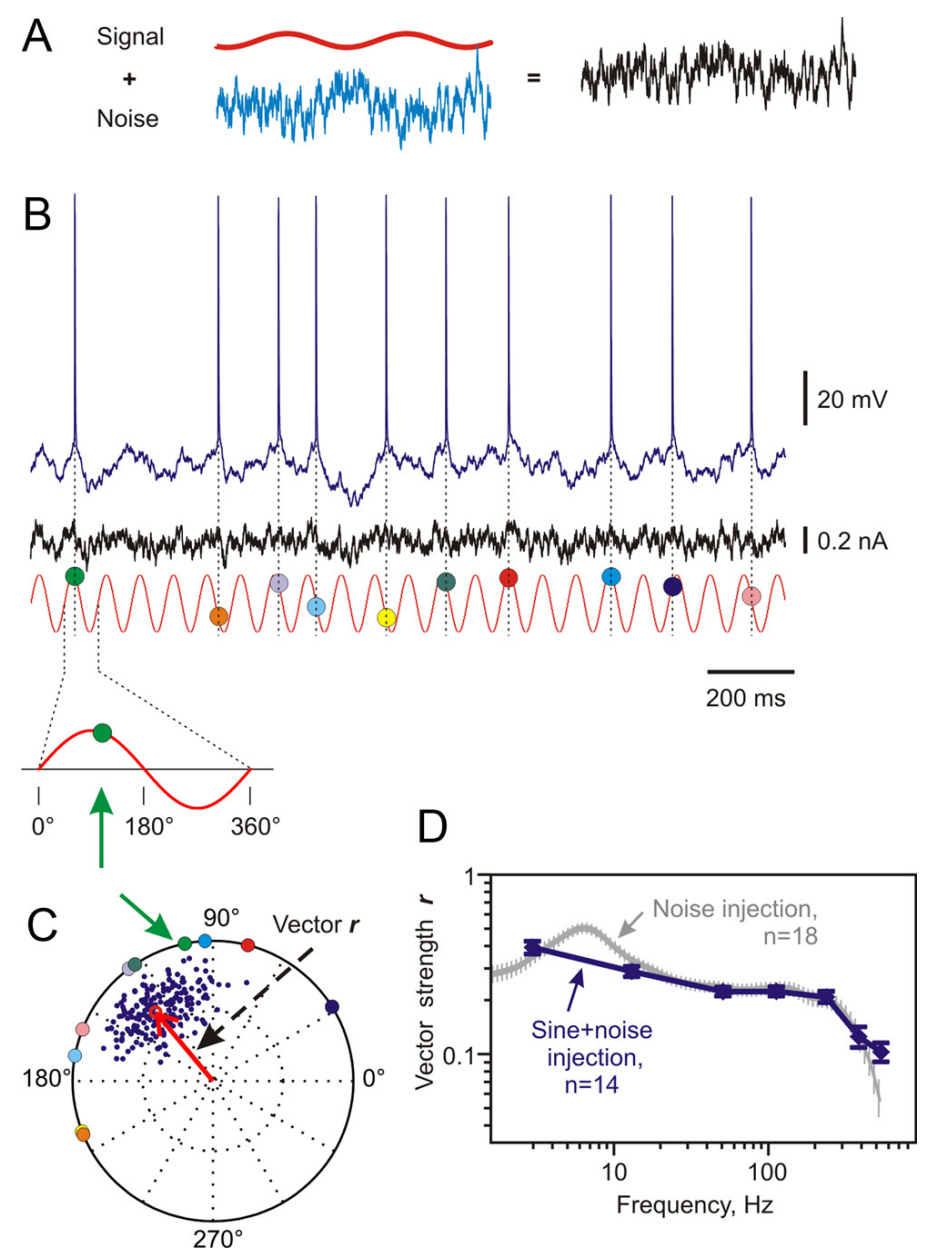

Fig 22. Vector strength method for calculating linear response functions A. A small sinusoidal signal is added to the background noise. B. Irregular firing patterns under the combined stimulus. For each spike time, it is mapped to one phase in the sinusoidal fluctuations. C. Each phase corresponds to a unit length vector in the complex plan. D. The average of all complex vectors normalized by the sinusoidal amplitude and the firing rate is the dynamic gain of related frequencies in the linear response function. The figure is from 9 .

functions is not different from zero. In figure B, the correlation time is $5 \mathrm{~ms}$, the sinusoidal amplitude is chosen to be $1 / 5$. To compare the performance of these two methods, the dynamic gain values at $10 \mathrm{~Hz}$ are aligned. We can see that the two linear response functions match each other up to $1000 \mathrm{~Hz}$. In figure $\mathrm{D}$, the correlation time is $50 \mathrm{~ms}$. The amplitude is chosen to be $1 / 2$. The dynamic gain values at $10 \mathrm{~Hz}$ are aligned. The two methods fit well at low frequencies. Due to the filtering artifact, the linear response function calculated with the Fourier transform method fluctuates in the high frequency regime.

In this section, I introduced two methods for calculating the linear response function. I also showed that these two methods' results fit with each other in a large frequency region. The Fourier transform method can provide more data points on the linear response function with less 

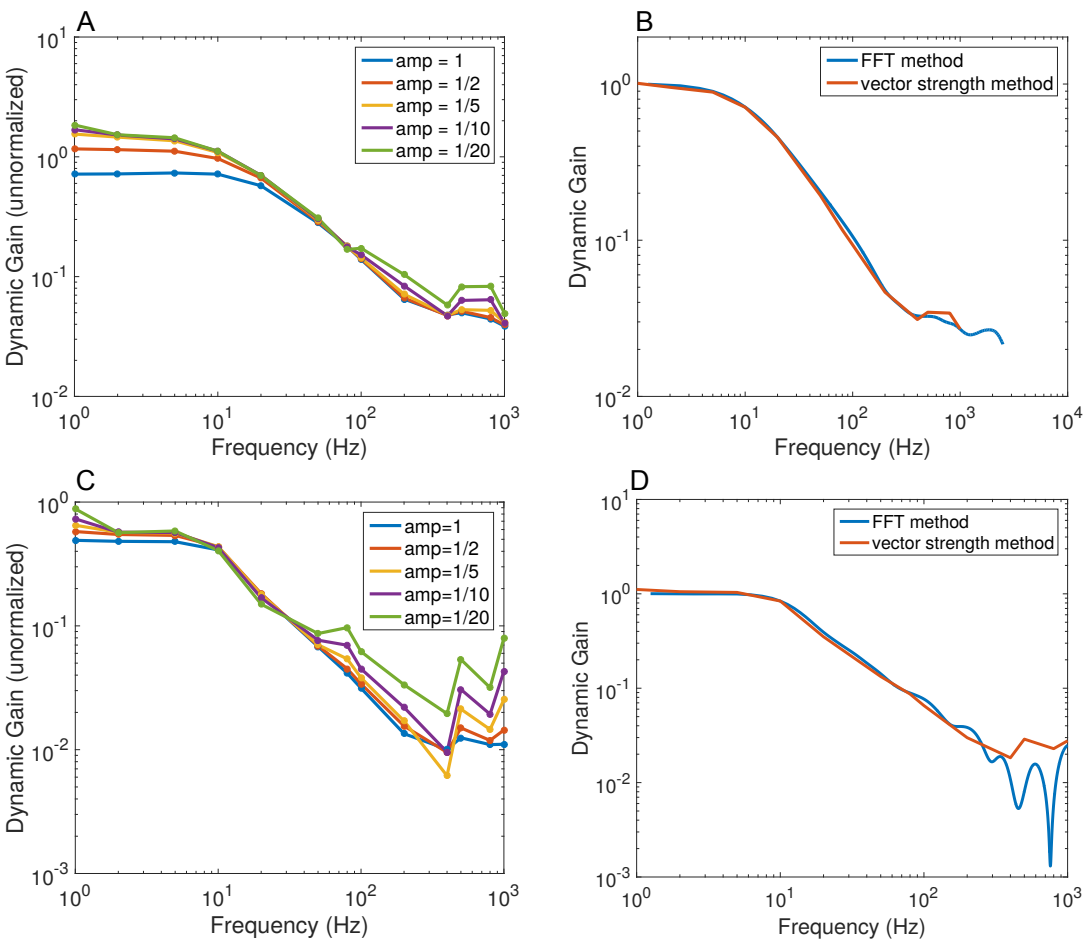

Fig 23. Calculation of the linear response function with the vector strength method. For $\mathrm{A}$ and $\mathrm{C}$, the linear response functions are calculated with the vector strength method. The dynamic gain is the absolute value of the averaged complex vectors divided by the sinusoidal amplitude. The linear response functions are not normalized with the first elements of the curve. For B and D, it is a comparison of the linear response curves of Brette's model calculated with the Fourier transform method and the vector strength method. Position of the sodium channels is $P_{N a}=20 \mu \mathrm{m}$. Correlation time of the stimulus is $5 \mathrm{~ms}$ in B and $50 \mathrm{~ms}$ in D. The sinusoidal amplitudes for all frequencies are $1 / 5$ in B and $1 / 2$ in D. The linear response function of the Fourier transform method is normalized. For comparison, in $\mathrm{B}$, the $20 \mathrm{~Hz}$ components of the vector strength method is aligned withe $20 \mathrm{~Hz}$ components of the Fourier transform method. In $\mathrm{D}$, the alignment frequency is $10 \mathrm{~Hz}$.

computation time. However, in the high frequency regime, the linear response functions are quite noisy. The vector strength methods requires more computation time. Also, it is necessary to examine whether the dynamic gain obtained with the given sinusoidal amplitude is in the linear regime. The linear response functions in the following chapters will be calculated with the Fourier transform method. In the next section, I will introduce the methods for estimating the confidence intervals of the linear response functions.

\subsection{Bootstrapping and Null Hypothesis Testing}

To test the accuracy of the linear response function calculation, I estimated the bootstrap confidence interval and tested the null hypothesis.

As mentioned above, each linear response function is calculated from the average of 400 pieces of STA. For bootstrapping, I performed random 
sampling with replacement over these 400 pieces of STA to calculate a new linear response function 1000 times. The upper bound and the lower bound of the confidence interval represent the range of the middle 95 percent of the 1000 curves.

For the null hypothesis test, I shuffled the spike times from each 20 seconds of simulations. Keeping the neighboring spikes intervals, we added a random number to all spike times, then the spike times are mod 20. In this way, the CVs of ISI are the same, so that the firing irregularities are the same. The shuffled spike times provide no information about the feature of the stimulus. For each shuffling, we obtained 400 new STAs and then one new linear response function. Repeating this procedure 500 times, we obtained the 95 percent upper bound from the 500 curves as the dynamic gain significance border. When the linear response curve are below this border, it cannot be distinguished from zero gain, which indicates the gain curve are not reliable any more. In Fig 24, the plateau of the null hypothesis curve in low frequency regime originates from the small random numbers used in shuffling. The increase of the null hypothesis curve in high frequency region is not affected by the shuffling method.
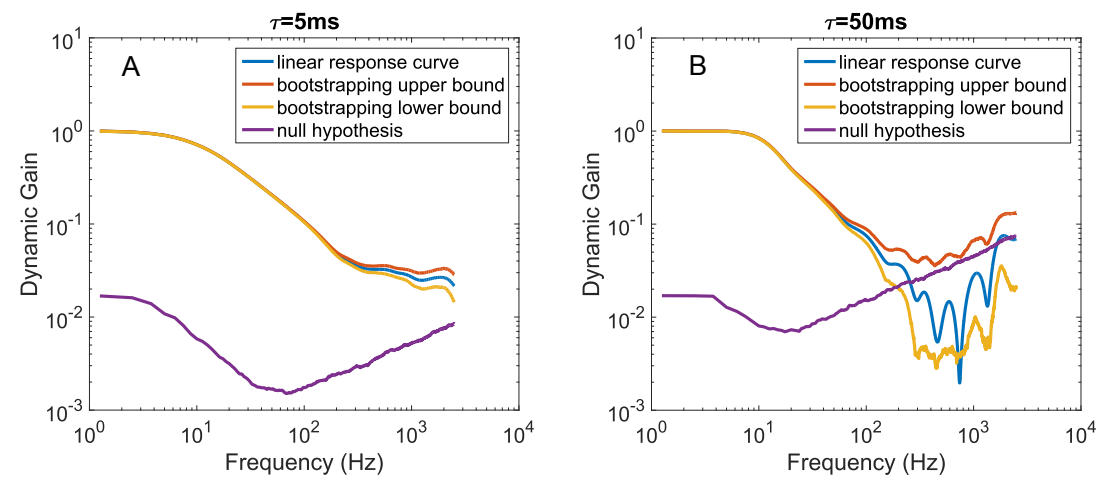

Fig 24. Bootstrapping confidence intervals and null hypothesis test. Based on Brette's model with $P_{N a}=20 \mu \mathrm{m}$, I provide an illustration of the bootstrapping confidence interval and null hypothesis curve. For the short correlation time of the stimulus, the confidence intervals are smaller than that of the large correlation time. The confidence interval becomes more fluctuated in the high frequency regime in B. The linear response curves outside the bootstrapping boundary is a result of the artifact of filtering. The null hypothesis curve is higher for large correlation time. The dynamic gain values after about $300 \mathrm{~Hz}$ are not significant.

Fig 24 gives an example based on Brette's model with $P_{N a}=20 \mu \mathrm{m}$. For short correlation time $5 \mathrm{~ms}$ in $\mathrm{A}$, we can see that the confidence interval are quite small up to $1000 \mathrm{~Hz}$. Also, the null hypothesis curve is well below the linear response curve. This implies that our simulation data are sufficient for a precise representation of the dynamic gain. The Gaussian filtering successfully smooth out the noise in the dynamic gain. As for the case of the large correlation time $50 \mathrm{~ms}$, the Gaussian filtering does not not exhibit a good enough performance. In the limit of large frequencies, there is a trend that dynamic gain and related confidence interval may rise again. This originates both from the reset by hand boundary effect and the noise decay rate smaller than the decay rate of power spectral density of the stimulus. The null hypothesis curve for the large correlation time is much higher than that with short correlation time. The linear response curve is 
below the null hypothesis curve around $300 \mathrm{~Hz}$. So in this case, the linear response function after that is not informative. In the following chapters, when analyzing the impact of different factors on the linear response curve, we will only compare frequency regions with a significant dynamic gain. Also, the bootstrapping confidence intervals are represented as shaded areas around the linear response curve.

\subsection{Evaluation of the AP Initiation Dynamics on Pop- ulation Encoding}

In this section, I will give an evaluation of the impact of AP initiation dynamics on population encoding. I will first show that previous quantification of the AP onset rapidness with a slope is inadequate to capture the encoding ability of the neuron model. The encoding ability is determined by both the AP initiation dynamics and the properties of stimulus. Then I will show that this complicated contribution can be described by the time delay between the spike initiation and the spike detection, which is a random variable. If there is no delay, the neuron model is similar to a LIF neuron. The distribution of time delay acts as a filter of the high bandwidth encoding.

In previous studies, when quantifying the AP onset rapidness of cortical neurons, people used the slope at a given voltage derivative in the phase plot 7]. In the simplified theta neuron, the onset rapidness is evaluated as the slope at the unstable fixed point in its I-V curve [43. In the subsequent studies, AP onset rapidness are proposed to be closely associated with the encoding ability of the cortical neurons 9 . One problem of the evaluation of the AP onset rapidness is that the choice of the slope is quite arbitrary which may not represent the key dynamic regions related to the encoding ability. For example in the theta neuron, the activation slope near the local minimum of the $\mathrm{I}-\mathrm{V}$ curve, $\mathrm{V}=0$, is much slower than that at the unstable fixed point. For a large mean but small std stimulus, we can expect that the voltage will be fluctuated around $\mathrm{V}=0$ for the most of the time. The spikes are triggered by random upward fluctuations. The spike generation time is mostly determined by the slow activation regime near $\mathrm{V}=0$. In this case, the slope at the unstable fixed point in the original I-V curve provides limited information about the AP onset rapidness. Similarly, if we change the stimulus into a small mean and large std one, the spikes are more likely to be generated by large upward fluctuations, which drive the voltage quick leaving the slow activation regime. The rapid AP generation dynamics will bring the voltage to the reset threshold, while the downward fluctuations from the stimulus are not able to counter this driving force. In this case, the slow activation dynamics near $\mathrm{V}=0$ play a less significant role. The activation dynamics in the larger voltages seems to be a better representation of the AP onset dynamics.

Fig 25 and Fig 26 are the simulation illustrations of the argument above. In Fig 25, I designed two models based on a theta model. The theta model is described as:

$$
\tau_{m} \frac{d V}{d t}=V^{2}-1 .
$$

The membrane time constant is $\tau_{m}=10 \mathrm{~ms}$. Once the voltage reaches the reset threshold $V=3$, it will be reset to -5 . The other two models have the same dynamics as the theta model when $V$ is smaller than 0 . When $V$ is larger than 0 , the neuron models are described by following equations 
separately:

$$
\begin{gathered}
\tau_{m} \frac{d V}{d t}=-0.2(V-1)(V-5) \\
\tau_{m} \frac{d V}{d t}=V^{1 / 2}-1
\end{gathered}
$$

The voltages will be reset to -5 once they reach $V=3$. We can see that all three models have the same local minimum $V=0$ and the same unstable fixed point $V=1$. For simplicity, we name the original theta model as theta1, the other two models as theta2 and theta3. At the unstable fixed point, theta1 model has the highest slope, while theta3 has the lowest slope. At the local minimum of the I-V curve, the slopes on the right hand side have the reversed relations. Following the evaluation criterion of previous work, we would expect that theta1 model should have the best encoding ability.

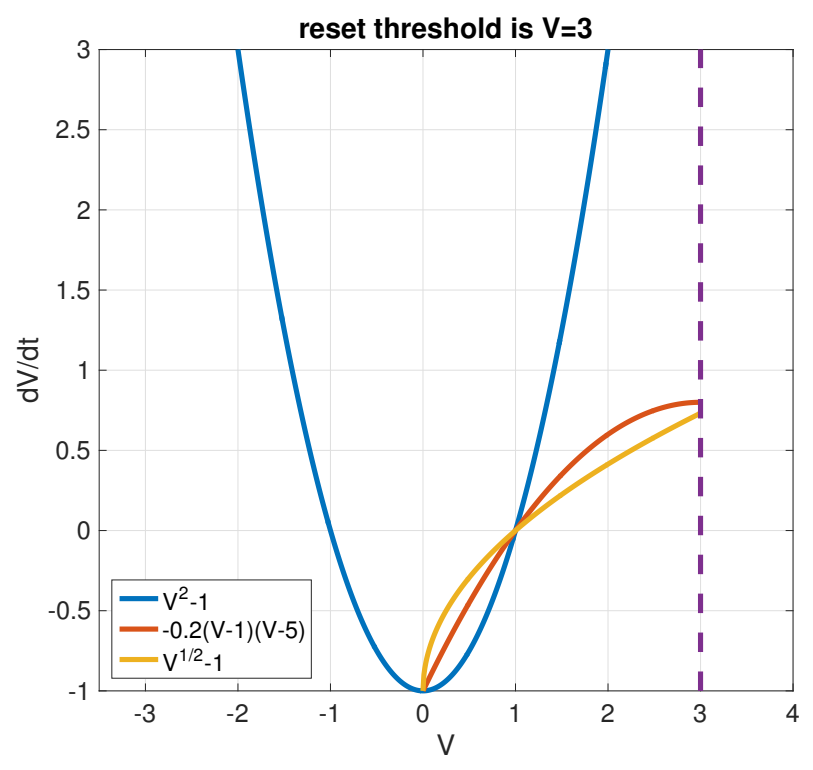

Fig 25. I-V curves of three neuron models. The first model is the theta neuron model shown as the blue curve. The voltage will be reset to -5 once it reaches the boundary $\mathrm{V}=3$. For the second and the third model, they have the same dynamics as the first model before $\mathrm{V}=0$. The dynamics after $\mathrm{V}=0$ are given in related equations. The boundary voltage and reset voltage are the same as those in the first model.

Fig 26 provides the linear response curves of three neuron models under two types of stimuli. Here in the simulation, I fixed the firing rate to be $5 \mathrm{~Hz}$. In figure A, the mean of the stimulus is fixed to be 0.95 . In figure B, the mean of the stimulus is fixed to be 0 . To calculate the linear response curve, I used $10 \%$ of the spike numbers as that in Brette's model introduced above. I generated 400 pieces of STA. For each STA, it is generated with 500 spikes. The bootstrapping are the same as that of the Brette's model. The null hypothesis curve is calculated with 100 trials of random shuffling. These three neuron models have simpler dynamics than Brette's model, so $10 \%$ data can still provide a quite precise result. The bootstrapping 
confidence intervals are on the scale of the line width. The thin lines below the linear response curves are the null hypothesis curves.

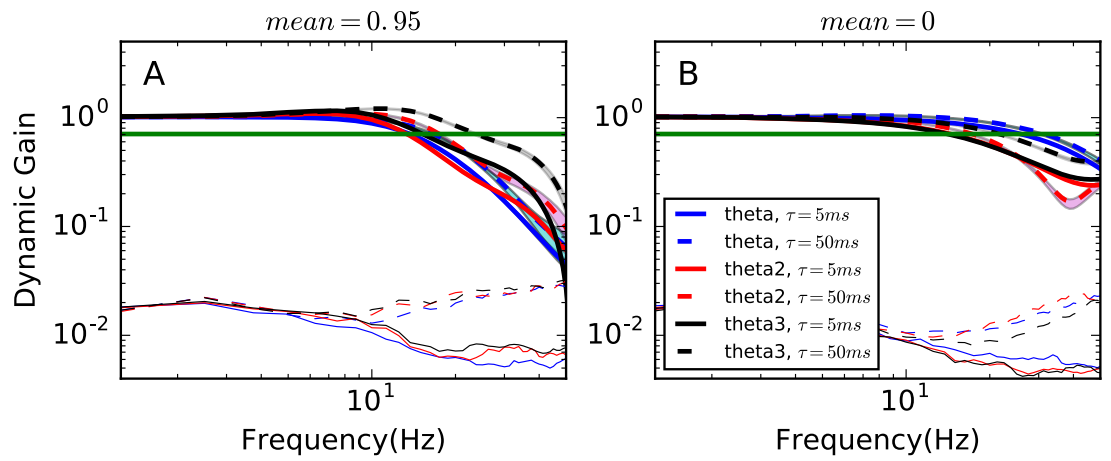

Fig 26. Linear response functions of three neuron models with different types of stimuli. The linear response functions for three models are in blue, red and black separately. The continuous lines are for the stimuli with correlation time $5 \mathrm{~ms}$. The dash lines are for the stimuli with correlation time $50 \mathrm{~ms}$. The thinner lines below are the null hypothesis curves for related linear response curves. In figure A, the mean of the stimuli are fixed to be 0.95 . The std of the stimuli are tuned to reproduce $5 \mathrm{~Hz}$ firing rates. The third model has the best encoding ability. In figure $\mathrm{B}$, the mean of the stimuli are fixed to be 0 . The firing rate is $5 \mathrm{~Hz}$. With large stimuli fluctuations, the first model has the best encoding ability.

In Fig $26 \mathrm{~A}$, the mean of stimuli are fixed to be 0.95 . The std of stimuli are much smaller. We can see that all three models have a low cutoff frequency in their linear response curves. This is due to the slow AP onset as a whole. However, we can still compare the encoding performances across different models. For the third model, the slope at $V=0$ from the right hand side is infinity. The voltage derivative will immediately reach a relatively high value once $V>0$ compared to the other two models. This helps to bring the voltage to generate a spike rather than fluctuated in the low voltage regime. Note here the key element for encoding is the high value of voltage derivative rather than the slope at one particular point. An sufficient amount of increase of voltage derivative in a short voltage interval usually implies high slope existed in this voltage interval. However a high slope at one given point doesn't imply high voltage derivative. In this sense, what really matters is the "average AP initiation speed" in a sufficiently large voltage interval, rather than "how fast the AP initiation speed changes". This idea is supported by the Fig 26B. When the mean of stimuli are fixed to be 0 , the std of stimuli are much larger. With large voltage fluctuations, the spikes are more likely to be triggered by the large voltage kick originated from the stimuli, rather than brought up by the AP initiation dynamics near $V=0$. The large voltage kick brings the voltage to higher values. The voltage derivative values at related regime matters more for the AP onset rapidness. So we can see from the figure B that the first theta model shows a slightly better encoding ability. The results above support our assertion that quantifying the AP onset rapidness as the slope at a given point in the I-V curve is inadequate for quantifying encoding ability. For the more general case in the experiments, without the knowledge of neuron equations, describing the AP onset with the slope at one point in the phase plot has the exactly the same problem. 
Up till now, we have already known that the encoding ability is a complicated properties determined by the stimulus property and AP initiation dynamics. A slope is not sufficient to describe this property. So the question becomes: is there a parameter to capture the encoding ability of a neuron model? In the following part of this section, I will show that this parameter is the time delay between the spike initiation and the spike detection.

It has been known that the LIF neuron has an extremely good encoding ability [39. In its I-V curve, the reset threshold is set at the voltage having the global minimum of voltage derivative. The neuron model doesn't have the AP generation dynamics. We can assume that AP initiation speed is infinite. The spike detection time is exactly the spike initiation time. However, if the reset voltage is not at the minimum of the voltage derivative, for example, the theta neuron, the spikes are generated with a saddle node bifurcation when injecting a constant input to the neuron. Then it takes some time for the voltage to reach the reset threshold. In the examples above, the theta neuron model fails to reproduce the high bandwidth encoding. However, it can be expected that if we shift the reset threshold towards the minimum of the voltage derivative voltage, the encoding ability would be similar to that of a LIF-like neuron.

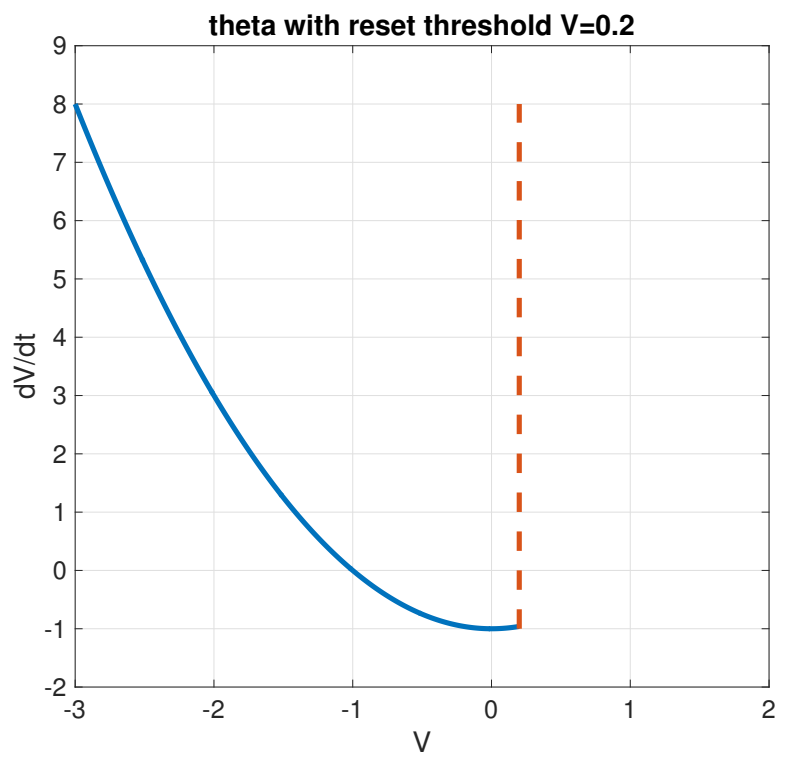

Fig 27. Theta neuron model with reset threshold at $V=0.2$. The time constant of the neuron model is $1 \mathrm{~ms}$. Once the voltage reaches the reset threshold, it will be reset to -5 .

Fig 28 is a neuron model designed based on this idea. The neuron equation is:

$$
\tau_{m} \frac{d V}{d t}=V^{2}-1
$$

The reset threshold is set to $V=0.2$. Once the voltage reaches this value, it will be reset to $V=-5$. Here the time constant of the neuron model is set to be $1 \mathrm{~ms}$. We denote this neuron model as theta4. Based on the neuron dynamics, we can see that it is quite close to the LIF-like neuron models, except that the reset threshold is slightly shifted from $V=0$. One 
may expect that the encoding ability of this model should be similar to that of the LIF neuron. However, with the simulation knowledge of three theta neurons above, we know that the encoding should be dependent on the properties of stimulus. Fig 28 shows the linear response functions generated with two types of stimuli. For large std stimuli, the linear response functions share the similar properties of those of the LIF model. Increasing the correlation time of the stimulus enhances the dynamic gain in the high frequency regime. In contrast, the linear response functions generated with the small std stimuli have much lower cutoff frequencies. The dynamic gain values are also insensitive to the correlation time of the stimuli.
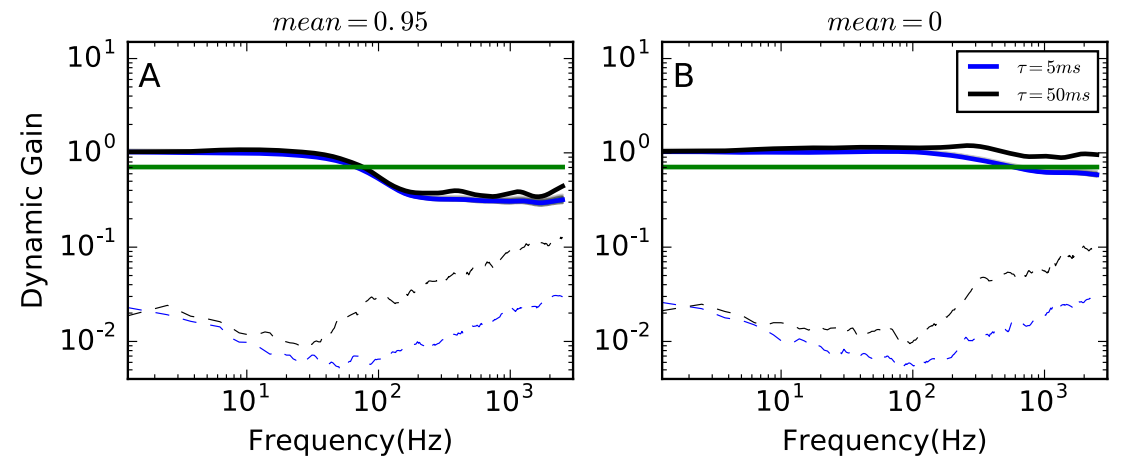

Fig 28. Linear response curves of the theta neuron with reset threshold $V=0.2$. The denotations are the same as Fig 26. The firing rates of all conditions are tuned to be $5 \mathrm{~Hz}$. In figure $\mathrm{A}$, with a large mean and a small std stimulus, the linear response function shows a lower cutoff frequency compared to those generated with a zero mean and a large std stimulus in B. Also the dynamic gain in the high frequency regime is insensitive to the correlation time of the input in figure A.

One intuitive explanation for the different performances of the linear response functions can be deduced based on the spike time detection. For the case of large std stimulus, the spikes are triggered by the kick of the stimulus. The AP generation dynamics play a limited role. If we inject the same stimulus to the theta neuron model with a reset threshold at $V=0$, the spike detection time should be almost in the same time bins as that with reset threshold at $V=0.2$. The STAs obtained for calculating the linear response functions are almost the same. However, if the stimuli have a large mean and a small std, the extremely slow AP generation dynamics near $V=0.2$ matters for the spike time detection. In the case of reset voltage at $V=0$, a spike is detected when the stimuli drives the voltage to touch $V=0$. But for the case of reset threshold $V=0.2$, it still takes another period of "random walk" to make the spike time reach the new reset threshold. Since the std is small, each step of the random walk is relatively small. The expected time to reach $V=0.2$ is longer. So compared with the spike time obtained in $V=0$, the spike times in this case first may have a few missing because of the spike failure. Here the spike failure is defined as the voltage fluctuates across 0 without touching the reset threshold. Second, the existing spike times have different degrees of delays compared with the LIF like model. So the STA is a distorted version compared to the "perfect" one in the LIF like model.

Fig 29 shows the probability histograms of spike time detection delays for two types of stimuli. For each type of stimulus, I simulated the neuron 

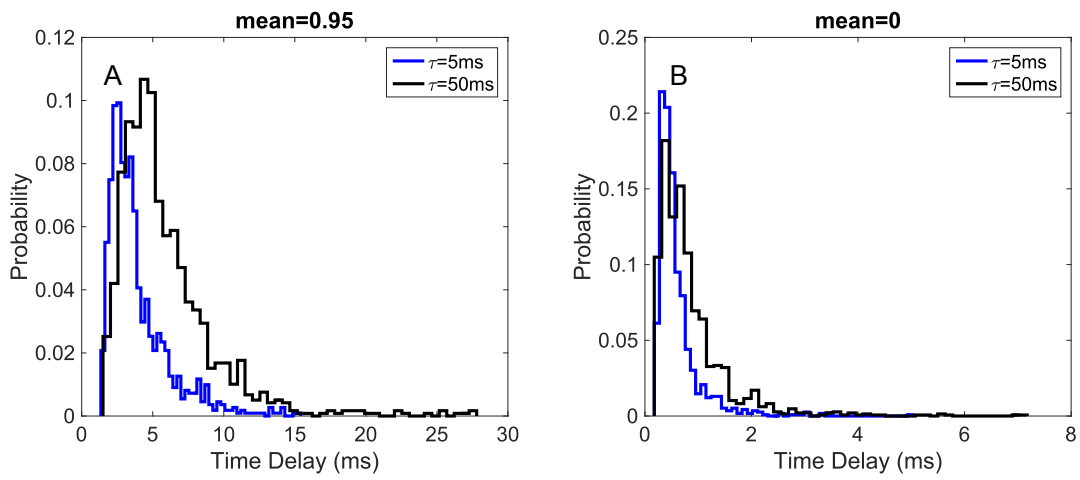

Fig 29. Probability histograms of time delay between spike initiation and spike detection. A: For the large mean small std stimulus, the probability histograms of the time delay have a larger range. B: In comparison, for the zero mean large std stimulus, the probability histograms of the time delay are more centralized around small values.

model for 200 seconds. The time points that the voltage reaches the reset threshold is taken as the spike detection time. The last time point that the voltage passes $V=0$ from below before reaching the reset threshold is chosen as the spike initiation time. The time delay from the spike initiation to the spike detection is a random variable that describes the contribution of AP generation dynamics and stimulus properties on the encoding. If the probability distribution of this random variable has a high std, it implies that the neuron model is highly unreliable of tracking the spike initiation. On the other hand, if the probability distribution is a delta function, it implies that the neuron model has a perfect representation of spike initiation. Once it detects the spike initiation time, it will spend a fixed time interval of time to report this time point. For the LIF-like neuron, this fixed time interval is zero. For more general case, it means the neuron generate a spike of which the spike shape is not affected by the stimulus. From Fig 29 we can see that when the stimulus has a zero mean and large std, the time delay distribution is highly centralized around $1 \mathrm{~ms}$. The probability distribution decays quickly when the time delay gets larger. A larger correlation time of the stimulus actually broadens this distribution. This implies that the sensitivity of the dynamic gain to the correlation time is mainly from the contribution of the LIF like model. A finite time spike generation dynamics reduces the sensitivity. For those neuron models that fails to observe the contribution of the correlation time in the linear response functions, it is because the time delay distribution is too broad. In figure A, it shows the time delay distribution of the stimulus having a large mean and a small std. The distributions have a larger std. The decay of the distribution tail is slower.

Following the results on the simplified 1-D neuron model, we can ask the following two questions. How to generalize this observation to the more complicated conductance-based models or even experiments? Is it possible to separate out the effect of AP initiation dynamics from the linear response functions, so that the final linear response function can be represented as a multiplication of a LIF like linear response function with a low pass-filter? For the first question, it is quite intuitive to solve. In the theoretical modeling, the neuron models are classified as type I and type II based on the type of bifurcations at the spike initiation. We call 
the voltage where the bifurcations happen as the rheobase voltage, which is the term spike initiation voltage used above. When the input is stochastic, even if the stimulus has pushed voltage across the rheobase voltage, it is still possible to bring down the voltage without generating a spike. So we will take the last time point that the voltage passes the rheobase voltage in the positive direction before the spike as the spike initiation point. To track this time point, we first need a high enough voltage to locate the existence of a spike, then we can find out the nearest time point crossing the rheobase voltage. This criterion can also be used in the experiment. By injecting constant inputs into the neuron, we can find the rheobase voltage. This also applies for the complicated neuron models with no explicit expression of the rheobase voltage. As for the high enough voltage to locate the spikes, the criterion I introduced in Brette's model is the voltage that has the maximum voltage derivative during a spike. With this choice of the spike detection voltage, we only take the time delay before this voltage into the calculation of the linear response function. The neuron model we are examining can be approximated as having a reset threshold at the spike detection voltage.

Fig 30 is an illustration of this idea. Here I used the Wang-Buzsaki model, denoted as WB model below. WB model is a type I conductancebased neuron model. We can numerically determine the rheobase voltage at $-60 \mathrm{mV}$. Injecting a constant input to make it fire at $5 \mathrm{~Hz}$, the voltage that has the maximum voltage derivative is $-9 \mathrm{mV}$. Fig 30 A marks out the time points that voltage reaches $-60 \mathrm{mV}$ and $-9 \mathrm{mV}$ during spike initiations. If we take the spike times found with $-60 \mathrm{mV}$, the neuron model is similar to a LIF neuron. All the time delays caused by AP generation dynamics are abandoned. The STA calculated in this condition has a large amplitude. The peak of the STA is exactly at the center of the STA time interval. If we take the spike time found with $-9 \mathrm{mV}$, the spike times have different delays compared to those found by $-60 \mathrm{mV}$. The pieces of stimuli centered at the new spike times are shifted to the right in the time domain compared with the old one. The peak of the related STA are shifted to the left. In Fig $30 \mathrm{~B}$, the STA calculated with the spike times at $-9 \mathrm{mV}$ are flattened and shifted to the left. Here the spike numbers used for STA is $10 \%$ of that used in Brette's model. The correlation time of the stimulus is $50 \mathrm{~ms}$.

It can be expected that if we change the spike detection voltage from $-60 \mathrm{mV}$ to $-9 \mathrm{mV}$, the LIF like STA will converge to the flattened STA. The corresponding linear response curves will converge from high cutoff frequency to low cutoff frequency. Also the spike time delay distribution will change from a delta function to a distribution with broad range. Fig 31 is an illustration of the impact of spike detection voltage. The linear response function calculated with the spike time voltage with maximum voltage derivative, which is $-9 \mathrm{mV}$, are generated with the same amount of spikes as that in Brette's model. The other three linear response curves are generated with $10 \%$ of the data. Here I omit the null hypothesis curves.

When the spike time voltage is at the spike initiation voltage, the linear response functions are similar to that of the LIF model. In the large frequency limit, the dynamic gain values become constant. Moving the spike time voltage towards $-9 m V$, which has the maximum voltage derivative, the dynamic gain converges to related values. During the convergence, the dynamic gain in the high frequency regime remains the feature of becoming a constant. Based on this observation, we can expect that the linear response function with spike voltage at $-9 m V$ decays with a slope of -1 in the intermediate frequencies in $\log -\log$ scale. The dynamic gain in this 

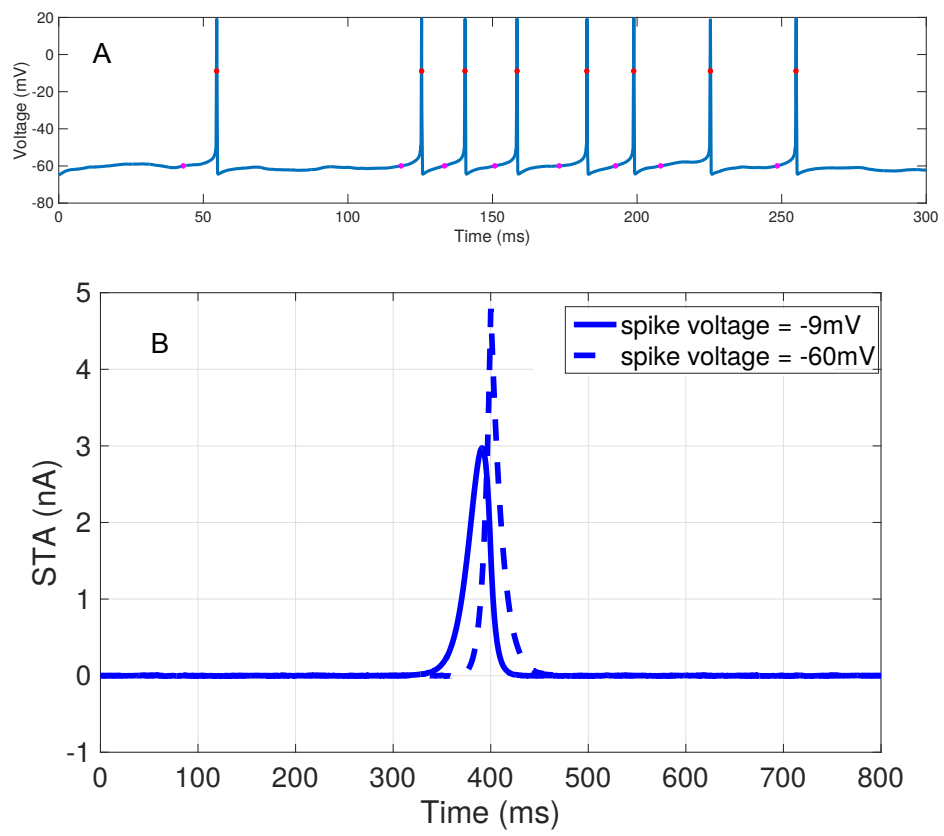

Fig 30. Comparison of the STA calculated with the spike voltage at $-9 \mathrm{mV}$ and spike voltage at $-60 \mathrm{mV}$. A: an illustration of the spiking voltage traces of Wang-Buzsaki model. The correlation time of the stimulus is $50 \mathrm{~ms}$. The red dots in the voltage trace indicate the spike times when the spike time detection voltage is $-9 \mathrm{mV}$ and $-60 \mathrm{mV}$ separately. B: a comparison of the STA calculated based on these two spike time voltages. At $-60 \mathrm{mV}$, there is no spike time detection delay, the STA has a larger amplitude. The peak of the STA is at the center of the time interval. At $-9 \mathrm{mV}$, the STA is filtered by the random spike detection delay. The peak of the STA is shifted to the left.
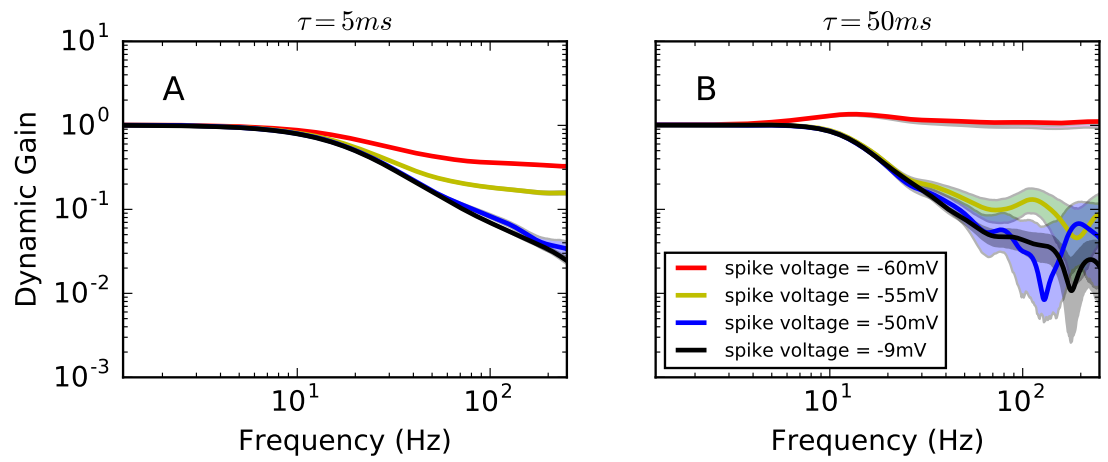

Fig 31. Comparison of the linear response functions calculated with different spike voltages. The mean and the std of the stimulus are tuned to reproduce $5 \mathrm{~Hz}$ firing rate. The CV of ISI is around 0.85 . The linear response functions are similar to those of LIF models when the spike detection voltage is near the spike initiation voltage. The dynamic gain values in low frequencies converge more quickly to those of the spike voltage at $-9 m V$ when the correlation time of the stimulus is larger.

range is determined by the exponential $\mathrm{AP}$ activation function. In the limit 
of large frequencies, the linear response function becomes flat, which is the boundary effect of finite spiking voltage. For the large correlation time of the stimulus, the dynamic gain in the low frequency regime converges more quickly. For both correlation times, the uncertainty introduced by the spike detection delay is mainly originated from the $10 \mathrm{mV}$ from $-60 \mathrm{mV}$ to $-50 \mathrm{mV}$. The linear response functions generated with spike voltage at $-50 \mathrm{mV}$ is already a close approximation of those with spike voltage at $-9 m \mathrm{~V}$. This implies that the spike time detection delay distribution is already close to be stabilized.

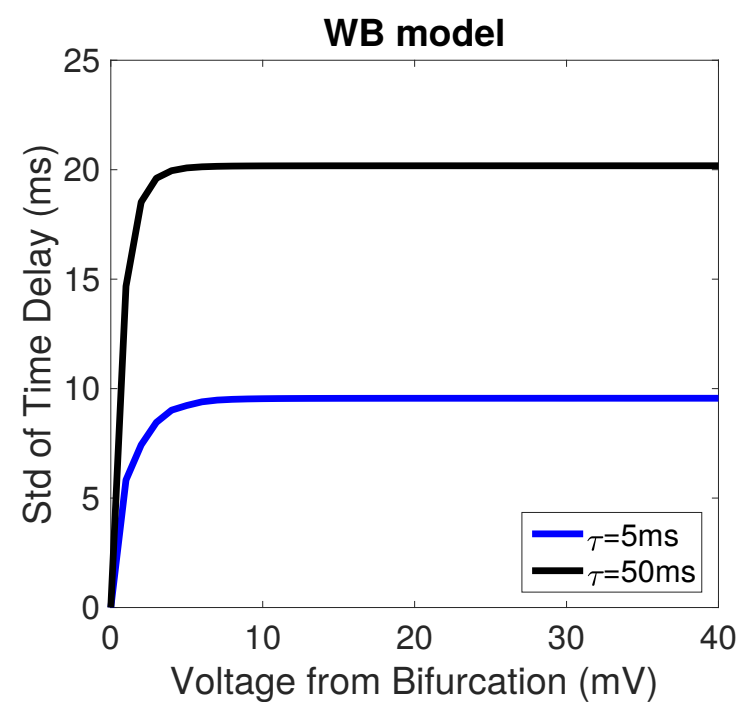

Fig 32. The std of the spike detection delay across different spike voltages. 200 seconds of spiking voltages are generated for spike time detection. The bifurcation voltage is at $-60 \mathrm{mV}$. For different spike detection voltages, the std of the spike detection delay is calculated as an estimation of the uncertainty introduced by the dynamics between the bifurcation voltage and the spike detection voltage. Here the figure plots the std over the voltage difference in between.

To quantify how much uncertainty is introduced by each part of the AP generation dynamics, the std of the spike detection delay is plotted over the voltage difference between the spike initiation voltage and the spike detection voltage shown in Fig 32, 200 seconds of spiking voltages was simulated for the estimation of std. With the exponential AP generation dynamics in WB model, the slow AP generation dynamics near the spike initiation voltage brings the majority of the uncertainty in spike time detection. The increase of std in each voltage step describes the impact of this part of AP generation dynamics on encoding. When the voltage difference from the spike initiation is $10 \mathrm{mV}$, the std of time delay has reached its convergence, which fits with the observation in the linear response function. Another observation is that a longer correlation time of the stimulus introduces more uncertainty. From the view of simulation, with a given time step, large correlation time implies less stimulus change in each time step. A small stimulus kick elicits to a limited AP generation driving force, which makes it easier to bring down the voltage again in the next time step. In contrast, with a shorter correlation, it is less likely to bring back the voltage after a large voltage kick. As a result, the voltage may linger in the low voltage 
regime for a longer time when the correlation time is large.

I have shown that the impact of AP initiation dynamics and stimulus properties on encoding is to introduce random time delay between the spike initiation time and the spike detection time. Ideally, if there is no time delay, the linear response functions are similar to those of LIF models. In reality, the random time delay undermines the encoding ability in the high frequency regime. The linear response functions generated with the stimuli having long correlation times are affected more. The contribution of each part of the AP initiation dynamics on the randomness of time delay can be described by the increase of std of random time delay. For exponential AP activation dynamics in WB model, the majority of the randomness is caused by the $10 \mathrm{mV}$ dynamics after the bifurcation voltage. Now it comes to address the question of separating the low pass filtering effect of spike detection delay on the linear response function.

Denote the spike initiation times in one trial of simulation as $t_{1}, t_{2}, \ldots, t_{N}$. The corresponding spike detection delays are denoted as $x_{1}, x_{2}, \ldots, x_{N}$. The pieces of stimuli centered at the spike initiation times are $s\left(t_{1}\right), s\left(t_{2}\right), \ldots, s\left(t_{N}\right)$. Each piece of stimulus $s\left(t_{i}\right)$ is of length $T$. Similarly, the pieces of stimuli centered at the spike detection times are $s\left(t_{1}+x_{1}\right), s\left(t_{2}+x_{2}\right), \ldots, s\left(t_{N}+x_{N}\right)$. The LIF like STA is an average of all $s\left(t_{i}\right)$ aligned together, represented as: $S T A_{L I F}(t)=\frac{1}{N} \sum_{i=1}^{N} s\left(t_{i}\right)$. Here $t$ takes the range of $\left[-\frac{T}{2}, \frac{T}{2}\right]$. The STA filtered by the spike detection delay is $S T A_{\text {filtered }}(t)=\frac{1}{N} \sum_{i=1}^{N} s\left(t_{i}+x_{i}\right)$. With the given AP initiation dynamics and stimulus, the spike detection delay obeys the distribution $P(x)$. If the delay is a fixed number $x^{*}$ independent of other factors, then $S T A_{\text {filtered }}(0)=S T A_{L I F}\left(x^{*}\right)$. The filtered STA is a shift of LIF like STA to the left. More generally, for all the stimuli $s\left(t_{k_{x}}\right)$ that has a spike detection delay of $x$, denote their average as $S T A_{(x)}(t)$. Then:

$$
S T A_{\text {filteted }}(t)=\int_{0}^{\infty} S T A_{(x)}(t+x) P(x) d x
$$

With the current model and stimulus properties, a few observations can be made as below. First, although in theory the range of $x$ can go to infinity, in the neuron modeling it usually takes of the value of a few ms to a few tenth ms. So the distribution of $x$ is mainly concentrated in a relatively short range. Second, it can be expected that the STA of large time delay have less amplitude than that of short time delay. If it doesn't require too much current to have a firing rate difference, and the firing patterns doesn't consist of some pieces with too low firing rate and some other pieces with too high firing rate, then the STA for different time delay should be similar. This is equivalent to require that the F-I curve of the neuron model doesn't have a too low slope. Also the firing patterns are not consist of some pieces of too low firing and some pieces of too high firing, which implies a high CV or a change of stimulus properties during the simulation. For the simulation on WB model, the conditions above seem to be satisfied, so we would expect no too big differences between different $S T A_{x}(t)$. Then we can assume for the following approximation:

$$
S T A_{\text {filtered }}(t) \approx \int_{0}^{\infty} S T A_{L I F}(t+x) P(x) d x
$$

To examine this assumption, I compared the STA obtained with the spike detection time and the STA obtained with the spike initiation time and filtered with the detection delay, as shown in Fig 33. 

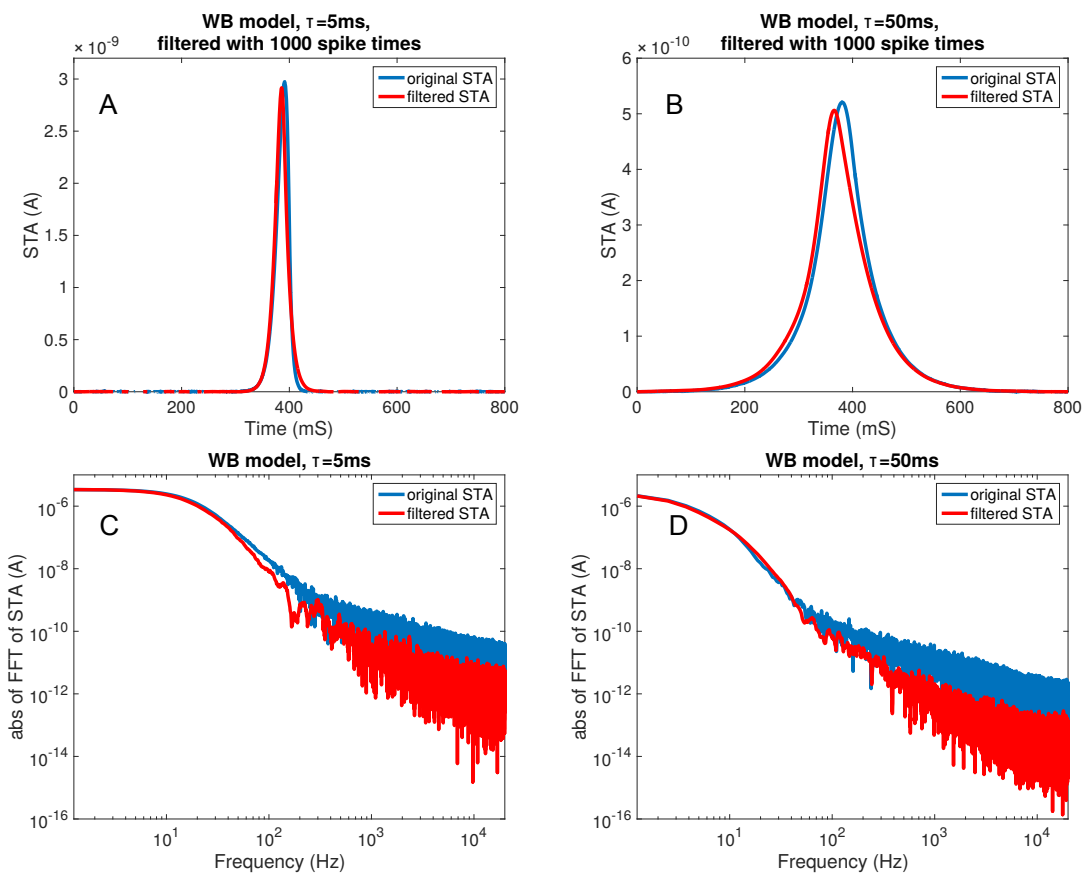

Fig 33. Comparison of the STA of WB model and its approximation as the filtering of a LIF like STA. The STA curves from the linear response functions in Fig 31. The distributions of the spike detection delay are approximated with 1000 sampling values from the simulation. For both correlation times of the stimuli, the filtered STA fits relatively good with the original STA. Comparing the STA in the frequency domain, it can be seen that the major difference of the two is in the high frequency regime.

For each type of stimulus, we obtained 1000 spike time delays as an approximation of the true distribution of $\tau$. The STA obtained with the spike initiation time is filtered as $\frac{1}{N} \sum_{i=1}^{N} S T A_{L I F}\left(t+\tau_{i}\right)$. The filtered STA is a relatively good approximation of the original one, which captures the shift of the maximum of STA from the middle of the time interval. The difference between the two curves are mainly from the amplitudes of the STA having different time delays. Those STAs with shorter delays should have slightly larger amplitudes, which make the original STA have a larger amplitude and a peak near the center of the interval. For further comparison, I examined the STA in the frequency domain. In the low frequency regime, the frequency components from two STA fit with each other. In the high frequency regime, the filtered STA shows less amplitude. However, as we have show in the simulation methods above, the high frequency components are dominated by the noise, which provides limited information about the true frequency components values.

I also examined the linear response curves directly for the approximation. The final gain curve is the Fourier transform components of STA divided by the power spectral density of the stimulus. The differences in the frequency components are amplified by the power spectral density. For the large correlation time, the power spectral density decays faster with the frequency compared to that of the short correlation time. That's why the frequency components of the STA seem to fit better, but the gain curves 

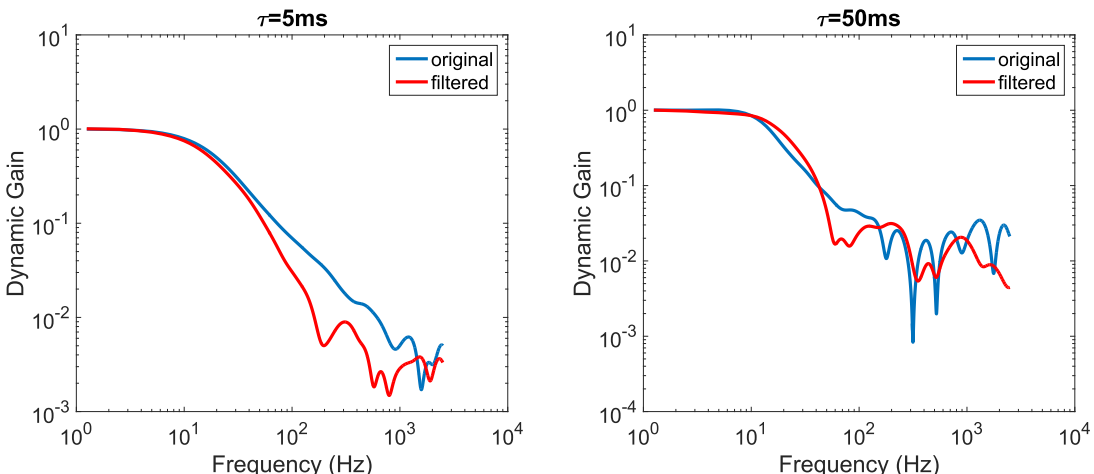

Fig 34. Comparison of the linear response curves of WB model and their approximations as the filtering of a LIF like STA. For both linear response curves, the differences in the frequency components of STA are amplified by the power spectral density of the stimulus.

have larger differences. Note here that for the filtered STA, the phase shift of the frequency components are more fluctuated than the original ones. When smoothing out the noise in the frequency components, it is necessary to check where to cut the STA for the Fourier transform. To find the optimized cut point, I calculated the std of the first 200 to 300 phase shift terms, and took the cut point that has the least std of the phase shift terms. The number of the phase shift chosen for calculating std depends on the correlation time of the stimulus. For short correlation times, the phase shift is less noisy, it is eligible to choose a larger range of phase shift values. For long correlation times, the phase shift becomes noisy quickly with the increase of frequency. So less terms can be chosen for std.

As a summary, here I proposed an approximation to separate the impact of AP initiation dynamics and stimulus properties on spike time detection delay from the linear response functions of the neuron model, such that it can be represented as LIF like linear response functions and low pass filters. The approximation provides a relatively good performance for the low frequency components of the STA. The differences in the frequency components are amplified by the power spectral density shown in the linear response curves. For further refinement of the approximation, the adjustment of the amplitude of the STA with different time delay should be considered. 


\section{The Impact of AP Initiation Site Location on Fast Population Encoding}

In this chapter, I will examine the axonal hypothesis proposed by Brette [48]. I will show that axonal AP initiation on its own is not sufficient to generate an ultrafast response and reproduce high bandwidth encoding observed in cortical neuron populations. The linear response functions in the high frequency regime are furthermore insensitive to the correlation times of inputs, which also contradicts previous experiments. Tuning the activation slope of sodium current, I will examine the impact of voltage sensitivity of sodium channels on population encoding. When high voltage sensitivity is introduced, the two disagreements are resolved. I will give an explanation for the dissociation of onset rapidness and the ultrafast response. The results above will show that high onset rapidness of action potential initiation is not sufficient for the ultrafast response. In the last section of this chapter, I will introduce a simplified description of the multi-compartment model with threshold sodium channel gating. The analytic linear response formula highlights the roles of different parameters on encoding ability.

\subsection{The Impact of AP initiation Site on Population Encoding}

According to the axonal hypothesis, spikes are initiated in the axon separated from the soma 59, 90. Increasing the separation distance of the AP initiation site makes the sodium activation dynamics seen from the soma more instantaneous. The spiking voltage at the soma has a sharp onset, which leads to fast population encoding. One supporting evidence of this proposal is the relation between AP initiation site and lateral current entering soma. Fixing somatic voltage at different values, when the separation of AP initiation site is large enough, the lateral current has a step-like change. The sodium activation ratio also has a discontinuity [48].

The axonal hypothesis highlights the role of neuron morphology on population encoding in cortical neurons. The detailed structure of cortical neurons are neglected in traditional simplified single-compartment models. The axonal hypothesis provides a more complete description of the neuronal dynamics. Recently more studies have been working on the contribution of different neuronal parts to AP generation dynamics and population encoding 49,52. Following the path of axonal hypothesis, there are two aspects remained for further examination. One is in the dynamic spiking condition, how the sodium activation dynamics seen from the soma change with the AP initiation site. It is necessary to check that whether the instantaneous step-like change observed in the stationary condition still holds in the dynamic spiking condition. The second aspect is the direct calculation of the linear response functions to study the impact of AP initiation site on dynamic gain. For more detailed illustration of the axonal hypothesis, it is given in the introduction. The neuron model setup and simulation methods are given in the previous method chapter.

Fig 35 examines the sodium activation dynamics for different positions of sodium channels $P_{N a}$. Fig 35 A shows the activation ratio of sodium channels $m$ as a function of axonal voltage $V_{a}$ recorded at the AP initiation site. The activation function can be described as $m\left(V_{a}\right)=1 /\left(1+\exp \left(\left(V_{m}-V_{a}\right) / k_{a}\right)\right)$. For standard sodium activation dynamics, $k_{a}$ is set to $6 \mathrm{mV}$. Fig $35 \mathrm{~B}$ compares original activation curve with representations of $m$ as the functions 
of somatic voltage recorded at the center of soma given three different AP initiation sites. Injecting a constant input which generates $5 \mathrm{~Hz}$ firing rate in each model, I recorded the somatic voltage $V_{s}$ and the sodium activation variable $m$ at the same time. As the positions of sodium channels move further away from the soma, the activation curves rise up more rapidly in lower voltage regime. This indicates that the observation in stationary condition is also valid in dynamic spiking condition. Here we remove the reset threshold in the simulation so that more complete curves can be plot. The constant input that generates $5 \mathrm{~Hz}$ firing rate is determined with the models having their reset thresholds.
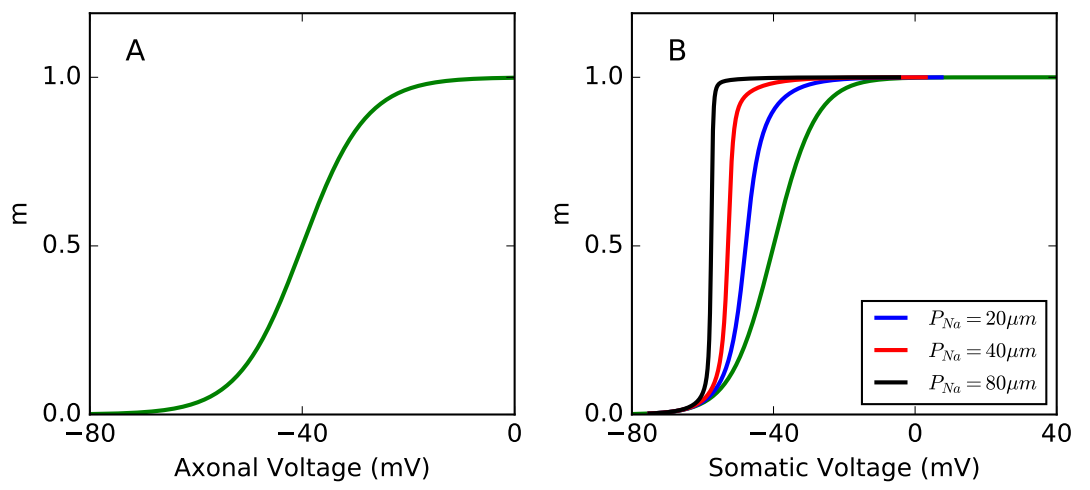

Fig 35. Apparent sodium activation curves versus axonal and somatic voltage. A: Standard sodium activation curve represented as the function of axonal voltage. Activation parameter is $k_{a}=6 \mathrm{mV}$. B: Represented as the function of somatic voltage, the sodium activation variable appears more voltage sensitive as the initiation site is further away from the soma. The green curve is the activation curve from A for comparison.

From the perspective of spike shapes and their phase plots, a larger separation of AP initiation site makes the spikes sharper seen at the soma. Injecting a constant input to drive the neuron model firing at about $5 \mathrm{~Hz}, \mathrm{I}$ plot the spikes seen at the soma in Fig $36 \mathrm{~B}$. Since the spike generation is in the axon, somatic voltage has a time delay and rises later than axonal voltage. However, somatic voltage is reset together with axonal voltage once axonal voltage reaches the reset threshold. So the spike shapes seen at the soma are relatively small. Only the beginning parts of spikes are kept. But it suffices to analyze the impact of AP initiation site on spike shapes. We can see that the kink at the beginning of a spike is slightly larger when $P_{N a}=80 \mu m$. The phase plot Fig $36 \mathrm{D}$ confirms the observation. The slope of phase plot is steeper when the separation of AP initiation is larger. Driven by stochastic stimuli, the somatic voltage derivative also rises more quickly for large $P_{N a}$ as shown in Fig $36 \mathrm{~F}$. It should be noticed in Fig 36A that the spike generation at the AP initiation is also sharper when the initiation site is farther away from the soma. The phase plot of Fig $36 \mathrm{C}$ shows that the axonal voltage derivative is much larger for $P_{N a}=80 \mu \mathrm{m}$ than that of $P_{N a}=20 \mu \mathrm{m}$. The axonal voltage derivative difference is not a consequence of the stimulus strength. In Fig $36 \mathrm{E}$, the phase plots of the axonal voltage are generated with stochastic stimuli. The phase plot curves vary with the instantaneous stimulus that triggers the spike. But the variation is limited. The axonal voltage derivative difference is mainly 
resulted from the lateral current entering the soma. When the AP initiation site is close to the soma, as a current sink, there will be more current leaked into the soma during the generation of a spike. This leads to similar spike shapes seen from soma and seen from axon. When the AP initiation site is far away from soma, the lateral current leak is less, which results in a sharper spike at the AP initiation site.
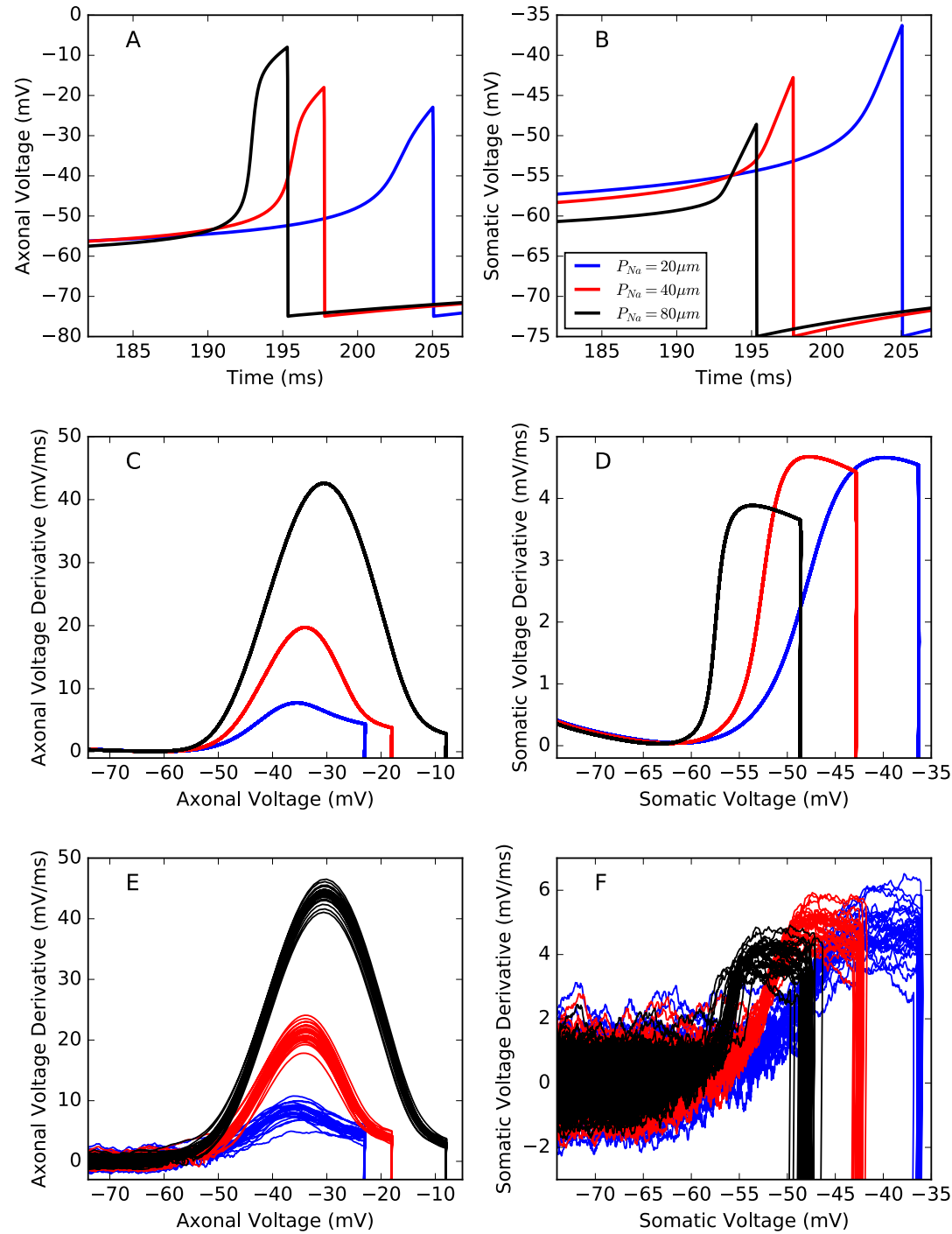

Fig 36. Sharper spike onset dynamics seen at soma for larger separations of AP initiation site. A, B: The spike shapes under constant inputs triggering a firing rate around $5 \mathrm{~Hz}$ in three conditions separately. C, D: The phase plots of the spiking voltages under constant inputs. Drastic drop of voltage derivative at the end of each curve corresponds to the negative voltage derivative at the voltage reset. E, F: The phase plots of spiking voltages under stochastic inputs triggering a firing rate of about $5 \mathrm{~Hz}$. The $\mathrm{CV}$ of inter spikes intervals is $0.85 \pm 0.05$. Correlation time of input is $5 \mathrm{~ms}$. All the figures share the same legend in B. 
For the next step, I examined the population encoding ability of the neuron model. If the axonal hypothesis can explain ultrafast population encoding, we should be able to reproduce high bandwidth encoding and sensitivity of dynamic gain to the correlation time of inputs $9,33,36$. Fig 37 shows the linear response functions of the multi-compartment model with different position of sodium channels. Simulation setup and denotations follow the methods section. In Fig 37A, I fixed the correlation time of inputs to $5 \mathrm{~ms}$. When the AP initiation site is $20 \mu \mathrm{m}$ from soma, the linear response function has a cutoff frequency of $10 \mathrm{~Hz}$, which is on the same scale of firing rates. In the intermediate frequency regime from $10 \mathrm{~Hz}$ to $100 \mathrm{~Hz}$, the linear response function decays with a slope close to -1 in log-log scale. So for a short correlation time of the input, linear response properties of the neuron model is similar to that of the conductance-based model 41. Increasing the separation distance of AP initiation site slightly enhances the dynamic gain in intermediate frequency regime. After that the dynamic gain decays faster than the $P_{N a}=20 \mu m$ case. In Fig $37 \mathrm{~B}$, I tuned the correlation time of input to $50 \mathrm{~ms}$. For a large correlation time, the cutoff frequencies of the linear response functions are still on the scale of 10 to $20 \mathrm{~Hz}$. The impact of AP initiation site is still limited. Fig $37 \mathrm{C}, \mathrm{D}$, and E compare the sensitivity of dynamic gain to the correlation time of inputs in three conditions. Having a larger correlation time only slightly enhance the resonance effect near the firing rate frequency. The dynamic gain after that decays faster than that of the short correlation time case.

In Fig 37, a larger correlation time makes the linear response functions more variable in high frequency regime. Related null hypothesis curves also have larger values for higher frequencies. This is because a larger $\tau$ makes the stimulus vary slower with time. To realize the same firing rate and CV of ISI, the mean of stimulus is larger, and the std of stimulus is smaller. This indicates the spikes are likely to be clustered in the time intervals when the stimulus slowly passes through the threshold to trigger spikes. This leads to the STA calculated from these spikes represent similar information of the stimulus. With the same firing rates, the final STA of a larger $\tau$ is more noisy. As a result, the linear response curves in higher frequency is less reliable. Each realization of the null hypothesis curve is more fluctuated in the high frequency part, so the final 95 percent upper bound is higher than that of the small correlation time.

From Fig 37 we can see that the axonal hypothesis can realize a more rapid AP initiation seen from the soma, but it is not sufficient to reproduce ultrafast population encoding. For a fixed correlation time of input, when $P_{N a}$ is equal to $80 \mu m$, the dynamic gain in the high frequency decays faster than those in the other two cases, which indicates that further separation of the AP initiation site will not enhance the encoding ability in high frequency regime. This implies that the conclusion of limited contribution of AP initiation site on dynamic gain is not an artifact of insufficient large parameter. Similarly, a larger correlation time of input could only lead to a further decay of dynamic gain in the high frequency regime.

Based on the results above, it indicates that the slow sodium activation function at the axon might be the fundamental reason for low cutoff frequency in linear response functions. The rapid onset dynamics probably should be a property indeed exists rather than a phenomenon seen from the soma. Separating AP initiation site from soma only makes the onset dynamics at higher voltage more rapid. The initial part of sodium activation function is still relatively slow. So it might be necessary to accelerate the 


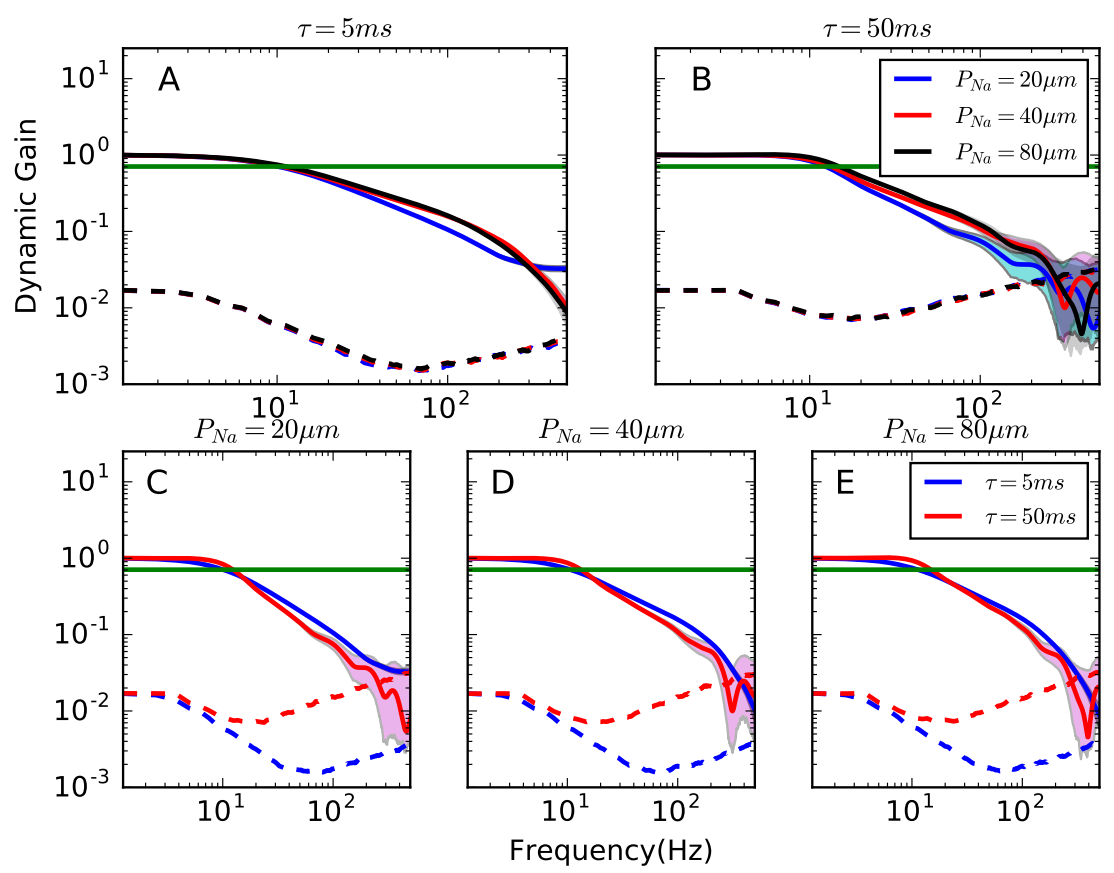

Fig 37. Linear response of the neuron model with a standard sodium activation curve. A, B: For a fixed correlation time of input, increasing the separation of AP initiation site from the soma has a limited impact on population encoding. Correlation time is denoted as $\tau$. The green horizontal line indicates at which frequency the dynamic gain decays by a factor of $\sqrt{2}$. The shaded areas around each curve are 95 percent confidence intervals. The dash line below is the 95 percent significance border for the null hypothesis that dynamic gain is zero. In A, the confidence intervals are thinner than the line widths. A and B share the same legend panel in B. C, D, E: For a fixed AP initiation site, increasing the correlation time of input does not enhance the dynamic gain in high frequency regime. C, D, and E share the same legend panel in E. The mean fire rate for all cases is $5 \mathrm{~Hz}$. The $\mathrm{CV}$ of inter spike intervals is $0.85 \pm 0.05$.

dynamics in this part as well. One way to realize this feature is to make the sodium activation dynamics more voltage sensitive, so that the sodium channels are activated avalanche-like once a threshold voltage is reached.

\subsection{The Impact of High Voltage Sensitivity of Sodium Channels on Population Encoding}

In this section, I will look into the impact of voltage sensitivity of sodium channels on the dynamic gain. I assume that the sodium channels are highly sensitive to the axonal voltage. When a spike is initiated, sodium channels are more likely to be activated avalanche-like together. To describe this assumption, I changed the parameter $k_{a}$ in sodium channel activation function from $6 \mathrm{mV}$ to $0.1 \mathrm{mV}$. Fig $38 \mathrm{~A}$ shows the activation function with the new $k_{a}$. Similarly as Fig $35 \mathrm{~B}$, I compared the sodium channel activation function with those representing it as the functions of somatic voltages. Different from the previous case, increasing $P_{N a}$ delays the activation. When the sodium activation function is sensitive to the axonal voltage, the 
neuron model can be regarded as passive before sodium activation. In this condition, injecting a constant input in the soma will lead to a voltage decrease from the soma to the AP initiation site. A larger $P_{N a}$ leads to a larger voltage difference. When reaching the same sodium activation threshold at the AP initiation site, the somatic voltage should be larger when $P_{N a}$ is larger. It is equivalent to a delay of the sodium activation function seen from the soma.
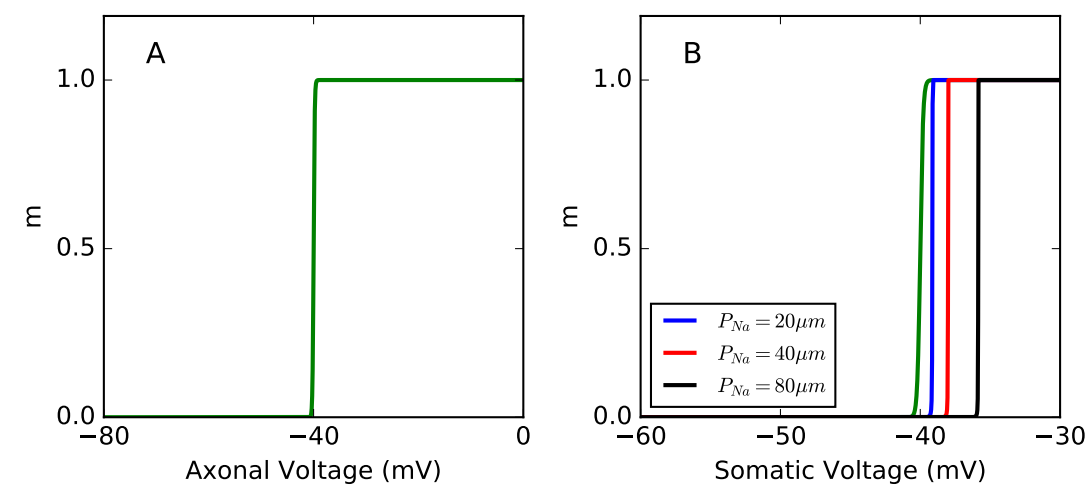

Fig 38. Sodium activation curve with a high intrinsic voltage sensitivity graphed versus axonal and somatic voltage. A: The sodium activation curve is represented as a function of axonal voltage. Activation parameter is $k_{a}=0.1 \mathrm{mV}$. B: Represented as the function of somatic voltage, the sodium activation variable has higher activation voltages when the AP initiation site is further away from the soma. The green curve is the activation curve from A for comparison.

With respect to spike shapes and phase plots seen from the axon, Fig 39 A, C, and E show that they share a similar feature to those in Fig 36. Separating AP initiation site from the soma reduces the current leaked into soma when generating a spike. So the spike shape and the voltage derivative are larger when $P_{N a}$ is larger. But seen from the soma, a larger $P_{N a}$ delays the rise of the voltage derivative in the phase plot Fig $39 \mathrm{D}$. This is in accordance with the observation of the sodium activation functions. In Fig $[39 \mathrm{~F}$, injecting the stochastic stimulus into the soma, the neuron model with a smaller $P_{N a}$ has a sharper AP initiation dynamics. The slope in the phase plot is steeper and less affected by the stochastic fluctuations when emitting spikes. The sodium activation at the axon is already well approximated by a step-like function. Increasing the separation distance of the AP initiation site has very limited effect on the activation function seen at the soma. Here the major difference is the amplitude of lateral current entering the soma for generating spikes. A larger $P_{N a}$ leads to a smaller lateral current, which results in a less sharper spike in the soma.

Fig 40A, B provide the linear response functions for different $P_{N a}$ values under the same $\tau$. The bootstrapping confidence intervals are thinner than the line widths in Fig 40A. Compared with the linear response functions with a standard sodium activation function, the dynamic gain in the high frequency regime is enhanced when high voltage sensitivity of sodium activation dynamics is introduced. The linear response functions become more flat in the high frequency part, which indicates that the neuron model can be well approximated as a LIF neuron when the AP initiation site is close to the soma. Separation of the AP initiation site acts as a low pass 

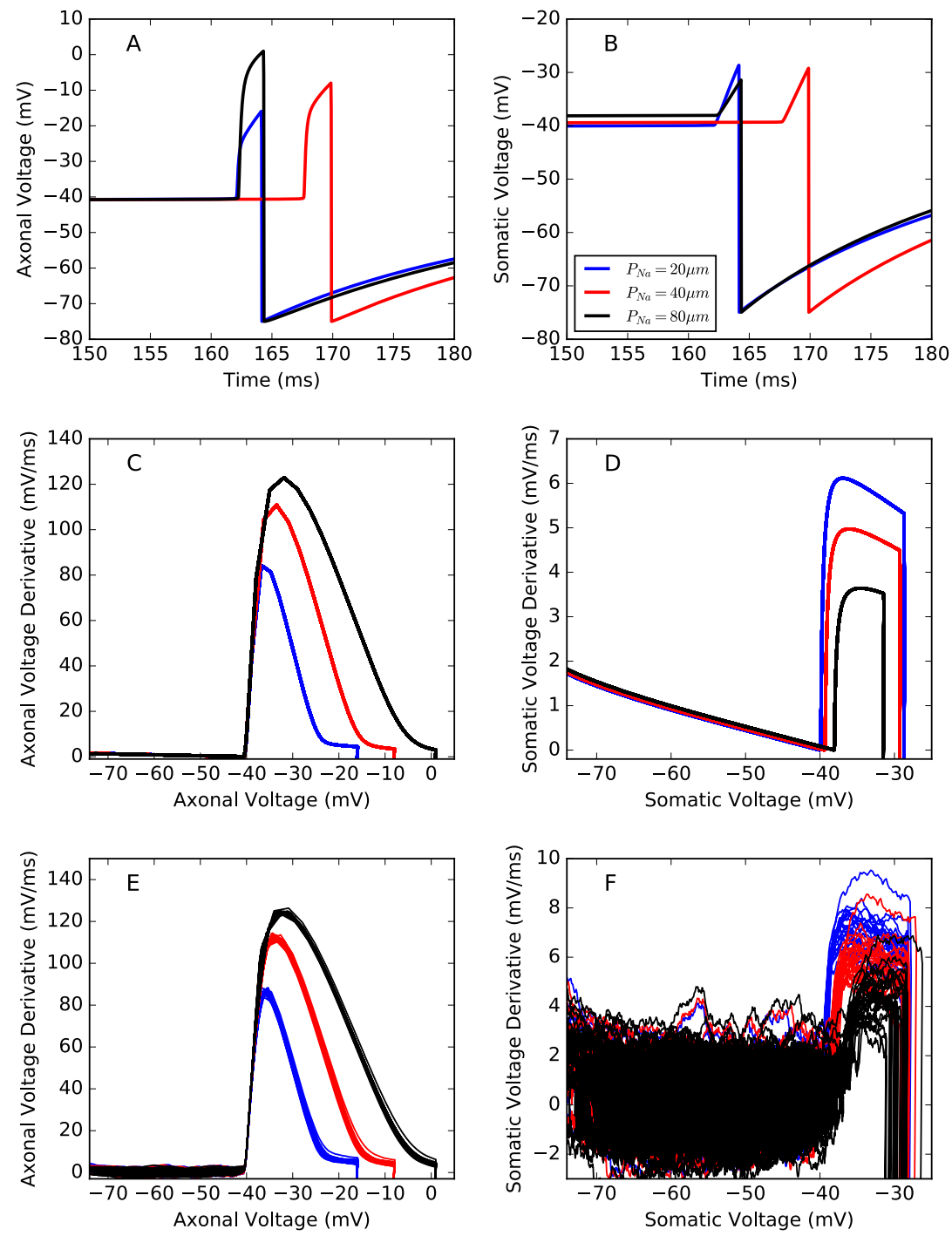

Fig 39. Sharper spike onset dynamics at the soma for smaller separations of AP initiation site. A, B: The spike shapes under constant inputs triggering similar firing rates in three conditions separately. C, D: The phase plots of the spiking voltages under the constant inputs. Drastic drop of voltage derivative at the end of each curve corresponds to the negative voltage derivative at voltage reset. E, F: The phase plots of the spiking voltages under the stochastic inputs triggering a firing rate of about $5 \mathrm{~Hz}$. The $\mathrm{CV}$ of inter spikes intervals is $0.85 \pm 0.05$. Correlation time of the input is $5 \mathrm{~ms}$. All the figures share the same legend in B.

filter on the dynamic gain. The neuron model has worse encoding at high frequencies. Tuning the correlation time of input to $50 \mathrm{~ms}$ in Fig $40 \mathrm{~B}$, the linear response functions reproduce the high cutoff frequencies observed in experiments 9 33 36. The AP initiation plays the same role as that in Fig 40A. In Fig 40 C, D, and E, we show the impact of correlation time on the dynamic gain for different $P_{N a}$.

The simulation results above show that when high voltage sensitivity 


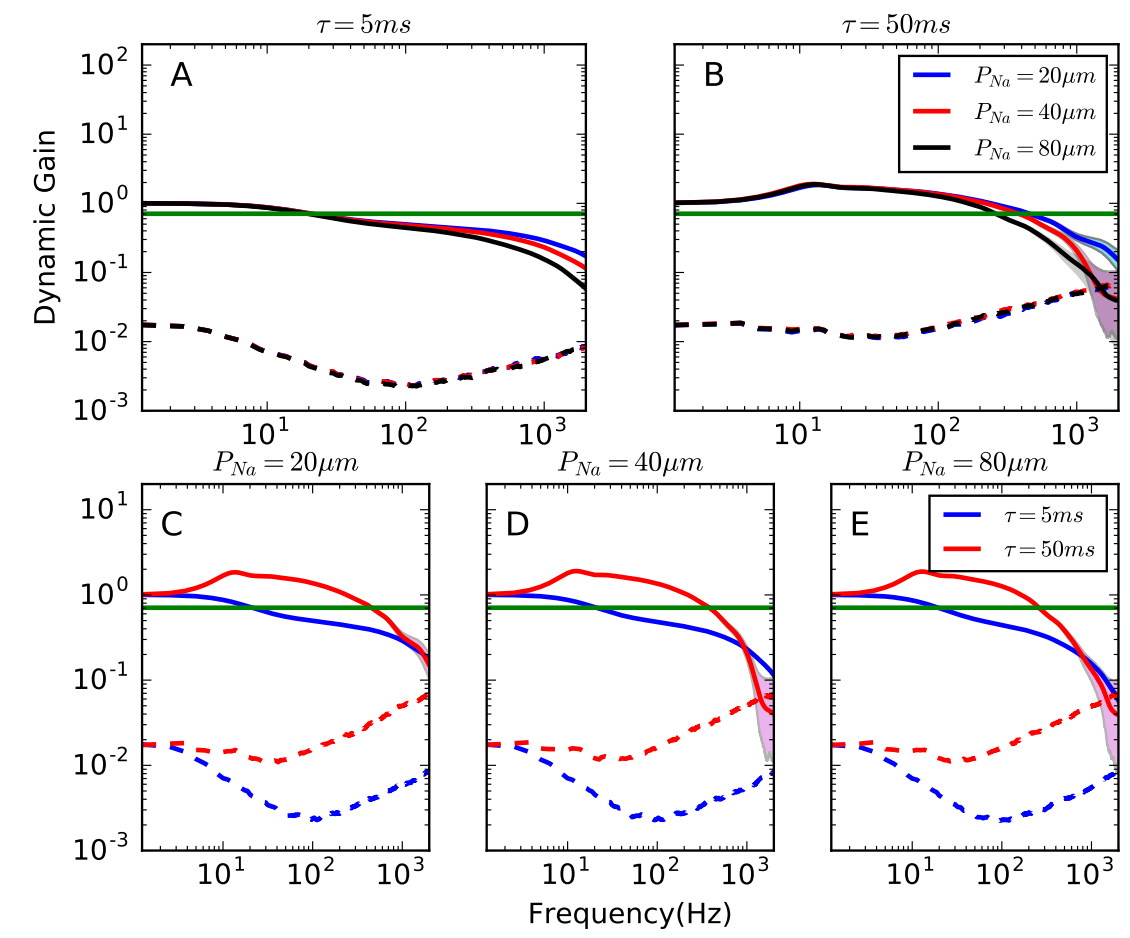

$\tau=50 \mathrm{~ms}$

Fig 40. Population dynamic gain with the high voltage

sensitivity sodium activation curve. A, B: For a fixed input

correlation time, increasing the separation of the initiation site reduces the dynamic gain in high frequency regime. The denotations in the figure are the same as those of Fig 37. In A, the confidence intervals are thinner than the line widths. A and B share the same legend panel in B. C, D, E: For a fixed initiation site, increasing the correlation time of inputs enhances the dynamic gain in the high frequency regime. C, D, and E share the same legend panel in E. The mean fire rate for all cases is $5 \mathrm{~Hz}$. The $\mathrm{CV}$ of inter spike intervals is $0.85 \pm 0.05$.

is introduced into the sodium activation dynamics, both high bandwidth encoding and enhanced dynamic gain with large correlation time are reproduced. A larger separation distance of the AP initiation site can not further accelerate the sodium activation dynamics seen from the soma. The major impact it has on population encoding is reducing the dynamic gain at high frequencies.

\subsection{Somatic and Axonal Voltage Dissociation}

Up till now, I have shown that with a standard sodium activation function, the axonal hypothesis can reproduce sharp AP onset seen at the soma. But it is not sufficient to reproduce fast population encoding. Fast population encoding is not equivalent to sharp spikes seen at the soma. Adding high voltage sensitivity to the sodium channels can fix the encoding ability. It remains unclear what properties of the standard sodium activation leads to the low cutoff frequencies in linear response functions. In this section, I will provide an explanation for the discrepancies between sharp spikes and fast population encoding. 
The spikes seen at the soma are shaped by the lateral current entering the soma, which is a reflection of the sodium current in the axon. The sharp initiation of a spike is a result of the instantaneous change of the lateral current. Based on the view of axonal hypothesis, we can regard it as the sodium current generated at the soma. The somatic sodium activation function is the axonal sodium activation function represented as the somatic voltage, as shown in Fig $35 \mathrm{~B}$. The step-like change in the sodium activation function seen from the soma describes the dissociation of axonal voltage and somatic voltage during a spike. When a spike is generated in the axon, the axonal voltage at the AP initiation site is brought up by the sodium current. The somatic voltage is still at a lower level due to the resistance in between. So we can see a high sodium activation ratio at a low somatic voltage. A steeper sodium activation function in Fig $35 \mathrm{~B}$ indicates a faster dissociation of the somatic voltage and the axonal voltage.

In Fig 41, I plot the dissociation dynamics between these two voltages under different AP initiation sites. The plot is generated by constant inputs. Here the reset voltage is removed so that we can see the trend of trajectories in the limit of large voltages. Continuous lines represent the dissociation dynamics of the neuron model with a standard sodium activation function. The green diagonal line is for reference. For different positions of sodium channels, all three curves stay close or below the green diagonal line. When the axonal voltage is low, almost all the sodium channels are closed. The somatic voltage is slightly larger than the axonal voltage if we inject a constant input in the soma. But here in the figure it is negligible. When the axonal voltage gets larger, more sodium channels are activated. The axonal voltage becomes larger than the somatic voltage. Two voltages start to dissociate. At the early stage, the dissociation rate is slow. This matches to the slowly rising part of the sodium activation functions in Fig $35 \mathrm{~B}$. A larger separation of the AP initiation site leads to a faster voltage dissociation in higher voltage regime. When the sodium channels are all activated, the sodium current amplitude is determined by the voltage difference between the axonal voltage and balanced voltage of sodium channels. So in this voltage regime, the dissociation is slowly getting smaller.

I also plot the voltage dissociation for the model with high voltage sensitivity in sodium activation function. The dash lines are used, and the color code is the same as the continuous ones. Different from the previous case, the dissociation curves are above the diagonal line until around $-40 \mathrm{mV}$, then they switch to the other side of the diagonal line. A larger $P_{N a}$ leads to a larger dissociation. In this case $P_{N a}$ has no significant effect on dissociation speed, all three dissociation dynamics are instantaneous.

Through comparison we can see that when $P_{N a}=80 \mu m$, the voltage dissociation dynamics are fast in the higher voltage regime for both types of sodium activation functions. The major difference of these two curves is the beginning part, which might be the cause of low cutoff frequency in the population encoding. A slow dissociation of the somatic and the axonal voltages corresponds to the slow rising part of the sodium activation function. The spike timing will be more perturbed by the stochastic stimulus if the spike initiation is slow. A sharp spike voltage rising after the slow spike initiation can be reproduced with the separation of AP initiation site, but it doesn't improve the encoding ability of the neuron model. With a high voltage sensitivity of the sodium channels, sodium currents are suppressed before the stimulus drives the voltage to spiking threshold. When a spike is initiated, sufficient amount of the sodium current are triggered in a short 


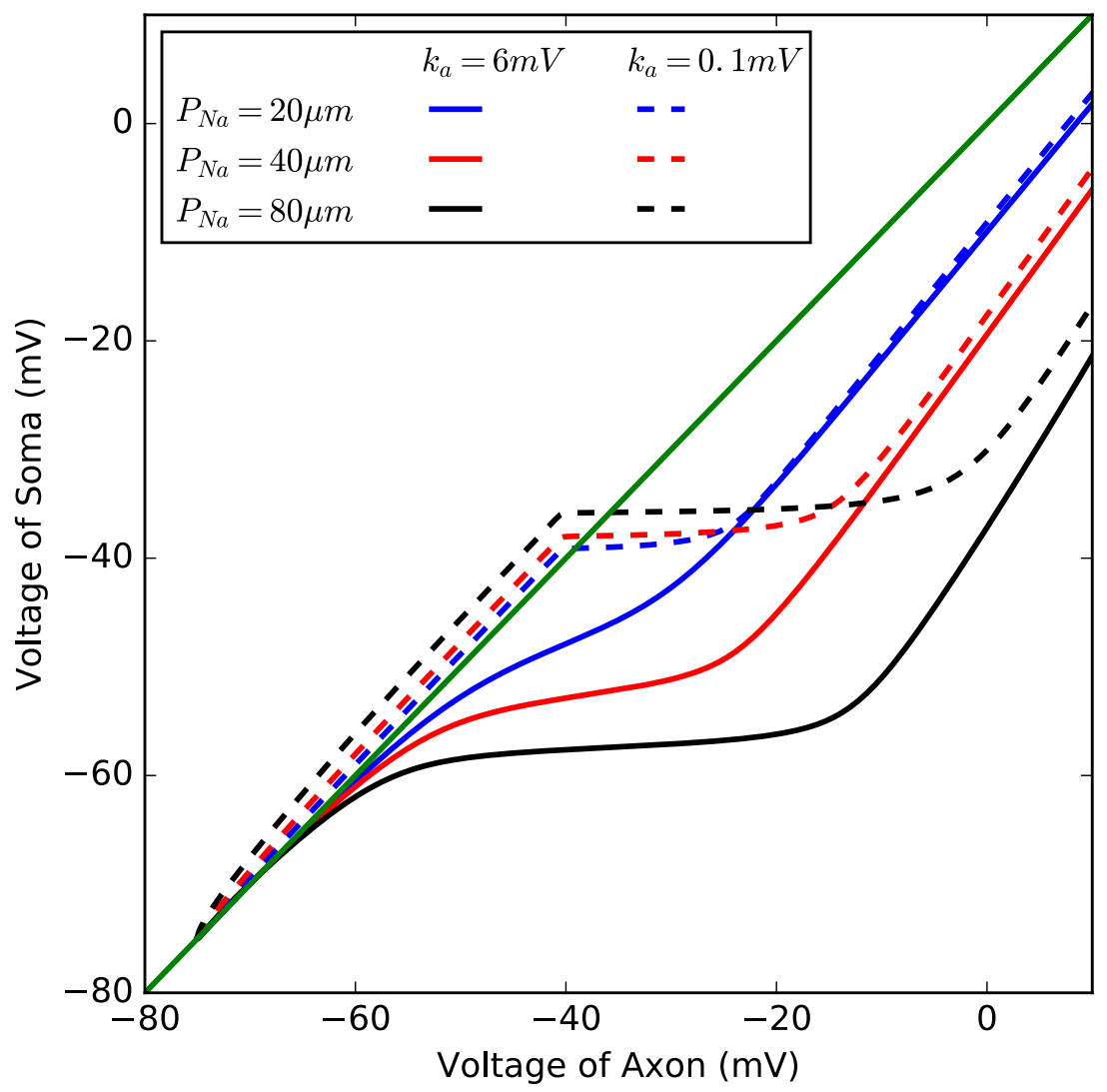

Fig 41. Dissociation of somatic and axonal voltages with and without high voltage sensitivity of the sodium channels. The continuous lines are the dissociation curves of the models with standard sodium activation dynamics. The dash lines are the dissociation curves of the models with high voltage sensitivity of the sodium activation dynamics. The green line is the diagonal line.

time to bring the voltage high enough for spike detection. In this sense, it avoids the random perturbation of the spike time. Reflected in the voltage dissociation dynamics, it is the close coupling of the somatic and axonal voltage before a spike is initiated and a rapid dissociation during spike generation.

\subsection{Simplified Model Representation}

In this section, I will further examine the contributions of different parameters to the dynamic gain for the neuron model having high voltage sensitivity in sodium channels. We will simplify the multi-compartment model into two equations describing the somatic voltage and the axonal voltage. With this simplification, we can obtain the linear response function analytically. From the approximations we can see that the position of sodium channels play a limited role in determining the cutoff frequency. Instead, the diameter of the soma has a large impact on the encoding.

First I will introduce the reduced model. The soma is represented as a sphere of diameter $d$. We assume it is isopotential of voltage $V_{s}$. The somatic membrane is passive with specific membrane leak conductance 
and capacitance denoted as $g_{L}$ and $c_{m}$. The soma receives an externally injected current and a lateral current from the axon. The AP initiation site is represented by a cylinder of diameter $a$ and length $a$. $a$ is much smaller than $d$. The voltage at this place is denoted as $V_{a}$. The specific membrane leak conductance and capacitance are denoted as $g_{L}$ and $c_{m}$. The action potential initiation site receives the lateral current from the soma and the sodium current. Only the activation part of the sodium channels will be included. Once $V_{a}$ reaches a threshold $\psi, V_{a}$ and $V_{s}$ will be reset to the resting potential $E_{p a s}$ simultaneously. The soma and the AP initiation site is connected by a cylinder shape resistance. The diameter of the cylinder is $a$. The length of the cylinder is denoted as $P_{N a}$. The intracellular resistance is denoted as $r_{L}$. The lateral current is determined by the voltage difference and the resistance in between. Below are the equations and a table of abbreviations.

$$
\begin{gathered}
\pi d^{2} c_{m} \frac{d V_{s}}{d t}=\pi d^{2} g_{L}\left(E_{\text {pas }}-V_{s}\right)+\frac{V_{a}-V_{s}}{\frac{r_{L} \cdot P_{N a}}{\frac{\pi a^{2}}{4}}}+I_{e} \\
\pi a^{2} c_{m} \frac{d V_{a}}{d t}=\pi a^{2} g_{L}\left(E_{\text {pas }}-V_{a}\right)+\frac{V_{s}-V_{a}}{\frac{r_{L} \cdot P_{N a}}{\frac{\pi a^{2}}{4}}}+\pi a^{2} \bar{g}_{N a}\left(E_{N a}-V_{a}\right) m\left(V_{a}\right)
\end{gathered}
$$

\begin{tabular}{|l|l|}
\hline$d$ & Diameter of soma \\
\hline$c_{m}$ & Specific membrane capacitance \\
\hline$V_{s}$ & Somatic voltage \\
\hline$t$ & Time \\
\hline$g_{L}$ & Specific leak conductance \\
\hline$E_{\text {pas }}$ & Resting potential \\
\hline$V_{a}$ & Axonal voltage \\
\hline$r_{L}$ & Intracellular resistance \\
\hline$P_{N a}$ & Distance between soma and AP initiation site \\
\hline$a$ & Diameter and length of AP initiation site \\
\hline$I_{e}$ & Electrode current \\
\hline $\bar{g}_{N a}$ & Specific sodium conductance \\
\hline$E_{N a}$ & Sodium balanced potential \\
\hline$m$ & Sodium channel activation function \\
\hline$\psi$ & Spike threshold \\
\hline
\end{tabular}

To calculate the linear response curve, the electrode current is represented as Eqn (20). For simplicity, we will represent the sodium activation function as a Heaviside function, such that below $\psi=-40 \mathrm{mV}$ it is 0 , above $-40 m V$, it is 1 . Once $V_{a}$ reaches $-40 m V$, we will reset $V_{s}$ and $V_{a}$ to $E_{\text {pas }}$. In this way, we can omit the sodium current term. Denoting $E_{p a s}-V_{a}$ as $V, V_{a}-V_{s}$ as $w$, we can simplify the equations as below.

$$
\begin{gathered}
c_{m} \frac{d V}{d t}=-g_{L} V+\frac{w}{4 r_{L} \cdot P_{N a}} \\
c_{m} \frac{d w}{d t}=-\left(g_{L}+\frac{a^{2}+d^{2}}{4 r_{L} \cdot P_{N a} \cdot d^{2}}\right) w-\frac{I_{e}}{\pi d^{2}}
\end{gathered}
$$

Denote $\frac{1}{4 g_{L} \cdot r_{L} \cdot P_{N a}}$ as $C_{P_{N a}}$. Denote $\frac{a^{2}+d^{2}}{d^{2}}$ as $r_{d}$. Denote $\frac{c_{m}}{g_{L}}$ as $\tau_{V}$. Denote $\frac{c_{m}}{g_{L}\left(1+\frac{a^{2}+d^{2}}{4 g_{L} \cdot r_{L} \cdot P_{N a^{\prime}} d^{2}}\right)}$ as $\tau_{w}$. The equations can be further simplified to: 


$$
\begin{gathered}
\tau_{V} \frac{d V}{d t}=-V+C_{P_{N a}} \cdot w \\
\tau_{w} \frac{d w}{d t}=-w-\frac{I_{e}}{\pi d^{2} \cdot g_{L}\left(1+r_{d} \cdot C_{P_{N a}}\right)}
\end{gathered}
$$

According to the parameters we chose for simulation, $\frac{1}{g_{L}}$ is $R_{m}$, which is $30000 \Omega \cdot \mathrm{cm}^{2} \cdot r_{L}$ is $150 \Omega \cdot \mathrm{cm} . P_{N a}$ is chosen to be $20 \mu \mathrm{m} . \tau_{V}$ is $22.5 \mathrm{~ms}$. $C_{P_{N a}}$ is 25000 , far larger than $1 . d$ is much larger than $a$, so $r_{d}$ can be approximated as 1 . These approximations imply that $\tau_{w}$ is far smaller than $\tau_{V}$. We can thus substitute $w=-\frac{I_{e}}{\pi d^{2} \cdot g_{L}\left(1+r_{d} \cdot C_{P_{N a}}\right)}$ directly into Eqn 36 . $\frac{C_{P_{N a}}}{1+r_{d} \cdot C_{P_{N a}}}$ can be approximated by 1 . Then our final equation becomes:

$$
\tau_{V} \frac{d V}{d t}=-V-\frac{I_{e}}{\pi d^{2} \cdot g_{L}}
$$

Taking the Gauss-Rice approach [4, 47], the linear response function can be represented as:

$$
r(\omega)=\left(\frac{\theta}{\sigma_{V}^{2}}+i \omega \sqrt{\frac{\pi}{2}} \frac{1}{\sigma_{\dot{V}}}\right) \frac{1}{1+i \omega \tau_{V}}
$$

Here $\theta$ is the voltage distance between the average voltage under the stochastic input and the spike threshold $\psi . \sigma_{V}$ is the standard deviation of $V$, which is also the standard deviation of $V_{a} . \sigma_{\dot{V}}$ is the standard deviation of $\dot{V}$, which is also the standard deviation of $\dot{V}_{a}$. For this simple model, we can obtain $\sigma_{V}$ and $\sigma_{\dot{V}}$ analytically where $\sigma_{V}^{2}=\frac{\sigma^{2} \tau}{\left(\pi d^{2} g_{L}\right)^{2}\left(\tau+\tau_{V}\right)}$, $\sigma_{\dot{V}}^{2}=\frac{\sigma^{2}}{\left(\pi d^{2} g_{L}\right)^{2}\left(\tau+\tau_{V}\right) \tau_{V}}$. Substituting these two terms into Eqn 39 and taking the absolute value, we obtain the dynamic gain. With a difference of a coefficient, we can represent the formula as:

$$
|r(\omega)| \propto \sqrt{1+\omega^{2} \frac{\tau_{V} \tau^{2} \sigma^{2}}{2 \pi\left(\tau_{V}+\tau\right) \theta^{2} g_{L}^{2} d^{4}}} / \sqrt{1+\omega^{2} \tau_{V}^{2}}
$$

Based on the formula we can see that the linear response function is most sensitive to the soma diameter $d$. Decreasing $d$ can increase dynamic gain which improves the ability of population encoding. This observation is valid when the soma diameter is large enough such that the multi-compartment model can be well approximated as an integrate and fire model. When the soma size is small, the role of lateral current will become more important. Comparing the analytical formula here and the simulation results with $k_{a}=0.1 \mathrm{mV}$, one major difference is the dynamic gain in the high frequency limit. Here the dynamic gain doesn't decay in the high frequency. This difference is originated in the filtering of the high frequency components of the stimulus when transmitting from soma to AP initiation site. At the AP initiation site, the spikes are triggered by the filtered stimulus. However, when calculating the linear response function, the Fourier transform of the STA is divided by the unfiltered stimulus injected in the soma. That's why we can see a decay of the dynamic gain in the simulation results.

\subsection{Discussion}

Linear response analysis of neuron population encoding is first proposed by Knight [37. Recently it is introduced in experiments [9, 33 $34,36,91$. It is 
found that cortical neurons are capable of ultrafast population encoding. The cutoff frequencies of the linear response function are above $100 \mathrm{~Hz}$ which are much higher than the firing rates of individual neurons. The dynamic gain in the high frequency regime is enhanced when increasing the correlation time of the stimulus 36. Rapid AP onset is proposed to be closely associated with these properties 7]. Reducing AP onset rapidness impairs the encoding ability [9. Several hypotheses have been proposed to explain the ultrafast population encoding and rapid AP onset [7, 48, 49]. One hypothesis proposed that rapid AP onset seen at the soma is caused by the separation of AP initiation site from the soma 48. A larger separation of the AP initiation site can make the spikes sharper seen from the soma. In this way, it can realize ultrafast population encoding. In this chapter, I calculated the linear response function of the neuron model and examined the impact of AP initiation site on population encoding. With a standard sodium activation function, the neuron model has a low cutoff frequency on the scale of the firing rate. A larger separation of the AP initiation site has a limited impact on the encoding ability. Increasing the correlation time of input does not enhance the dynamic gain in high frequency regime. This indicates that sharp spikes in the soma is not equivalent to ultrafast population encoding. Adding voltage sensitivity to sodium activation dynamics can recover the features of ultrafast population encoding. Comparing somatic and axonal voltages dissociation dynamics, I proposed that the slow sodium activation dynamics at the initiation of a spike is more likely to be affected by the stochastic stimulus. This undermines the spike time detection precision. Sharp spikes rising from the fluctuating voltages correspond to the large sodium current after the spike initiation, which doesn't reduce the impact of stochastic stimulus on spike time detection. High voltage sensitivity of the sodium activation dynamics reduces the spike initiation time which can further reduce the uncertainty in spike time detection. Based on our results, AP generation dynamics are the key factors for realizing ultrafast population encoding. Without a proper spike generation mechanism, separation of the AP initiation site alone is not sufficient to explain ultrafast population encoding.

Comparing the phase plots of spike generation dynamics in Fig 36 and Fig 39, we can see that the contributions of AP initiation site to spike generation are mainly in two aspects. One is the size of the lateral current. When the AP initiation site is closer to the soma, there will be a larger lateral current entering the soma when a spike is generated. The voltage derivative of the spike is smaller. In comparison, when the AP initiation site is far from the soma, the AP generation is more isolated from the soma. There will be less lateral current which leads to larger voltage derivative during a spike. A lager separation of the AP initiation site slightly enhanced the dynamic gain in intermediate frequency regime. When the sodium activation function is like a step function, the impact of lateral current is limited. A large amount of the sodium current is triggered when a voltage threshold is reached. The AP onset dynamics are much more rapid in the axon as seen in Fig 39. The size of the lateral current change the maximum voltage derivative during a spike. But AP onset dynamics are almost unaffected. In this case, the major impact of the AP initiation site is the filtering of the stimulus. The stochastic stimulus is injected in the soma and transmitted into the AP initiation. During the transmission, the high frequency components of the stimulus is reduced more than the low frequency components. A larger separation of the AP initiation site 
has a larger filtering of the high frequency components. The spike times generated by the filtered stimulus have less contribution from the high frequency components. When calculating the linear response function, the Fourier transform of the STA is divided by the power spectral density of the stimulus injected in the soma. So the linear response functions show a worse encoding ability for the high frequency regime when the AP initiation site is farther away from the soma as shown in Fig 40. Here in our current model, the soma is simplified as a passive compartment. For more general case, if the ion channels are added to the soma, injecting a stimulus into the soma may trigger additional ion current entering the AP initiation site. The linear response function may have a higher bandwidth than that generated by injecting the stimulus directly into the AP initiation site.

Besides the axonal hypothesis, another hypothesis proposed cooperative gating of sodium channels to explain the rapid AP onset and ultrafast population encoding [43, 46]. According to the hypothesis, it proposes that when generating spikes, the activation of one sodium channel may increase the activation probability of neighboring sodium channels. In this sense, the activation function of individual sodium channel might be in the form of the standard sodium activation functions in our multi-compartment model, but for a collection of sodium channels, the total sodium activation current will display instantaneous activation. Following the cooperative gating hypothesis, when the voltage is low, the total sodium activation current is well approximated by the sodium current generated by a group of independent sodium channels. This implies that if the sodium activation function of the individual channel has slow initiation dynamics, the collective sodium activation function should have a slow initiation part followed by an instantaneous rising phase. Based on the results in our work, a slow initiation part may undermine the population encoding ability. So it requires that the cooperative gating strength should be strong enough such that the sodium current rise rapidly at a low voltage. The slow AP initiation regime is removed. The sodium activation current should be small enough before a threshold across which sufficient amount of sodium current is triggered for spike generation. In this way, fast AP initiation reduces the perturbation of the stochastic input to the spike time detection. High voltage sensitivity is similar but not equivalent to the cooperative gating hypothesis.

One recent study [49] highlighted the importance of dendrite size in determining cutoff frequency of the linear response function. One interesting observation is that without the dendritic tree, the neuron model can realize a high cutoff frequency over $100 \mathrm{~Hz}$ in its linear response function. The multi-compartment model has a complete AP generation mechanism which includes sodium activation, sodium inactivation and potassium current. Cooperative gating and high voltage sensitivity are not required in the AP generation dynamics. It seems to indicate that ultrafast population encoding can be realized without any assumptions on the AP generation dynamics. However, it doesn't examine whether the dynamic gain in the high frequency is sensitive to the correlation time of the stimulus. It is worth exploring the underlying mechanisms of high cutoff frequencies in its linear response functions. 


\section{The Implications of High Voltage Sensitiv- ity}

In this chapter, I will present a few implications derived from high voltage sensitivity of the sodium activation function. In the previous chapter, I have shown that with a standard sodium activation function, the multicompartment model proposed by Brette fails to reproduce fast population encoding. However, based on the axonal hypothesis, the multi-compartment model in [48] is considered to be equivalent to the cooperative gating model, which claims to realize rapid AP onset and high bandwidth encoding 46. So in this chapter, I will first take a closer look at the cooperative gating model and examine whether it is able to realize fast population encoding. Second, since the slow sodium activation is the cause of low cutoff frequency in the linear response function, can we enhance the encoding ability by increasing the voltage sensitivity in the low voltage regime without introducing unrealistic high activation slope in higher voltage regime? Third, in the previous chapter, I failed to see a clear impact of AP initiation site on population encoding, so in what conditions can AP initiation make a significant contribution to linear response function? Fourth, I also have shown that seen from the equations, linear response function is most sensitive to the soma size. But this is based on the requirement that other parameters such as stimulus and neuron morphology are fixed. When calculating the linear response functions, we control the firing rate and firing patterns such that the neuron model's "working conditions" are fixed. Does the strong impact observed in the formula still apply under this evaluation criterion?

I will show that based on the parameters given in [46], which is $10 \%$ of strong cooperative gating sodium channels, it is not able to reproduce high bandwidth encoding. Also the dynamic gain is not sensitive to the correlation time of the background current. Increasing the ratio of cooperative gating sodium channels does not reproduce ultrafast population encoding, but make the model unable to generate spikes properly. Large stimulus fluctuations may make the voltage trapped in the intermediate voltage. One way to fix this problem is to have small std in the stimulus and small time steps in simulation. However, this solution does not recover high bandwidth encoding. Reducing the cooperativity makes it possible to have larger ratio of cooperative gating sodium channels. But this leads to slow AP initiation, which undermines the encoding.

Then I will show that with a piece-wise linear sodium activation function in Brette's model, the slow sodium activation at the beginning of spike generation is removed. Fast population encoding can be realized with a slope similar to that of the standard sodium activation function. With this sodium activation function, increasing the separation distance of AP initiation site can enhance the encoding ability.

In the last part of this chapter, I will examine the impact of soma size on the encoding ability of Brette's model. I will show that reducing the soma size can enhance the encoding ability when we fix firing rate and firing pattern.

\subsection{Population Encoding of the Cooperative Gating Model}

Previously I have shown that seen from the soma, the sodium activation dynamics become more rapid when the AP initiation site is moved away 
from the soma. If we take this representation as writing the axonal sodium activation dynamics into the somatic voltage equation, the resulting sodium activation dynamics shares similar features of the cooperative gating sodium channel dynamics as shown in Fig 15. Both activation curves keep the slow activation dynamics in the low voltage regime. When the voltage is large enough, the sodium activation variables rise rapidly. The rising speed depends on the separation distance of the AP initiation site or the cooperativity of the sodium channels. Based on these observations, the multi-compartment model in [48] is considered to be equivalent with the cooperative gating model.

Cooperative gating hypothesis proposes a biophysical mechanisms of sodium channels aimed to reproduce rapid AP onset and fast population encoding [46]. The hypothesis suggests that the activation of one sodium channel increases the activation probability of neighboring sodium channels, which is formulated as below:

$$
m_{\infty}^{J}=m_{\infty}\left(V+K J\left(m_{\infty}^{J}(V)\right)^{x} h\right)
$$

$m_{\infty}(V)$ is the gating variable of individual sodium channels. $m_{\infty}^{J}(V)$ is the gating variable of cooperative gating sodium channels. $K$ describes the number of neighboring sodium channels that are coupled together for cooperative gating. $J$ describes the coupling strength. $K J$ describes the cooperativity among sodium channels. $h$ is the inactivation variable of the individual sodium channels. $\left(m_{\infty}^{J}(V)\right)^{x} h$ is the open probability of cooperative gating sodium channels. In [46], $x$ is 3. At voltage $V$, the gating variable of cooperative gating sodium channels takes the value which is equivalent to that of the independent gating variable at $V+K J\left(m_{\infty}^{J}(V)\right)^{x} h$. A stronger cooperativity $K J$ implies a higher gating variable value at $V$. One observation is that when the voltage is very low, few sodium channels are activated. In this case, $\left(m_{\infty}^{J}(V)\right)^{x} h$ takes a small value so that the open probability of the cooperative gating channels are similar to that of the independent sodium channels. Fig 15 shows this feature.

In the implementation of cooperative gating models, it also changed the equation describing sodium gating variable. The factor of time constant of sodium channels $\tau_{f}$ is changed from 1 to 0.01 , which makes the sodium activation function tracks the stationary form better at corresponding voltages. Besides, the equation of sodium gating variable is in the form of $\tau_{m} \frac{d m^{3}}{d t}=m_{\infty}^{3}-m^{3}$, rather than $\tau_{m} \frac{d m}{d t}=m_{\infty}-m$. Since the time constant of the sodium channels are quite small, it is expected to make limited difference. In the following results, to analyze the encoding ability of the cooperative gating model, I will stick to the equation and parameters used in 46].

Fig 42 shows the comparison of linear response functions of the original Wang-Buzsaki model and the cooperative gating model. In this figure, the ratio of cooperative gating channels is $10 \%$ of the total sodium channels. The parameter $K J$ that describes the strength of cooperativity is set to 1000 . The stimulus of neuron model is composed of a constant input, a sinusoidal signal, and a zero-mean background noise generated with the OU process. The constant input is able to generate $10 \mathrm{~Hz}$ firing rate. The properties of stimulus are not specified in [46], so the CV of ISI is unknown. From the figure we can see that by adding cooperative gating sodium channels, the dynamic gain in low frequency and high frequency are enhanced. However, the cutoff frequencies of the linear response curves are still around 20 to 
$30 \mathrm{~Hz}$. Also, with more rapid AP onset, the dynamic gain in the high frequency is still insensitive to the correlation time of the stimulus. The encoding performance of the cooperative gating model does not fit with the experimental results 9,33 or the theoretical results of LIF neuron 39]. This seems to indicate that with the current parameters, it is not sufficient to reproduce high bandwidth encoding. To realize fast population encoding, it requires to increase the cooperativity and the ratio of cooperative gating sodium channels.

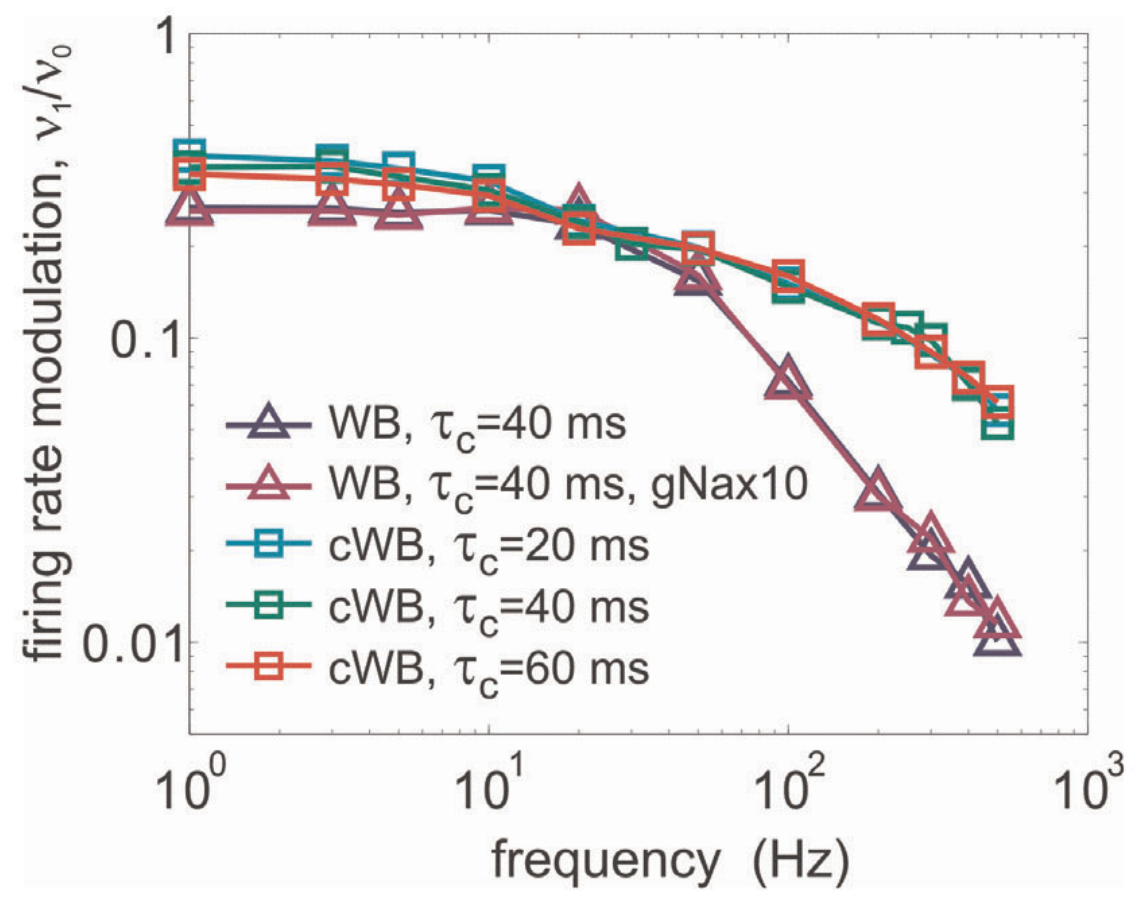

Fig 42. Linear response functions of cooperative gating models

from [46]. WB represents the original model without cooperative gating. cWB represents the cooperative gating model. KJ is set to be 1000 . The ratio of cooperative gating models is set to $10 \% . \tau_{c}$ is the correlation time of background current. According to the simulation results in [46, the original model fails to reproduce high bandwidth encoding. Increasing the sodium conductance doesn't change this feature. By introducing cooperative gating sodium channels, the dynamic gain in the high frequency regime is enhanced. But there is still no dependence on the correlation time of background current.

In the next section, I will examine the encoding ability of the cooperative gating model. I will first show that the model proposed in [46] and the example code provided in the supplementary material are two different models. The model in the paper has the cooperative gating of inactivation of sodium channels. While the model used in the code doesn't have the cooperative gating of inactivation of sodium channels. With the model provided in the code, it is not able to reproduce the linear response curves in Fig 42 when the stimulus is fluctuation-driven. Fluctuation-driven means the mean of stimulus is not able to trigger spikes. The spikes are triggered by the fluctuations in stimulus. If we increase the cooperativity or the ratio of cooperative gating sodium channels, the neuron model can't generate spikes properly. The major reason for this problem is the inactivation of 
sodium channels. If the sodium channels are deinactivated at a high voltage, large amount of sodium current will be available again which makes it impossible to bring down the voltage for the next spike. In this case, the voltage is blocked in some intermediate value. Adding cooperative gating to the inactivation variable $h$ can solve this problem. With cooperative gating of sodium inactivation, the sodium channels will be block till a low enough voltage before deinactivated, then the neuron model can generate spikes properly.

\subsection{Examination of the Cooperative Gating Model}

As the first step of examination of the cooperative gating model, I would like to first restate the definition of cooperative gating model with a more rigorous expressions and denotations.

According to the cooperative gating hypothesis, the stationary activation function is denoted as $m_{\infty}^{J}(V)$. Following the same denotation, the inactivation variable should be denoted as $h_{\infty}^{J}(V)$. Corresponding dynamic activation variable and inactivation variable should be denoted as $m^{J}(V)$ and $h^{J}(V)$. The time constants of these two variables should be $\tau_{m}^{J}$ and $\tau_{h}^{J}$. The definition of the cooperative gating model should be:

$$
\begin{gathered}
m_{\infty}^{J}(V)=m_{\infty}\left(V+K J\left(m_{\infty}^{J}(V)\right)^{x} h_{\infty}^{J}(V)\right) \\
\tau_{m}^{J} \frac{d m^{J}}{d t}=m_{\infty}^{J}-m^{J} \\
\tau_{m}^{J}=\tau_{m}\left(V+K J\left(m_{\infty}^{J}(V)\right)^{x} h_{\infty}^{J}(V)\right)
\end{gathered}
$$

As for the inactivation of cooperative gating sodium channels, if it doesn't have cooperative gating, then it has the same dynamics of standard inactivation variable. If it has the similar cooperative gating expression, then it should be:

$$
\begin{gathered}
h_{\infty}^{J}(V)=h_{\infty}\left(V+K_{h} J_{h}\left(m_{\infty}^{J}(V)\right)^{x} h_{\infty}^{J}(V)\right) \\
\tau_{h}^{J} \frac{d h^{J}}{d t}=h_{\infty}^{J}-h^{J} \\
\tau_{h}^{J}=\tau_{h}\left(V+K_{h} J_{h}\left(m_{\infty}^{J}(V)\right)^{x} h_{\infty}^{J}(V)\right)
\end{gathered}
$$

If the ratio of cooperative gating sodium channels is denoted as $p$, the equation for cooperative gating model should be:

$$
\begin{aligned}
c_{m} \frac{d V}{d t} & =g_{L}\left(V_{L}-V\right)+g_{N a}\left[p\left(m^{J}\right)^{x} h^{J}+(1-p)(m)^{x} h\right]\left(V_{N a}-V\right) \\
& +g_{K} n^{4}\left(V_{K}-V\right)+I_{e x t}
\end{aligned}
$$

Here $c_{m}$ is the specific membrane capacitance. $V$ is the membrane voltage of the single compartment neuron. $g_{L}$ is the specific leak conductance. $g_{N a}$ and $g_{K}$ are the specific conductance of sodium channels and potassium channels. $V_{L}, V_{N a}$, and $V_{K}$ are the balanced voltage of leak, sodium and potassium separately. $I_{\text {ext }}$ is the external input.

With the more rigorous denotations, I will point out the unclear parts in original model expressions. First, in the definition of cooperative gating sodium channels Eqn 41, it used the variable $h$ rather than $h^{J}$, which seems to imply that the inactivation of the cooperative gating model is the 
same of those without cooperative gating. However, in its equation for the cooperative gating model, the inactivation variable for cooperative gating sodium channels is $h^{J}$. Second, when showing the stationary cooperative gating sodium activation functions with different cooperativities, the inactivation variable of cooperative gating sodium channels is set to a constant denoted as $H_{0}$, which is 1 according to the author's thesis. Then in the expression of the cooperative gating model, it says that the inactivation curve of the cooperative channels $h_{\infty}^{J}$ has the same amount of voltage shift $h^{J}(V)=h\left(V+H_{0} K J m^{J}(V)\right)$, and $\tau_{h}^{J}(V)=\tau_{h}\left(V+H_{0} K J m^{J}(V)\right)$. The specific value of $H_{0}$ here is not given in the thesis. Third, in the code provided in the supplementary material, the inactivation of cooperative gating channels are the same with that of the standard sodium channels. It is not clear the linear response curves in Fig 42 are generated with which type of model. Also, for the model with the cooperative gating of sodium inactivation, it is not clear whether it is a constant parameter $H_{0}$, or it has the same form with that of activation function.

To examine the impact of cooperative gating of sodium channels, I first calculated the linear response functions based on the model provided in the code, which has no cooperative gating of sodium inactivation. Fig 43 shows the linear response curves of the original WB model and the cooperative gating model. The stimulus is tuned to reproduce $5 \mathrm{~Hz}$ firing rates. The CV of ISI is about 0.85 . The time step is set to $0.025 \mathrm{~ms}$. Without cooperative gating, the WB model has a low cutoff frequency in its linear response function. Increasing the correlation time of the stimulus only slightly enhances the dynamic gain in the low frequency regime. The linear response curve has a larger decay slope in the high frequency limit. With $10 \%$ of cooperative gating sodium channels, there is no significant differences in the linear response curves.
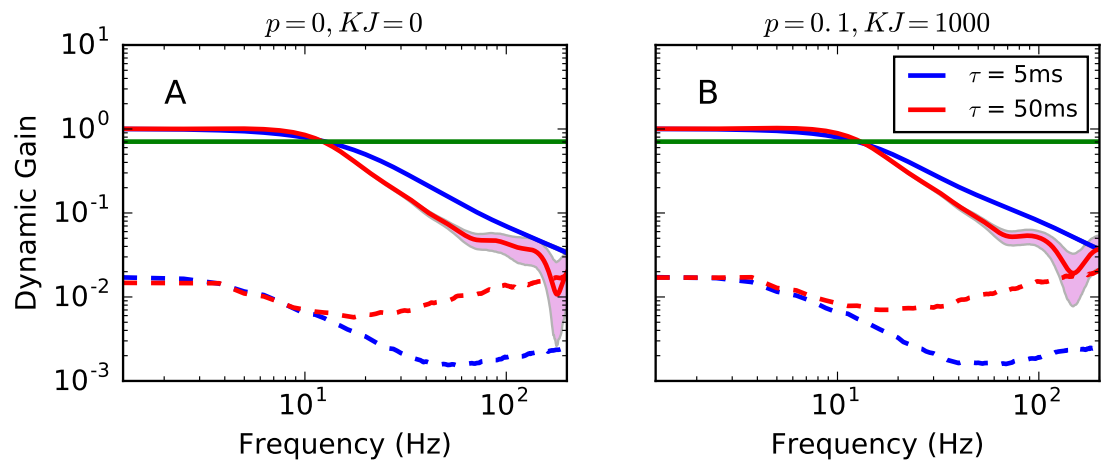

Fig 43. Simulation of the WB model without and with cooperative gating of the sodium channels. In both conditions, the neuron models fail to reproduce fast population encoding. With the given ratio and strength of cooperativity of the cooperative gating sodium channels, there is no significant improvement of the dynamic gain in the linear response curves. The firing rate is $5 \mathrm{~Hz}$. The CV of ISI is about 0.85 .

Note here are two differences in the simulation conditions compared to those in the original work. The first is the simulation method. In the original work, it injected the neuron model with a sinusoidal signal merged in the stochastic noise. The dynamic gain is measured as the tuning ratio between the amplitude of firing rate sinusoidal fluctuation and the amplitude of sinusoidal signal. In the figure above, I used the Fourier transform method. 
In the method chapter, I have shown that these two methods are equivalent with each other. So the difference in the dynamic gain is not a result of the simulation methods difference. The second difference is the properties of the stimulus. In the original method, the background noise is composed of a constant mean value, and a zero-mean stochastic stimulus generated with the OU process. The mean of the stimulus can generate $10 \mathrm{~Hz}$ firing rate. The std of the stochastic stimulus is not specified. It is not clear about the exact firing rate with the stochastic noise and sinusoidal signal. So it is possible that the neuron model firing is mean-driven. In my simulations above, the mean of the stimulus is not able to trigger spikes. The neuron model firing is fluctuation driven. The firing rate is $5 \mathrm{~Hz}$.

To examine the influence of stimulus properties on the encoding ability, I fixed the parameter $p$ and $K J$ to be 0.1 and 1000 , and find the constant input to trigger $10 \mathrm{~Hz}$ firing rates. Then I found the std of stimulus such that the CV of ISI is close to 0.8. With the simulation conditions above, I calculated the linear response function of models with and without the cooperative gating of the sodium inactivation. Fig 44 shows that in both cases, the linear response functions have no significant differences with those in Fig 43 .
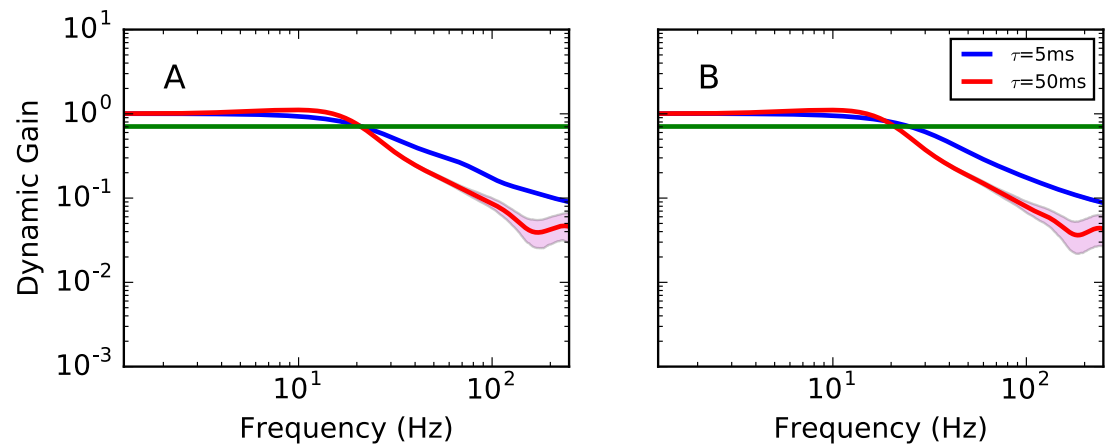

Fig 44. Linear response functions of the cooperative gating models without and with cooperative gating of sodium inactivation. The mean of stimulus can generate $10 \mathrm{~Hz}$ firing rate. The std of stimulus is tuned to make the CV of ISI about 0.8. In A, there is no cooperative gating of sodium inactivation. In $\mathrm{B}$, cooperative gating of sodium inactivation is added. For both models, no significant improvement of the encoding abilities are detected compared with the original WB model.

The limited difference in encoding between WB model and cooperative gating model with $p=0.1$ and $K J=1000$ can be explained by the uncertainty in spike time detection discussed in the method chapter. Fig 31 and 32 have shown that the uncertainty of spike time detection for the WB is mainly generated in the first $10 \mathrm{mV}$ after the spike generation bifurcation. Setting the spike detection voltage $10 \mathrm{mV}$ after the bifurcation voltage, the corresponding linear response functions are quite similar to those with spike detection voltage set at much larger value. Fig 45 shows the activation function of cooperative gating sodium channels used in [46. Injecting the stochastic stimulus that generated the linear response function, I recorded the voltage and sodium activation function for the rising phase of the first spike. The activation function rises slowly in low voltage regime. When 
reaching about $-50 \mathrm{mV}$, the activation function rises instantaneously to 1 . The bifurcation voltage for the original $\mathrm{WB}$ model is at about $-60 \mathrm{mV}$. So we can see that the rapid onset at $-50 \mathrm{mV}$ fails to erase the spike detection uncertainty. Even if all the sodium channels have the cooperative gating activation dynamics, the neuron model can't reproduce fast population encoding.

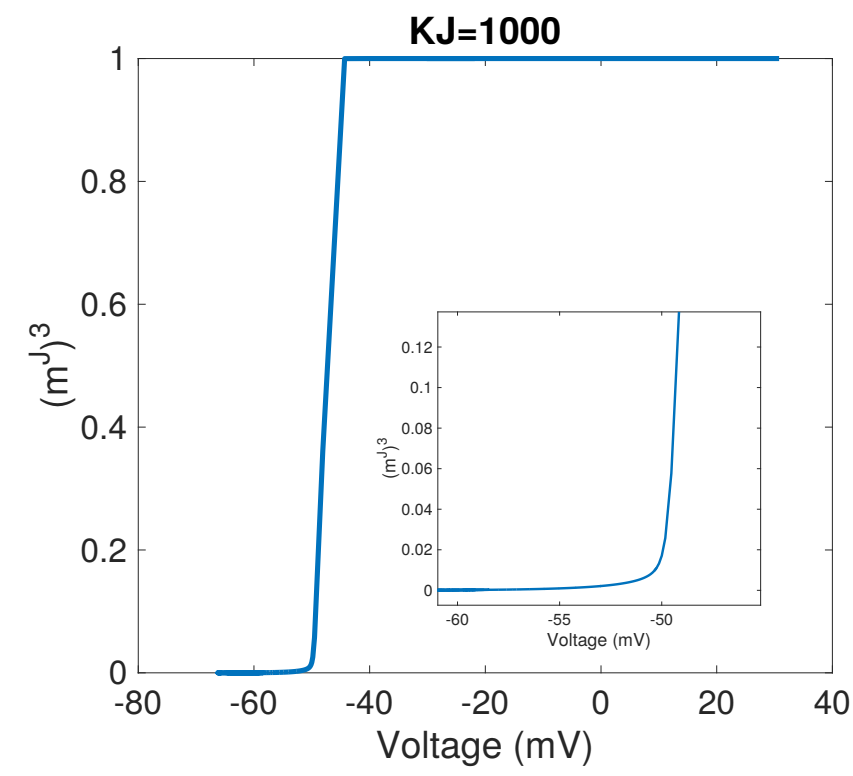

Fig 45. Activation function of the cooperative gating sodium channels. With $K J=1000$, the sodium activation function rises rapidly when the voltage is around $-50 \mathrm{mV}$.

To fix the problem above, both $p$ and $K J$ should be increased. To reduce the spike detection uncertainty, the cooperative gating sodium activation functions should rise at a smaller voltage near the spike initiation voltage. Also, the ratio parameter should be high enough such that the slow rising dynamics from the standard sodium channel have less influence on the AP initiation. However, the model provided in the code, which has no cooperative gating of the sodium inactivation, may not generate spikes properly when $p$ and $K J$ take larger values. Fig 46 is an illustration of this problem. Fixing $K J=1000$, I increased the ratio of cooperative gating sodium channels to $20 \%$. With the stochastic stimulus that generates the linear response curves in Fig $43 \mathrm{~B}$, the neuron model will stop generating spikes and have its voltage blocked in some intermediate value. The blocked voltage is caused by the large upward fluctuations in the stimulus. The inactivation of the sodium channels will be deinactivated at a larger voltage when responding to a large amplitude input. The early deinactivation will trigger more sodium current which may prevent the potassium current bringing down the voltage. Instead, the extra sodium current will balance with the potassium current at some intermediate voltage. For neuron models with cooperative gating sodium channels, it is more likely to happen. Stronger cooperativity of the sodium channels indicates that the sodium current will have a step change at smaller voltage. With the same sodium inactivation dynamics, there will be more sodium current during the deinactivation. Fig 47 provides an illustration of this phenomenon. The time 
step is set to be $0.001 \mathrm{~ms}$. Injecting a slightly larger constant input to the cooperative gating model, the inactivation variable of the sodium channels rises faster near $-50 \mathrm{mV}$. Previously, I have shown that for $K J=1000$, the cooperative gating sodium activation function rises instantaneously at $-50 \mathrm{mV}$. The slight difference of the inactivation dynamics leads to the block of spike generation.

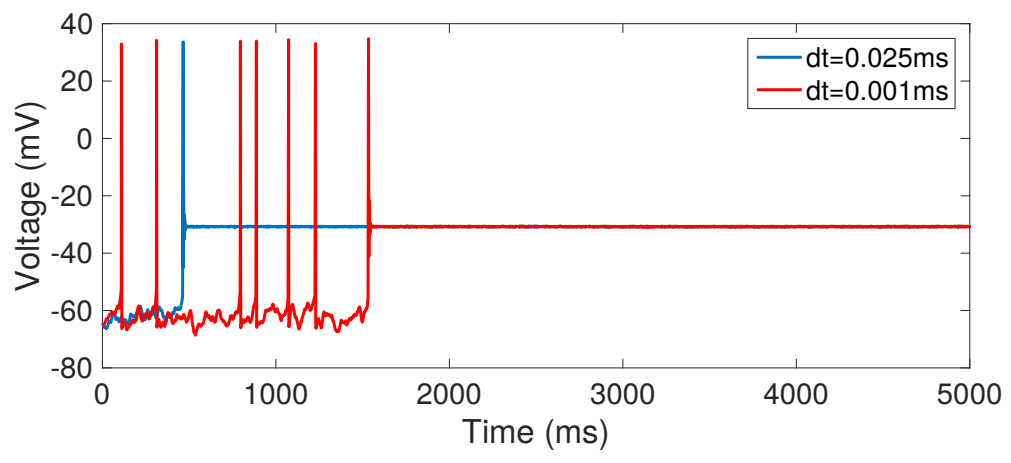

Fig 46. With a higher ratio of cooperative gating sodium channels, the firing behaviors are blocked by large stimulus fluctuations. Setting $p=0.2, K J=1000$, with the stimulus that generates the linear response curves in Fig $43 \mathrm{~B}$, the spiking behaviors are blocked. Reducing the time step from $0.025 \mathrm{~ms}$ to $0.001 \mathrm{~ms}$ doesn't resolve this problem.
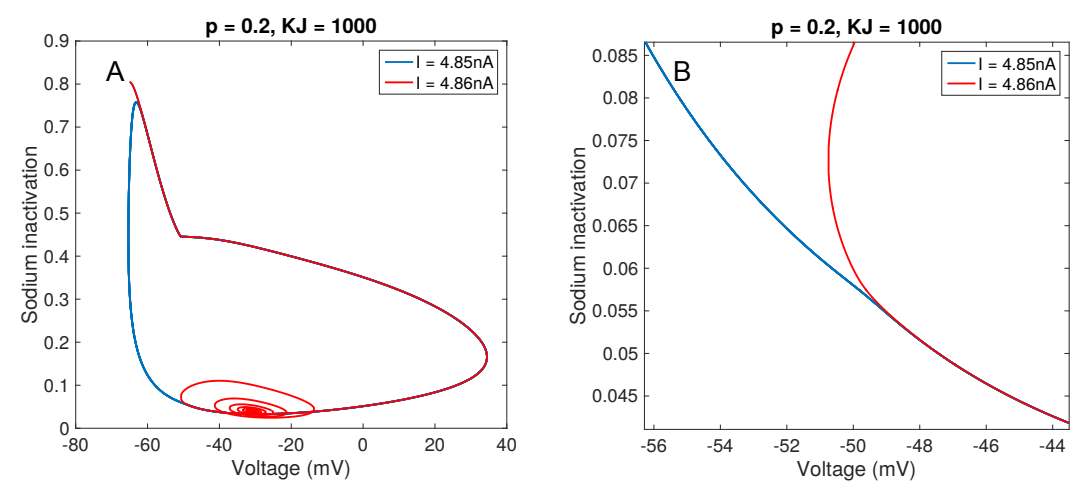

Fig 47. The sodium channels are deinactivated earlier when the stimulus amplitude is larger, which leads to the block of spiking behavior. With $p=0.2, K J=1000$, the neuron model is injected with constant inputs. Here $\mathrm{dt}=0.001 \mathrm{~ms}$. Slightly increasing the constant input makes the sodium inactivation variable rise earlier, which leads to the block of the spike generation. Figure B is a zoom-in of figure A near $-50 \mathrm{mV}$.

To make the neuron fire properly, it requires the sodium channels to be inactivated until the voltage is low enough. One simple solution is to introduce cooperative gating of the sodium inactivation. The expressions are given by Eqn 45 to 47. Note here we can choose a different coupling strength $K_{h} J_{h}$ from that of the sodium activation. Based on the equations of cooperative gating of sodium inactivation, during the activation of sodium channels, the inactivation variable will decrease earlier. During the deinavtivation of sodium channels, the cooperative gating inactivation 
variable will take a smaller value compared to that of the standard sodium inactivation variable. Fig 48 shows the dynamics of cooperative gating sodium inactivation variable. The coupling strength $K J$ of the sodium activation is 8000 . The coupling strength $K_{h} J_{h}$ of the sodium inactivation is also 8000 . The ratio of sodium channels that have both cooperative gating of sodium activation and inactivation is 0.7 . The neuron model is injected with a stochastic stimulus to reproduce $5 \mathrm{~Hz}$ firing rate and $\mathrm{CV}$ of ISI about 0.85 . The correlation time of the stimulus is $50 \mathrm{~ms}$. The behavior of inactivation variable fits with the deduction based on the equation.

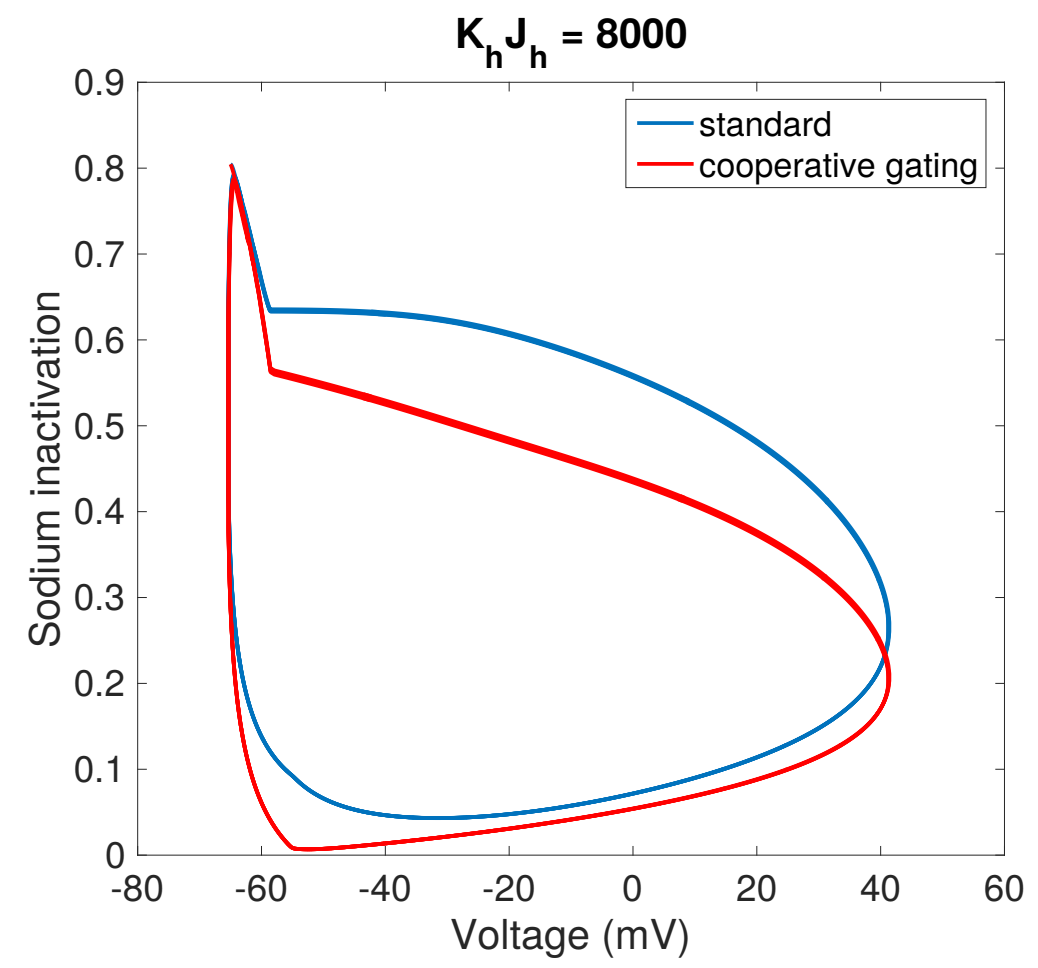

Fig 48. Cooperative gating of the sodium inactivation variable. With $K_{h} J_{h}=8000$, the inactivation variable drops to a lower value during the sodium activation. The inactivation variable will also be smaller during the deinactivation of sodium channels. The sodium channels are deinactivated instantaneously at a low enough voltage, so that the neuron can generate spike properly with strong cooperative gating of sodium activation dynamics.

Up till now, I have fixed the problem of spike generation of the neuron model with strong cooperative gating of sodium activation. However, this still doesn't resolve the problem of reproducing fast population encoding. In Fig $49 \mathrm{~A}$, the parameter $K J$ is increased to 15000 . The ratio parameter $p$ is set to 1 . To make the neuron fire, the parameter $K_{h} J_{h}$ is set to 30000 . With such strong cooperative gating, the encoding ability in the high frequency regime are much more improved compared with the original WB model. In the high frequency limit, the linear response curves become flat which reflect that the AP activation dynamics are similar to that of LIF model. Even so, the neuron model still fails to reproduce a high cutoff frequency in its linear response functions. Increasing the correlation time of the input shares the similar features with the WB model. 

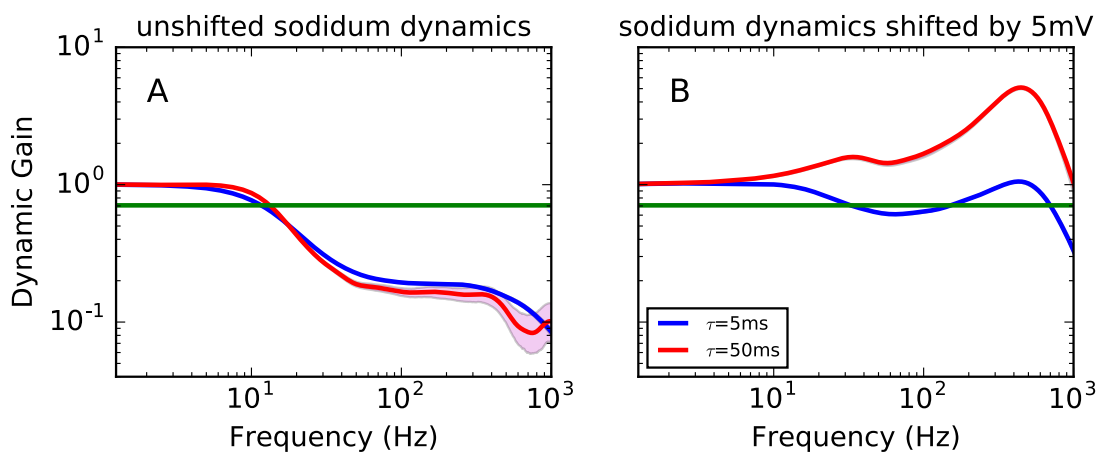

Fig 49. Linear response functions of the neuron models with strong cooperative gating of sodium channels. The coupling parameter for the sodium activation $K J$ is set to 15000 . To make the neuron model fire properly, the coupling parameter of the sodium inactivation $K_{h} J_{h}$ is set to 30000 . All sodium channels are of cooperative gating channels. A: with strong cooperative gating of sodium activation, the encoding ability is greatly enhanced compared to the original WB model. However, the linear response functions are still not sensitive to the correlation time of inputs. The firing rate is about $5 \mathrm{~Hz}$. The CV of ISI is about 0.85 . B. Shifting the sodium activation and inactivation dynamics by $5 \mathrm{mV}$ to the positive direction, both high bandwidth encoding and sensitivity of the dynamic gain to the correlation time are reproduced. The firing rate is about $5 \mathrm{~Hz}$. The $\mathrm{CV}$ of ISI is about 1 for $\tau=5 \mathrm{~ms}$ and about 1.5 for $\tau=50 \mathrm{~ms}$.
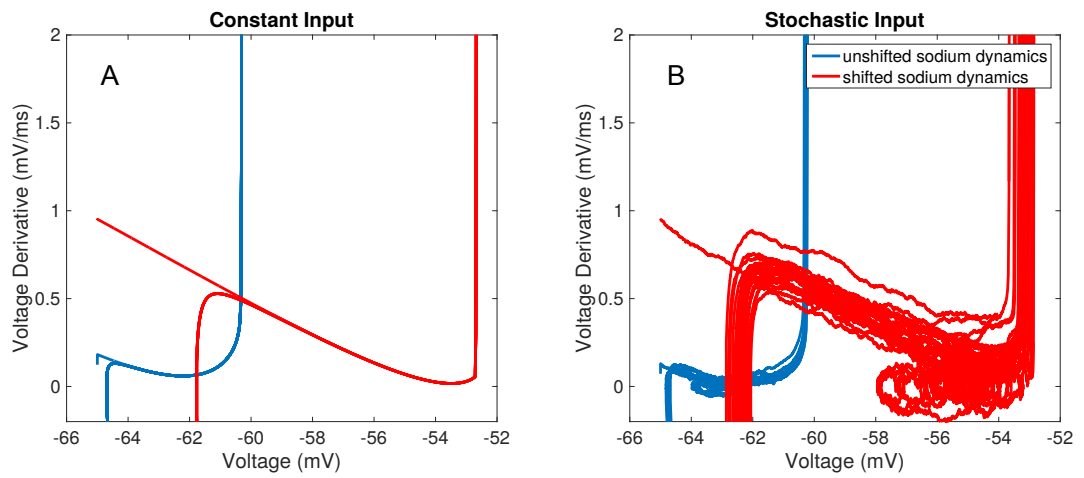

Fig 50. Phase plot at spike initiation of the cooperative gating model with and without shifting the sodium dynamics. The parameters are the same as those in Fig 49, A: the neuron models are injected with constant inputs of $5 \mathrm{~Hz}$ firing rate. B: the neuron models are injected with stochastic inputs for calculating the linear response function. The correlation time of the input is $50 \mathrm{~ms}$. The simulation time is $1 \mathrm{~s}$. The time step is $0.001 \mathrm{~ms}$. For the neuron model with shifted sodium activation dynamics, burst firing was detected in the simulation. So the spikes recorded are more than those without shifting the sodium dynamics.

With the knowledge of uncertainty in spike detection delay, we know that high cooperative gating doesn't really remove the slow activation dynamics after the spike threshold. Strong cooperative gating of sodium activation does make the activation function have an instantaneous rise when reaching 
some voltage. By the same time, it also increases the sodium activation probability at lower voltage values. This may make the spike initiated at a lower voltage, which does not effectively remove the slow AP initiation. Two methods can solve this problem. One is to suppress the slow sodium activation at the low voltage such that the collective open probability of the sodium channels in low voltage regime is smaller than independent sodium channels. In this way we remove the slow initiation part of the sodium activation. The other is to shift the sodium activation and inactivation dynamics in the positive direction along the voltage axis. At higher voltages, there will be more leak and potassium current to overcome before spike generation. The slope of potassium activation curves will also increase with the voltage. To change the slope of voltage derivative from negative to positive, it requires more sodium current which may have a more rapid activation dynamics. Fig $49 \mathrm{~B}$ shows the impact of shifting sodium dynamics on population encoding. Here it not only reproduces the properties of fast population encoding, but also displays a resonance peak in high frequency regime. This is because shifting the sodium dynamics also changes the AP generation mechanisms, which makes the neuron model more likely to have burst firing. Even with a small std stimulus, the CV of the ISI can already reach 1 when the correlation time is $5 \mathrm{~ms}$. For larger correlation time $50 \mathrm{~ms}$, the burst firing feature is more significant. This makes it difficult to search for the stimulus parameters to reproduce the same CV in Fig 49A. So here I only control the firing rate to be $5 \mathrm{~Hz}$. In the next chapter, I will show that shifting the sodium dynamics will change the neuron model from type I to type II. There will be a high discontinuity in the neuron model's F-I curve, which leads to burst firing.

In the last part of this section, I will examine the uncertainty of the spike detection directly for these two models. In Fig 50. I plotted the phase plots of the cooperative gating model with and without shifting the sodium dynamics. The phase plots focus on AP initiation. In A, the phase plots are generated with the constant inputs for $5 \mathrm{~Hz}$ firing rate. In $\mathrm{B}$, the phase plots are generated with the stochastic inputs used in Fig 49 . The correlation time is $50 \mathrm{~ms}$. In the method chapter, we know that the original WB has a spike initiation voltage at $-60 \mathrm{mV}$. The spike detection delay is mainly happened in the first $10 \mathrm{mV}$ after the spike initiation. It can be expected that this delay will be shorter if the AP initiation is more rapid. The std of spike detection delay in WB model is approximately $20 \mathrm{~ms}$ when the correlation time is equal to $50 \mathrm{~ms}$. Here with strong cooperative gating of sodium activation, the spike initiation voltage is at about $-62.26 \mathrm{mV}$. From the phase plot in A we can see that the rapid onset is at $-60 \mathrm{mV}$. The current realization of the cooperative gating keeps the slow AP initiation. In comparison, the spike initiation voltage for the model with shifted sodium dynamics is $-53.48 \mathrm{mV}$. The slow initiation part is shortened. This property is also kept with the stochastic input. I also estimated the std of spike detection delay numerically for these two models with the stimuli I used for calculating the linear response functions. The estimation is based on the spikes generated in 20s simulation, shown in Fig 32 . For the model without shifting the sodium dynamics, the spike detection voltage is at $-16 \mathrm{mV}$. The std of the spike detection delay is about $18.57 \mathrm{~ms}$, which is similar to the original WB model. For the model with shifted sodium activation, the spike detection voltage is at $-38 \mathrm{mV}$. The std of the spike detection delay is only about $4.50 \mathrm{~ms}$. The spike detection voltages in these models are the voltages that have the maximum voltage derivative, so the estimated std values are 
not affected by too small spike detection voltages.

\subsection{The Impact of AP Initiation Site Location and Soma Size on Population Encoding}

In previous chapter, I failed to see the impact of AP initiation site on encoding ability of Brette's model. One may wonder whether this parameter has a contribution to population encoding. If it does, then in which condition can we observe its impact? From the phase plots of the AP generation dynamics, we have already known that separating the AP initiation site further away from the soma can reduce the lateral current entering the soma during AP generation. When the neuron model has a slow sodium activation function, the encoding ability is undermined by the slow sodium activation dynamics. A change of the lateral current in this condition does not have a big impact on the AP initiation dynamics. In the other extreme condition, the sodium activation function is designed like a step function. The major role of separating AP from the soma is filtering high frequency components of the stochastic stimulus. The high frequency components are more damped out compared with the low frequency components. However, when calculating the linear response function, the Fourier transform of the STA is divided by the power spectral density of the injected current in the soma rather than that of the current received by the AP initiation site. So the linear response functions in this condition are similar to those of the LIF model in the low frequency regime. In the high frequency regime, the gain curves have a decay rather than remain flat. So what will happen if we design a sodium activation function that both remove slow AP initiation and have a not too high activation slope? Removing slow AP initiation reduces the major resource of uncertainty in spike detection. A not too high sodium activation slope can let the lateral current make a contribution to the rapidness of spike generation. With these two criteria satisfied, we can see the impact of AP initiation site on the encoding ability.

The further implication of this sodium activation function design is to highlight the importance of removing slow sodium initiation. Previous design of onset rapidness focused on the slope at some specific point on the phase plot or the I-V curve. These points are usually far away from the spike initiation voltage. When the high bandwidth encoding is realized, the slope at this point is extremely high. Here I will show that with the condition of removing slow initiation fulfilled, the high bandwidth encoding can be realized without too high slope at the middle point of activation function as we previously measured.

To design a sodium activation function that both without slow activation and with a not too high activation slope, I implement a piece-wise linear function described as:

$$
m_{\infty}\left(V_{a}\right)= \begin{cases}0 & \text { if } V_{a}<-50 \mathrm{mV} \\ \frac{1}{40}\left(V_{a}+50\right) & \text { if }-50 \mathrm{mV} \leqslant V_{a} \leqslant-10 \mathrm{mV} \\ 1 & \text { if } V_{a}>-10 \mathrm{mV}\end{cases}
$$

The activation part has a constant slope of $1 / 40$. Compared with the standard sodium activation function used in Brette's model, which has a slope of $1 / 24$ at the middle point with $k_{a}=6 \mathrm{mV}$, the current sodium activation function seems to be slower. However, near the spike initiation voltage, the AP generation dynamics is much more rapid for this piece-wise linear function. 

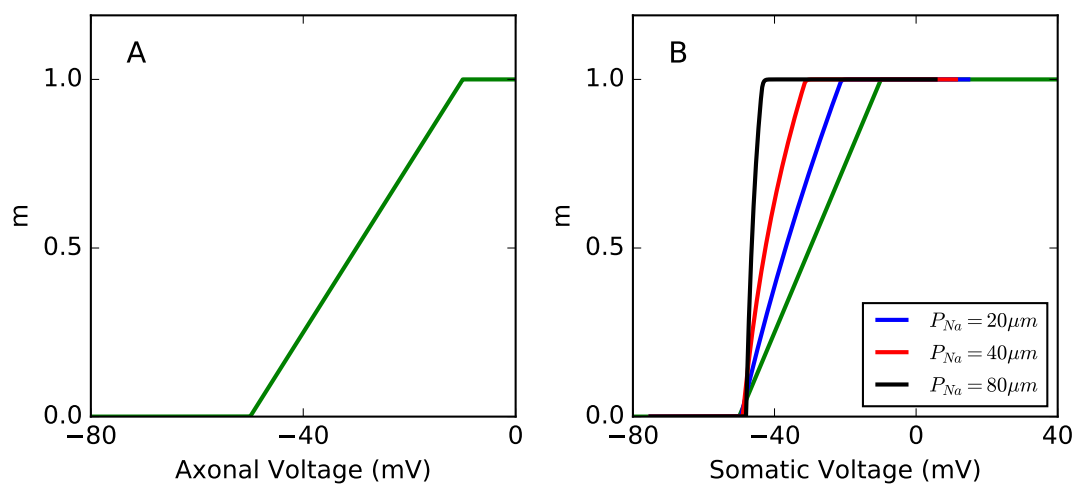

Fig 51. Piece-wise linear sodium activation curve versus axonal and somatic voltages. A: The sodium activation variable $m$ is 0 below $-50 \mathrm{mV} . \mathrm{m}$ is 1 above $-10 \mathrm{mV}$. The sodium activation function in between is linear. B: Represented as the function of somatic voltage, the sodium activation function has a higher activation slope for a larger separation of AP initiation site. The voltage from which the activation function starts to rise is slightly delayed.

Fig 51 shows the sodium activation function and its representation as the function of somatic voltage with different AP initiation locations. Similar to the representation of standard sodium activation function in Brette's model, the activation dynamics seen from the soma becomes more rapid when the AP initiation separation is larger. Also, because of the suppression of the sodium current at the low voltage, the new representations keep the feature of sodium current suppression. The representations of the piece-wise linear function also share a feature of representing step-like function. For larger separation distances, the voltages that the sodium current rise from zero are delayed. As a summary, the sodium activation function representations are more similar to the step-like functions. From the view of writing the axonal sodium activation function into the somatic voltage equation, the AP initiation dynamics become more rapid.

In Fig 52, I compare spike shapes and phase plots of neuron models equipped with piece-wise linear sodium activation function. Seen from the AP initiation site, the spike shapes become sharper when the AP initiation site is further away. However seen from the soma, there is no such significant difference. The spike initiation part is slightly less smooth when $P_{N a}=80 \mu \mathrm{m}$. In the aspect of phase plots of the axonal voltage, we can see a clear impact of lateral current on the AP onset dynamics. The lateral current does not change the voltage where the spike is initiated. All three curves rise around $-50 \mathrm{mV}$. But for a larger separation of the AP initiation site, there is less lateral current entering the soma during spike generation. As a result, the spike generation speeds are clearly faster in corresponding models. Seen from the soma, the somatic voltage also rises more rapidly for a larger $P_{N a}$. The observations above are also valid when injecting stochastic stimuli into the soma.

Through calculating the linear response functions directly, we can see that with a piece-wise linear sodium activation function, the encoding ability in the high frequency regime is enhanced when the AP initiation site is separated further from the soma. Besides, for all three positions of the AP initiation sites, the encoding ability is improved for larger correlation 

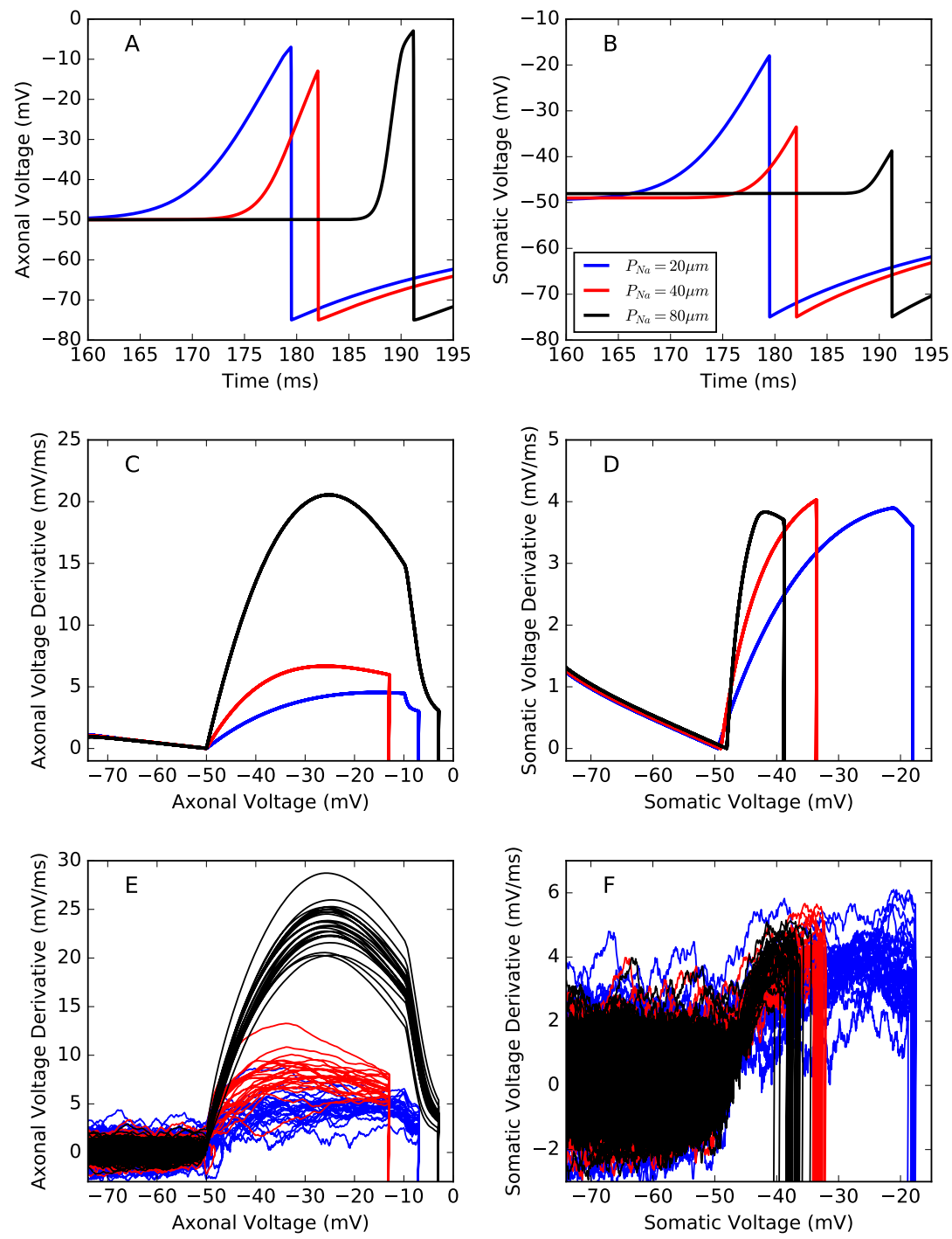

Fig 52. Sharper spike onset dynamics at the soma and the axon for larger separations of AP initiation site. A, B: The spike shapes under constant inputs triggering a firing rate around $5 \mathrm{~Hz}$ in three conditions separately. C, D: The phase plots of the spiking voltages under the constant inputs. The drastic drop of the voltage derivative at the end of each curve corresponds to the negative voltage derivative at the voltage reset. E, F: The phase plots of the spiking voltages under the stochastic inputs triggering a firing rate of about $5 \mathrm{~Hz}$. The $\mathrm{CV}$ of inter spikes intervals is $0.85 \pm 0.05$. Correlation time of the input is $5 \mathrm{~ms}$. All the figures share the same legend in B.

time of the input. For $P_{N a}=80 \mu m$, the linear response function has a cutoff frequency of about $200 \mathrm{~Hz}$ when the correlation time of the input is $50 \mathrm{~ms}$. It can be expected that further increasing the slope of the piece-wise linear activation function will improve the encoding ability for all three AP initiation sites. But the impact of the AP initiation sites on the encoding will begin to wane. This implies that the phenomenon in Fig 53 could only 

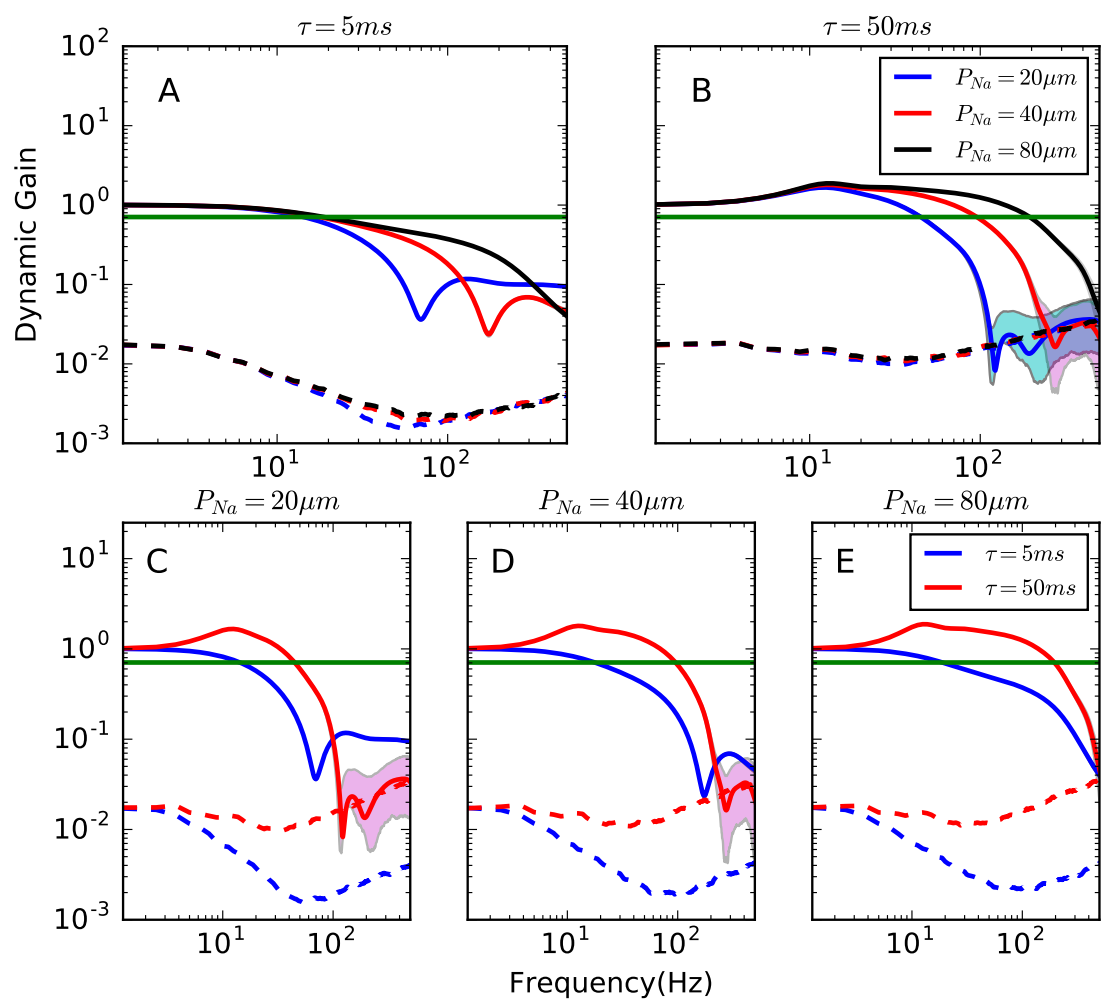

Fig 53. Linear response functions of neuron models with a piece-wise linear sodium activation curve. All the denotations are the same as those in the previous chapter. In this case, increasing the separation distance of AP initiation site enhances the encoding ability of neuron models. Both high bandwidth encoding and sensitivity of the dynamic gain to the correlation time of the background current are reproduced.

be observed when the lateral current size is comparable to the AP initiation current. If the sodium activation slope is larger, or the sodium conductance is much higher, then the encoding ability would be similar to that of the LIF like model. The filtering effect on the stimulus when transmitting from the soma to the AP initiation site may have a larger influence on the dynamic gain.

In the last part, I will examine the impact of soma size on population encoding. In previous chapter, with the simplified model, I have shown that the linear response function is most sensitive to the diameter of the soma. But this is based on the assumption that other parameters are fixed. In simulation, I controlled the firing conditions across different models to evaluate the encoding ability. Here I will apply this criterion to check the impact of soma size on the encoding. I calculated the linear response functions of the neuron model with a small soma. The sodium activation is the step-like function with $k_{a}=0.1 \mathrm{mV}$. The soma size is decreased to $10 \mu \mathrm{m}$ from $50 \mu \mathrm{m}$. The major difference between these two types of models is the size of the lateral current. With a smaller soma, the lateral current is smaller, which makes the AP initiation dynamics reach a higher maximum voltage derivative. The difference in the lateral current has limited effect on the uncertainty of spike time detection. As a result, we can see that the 

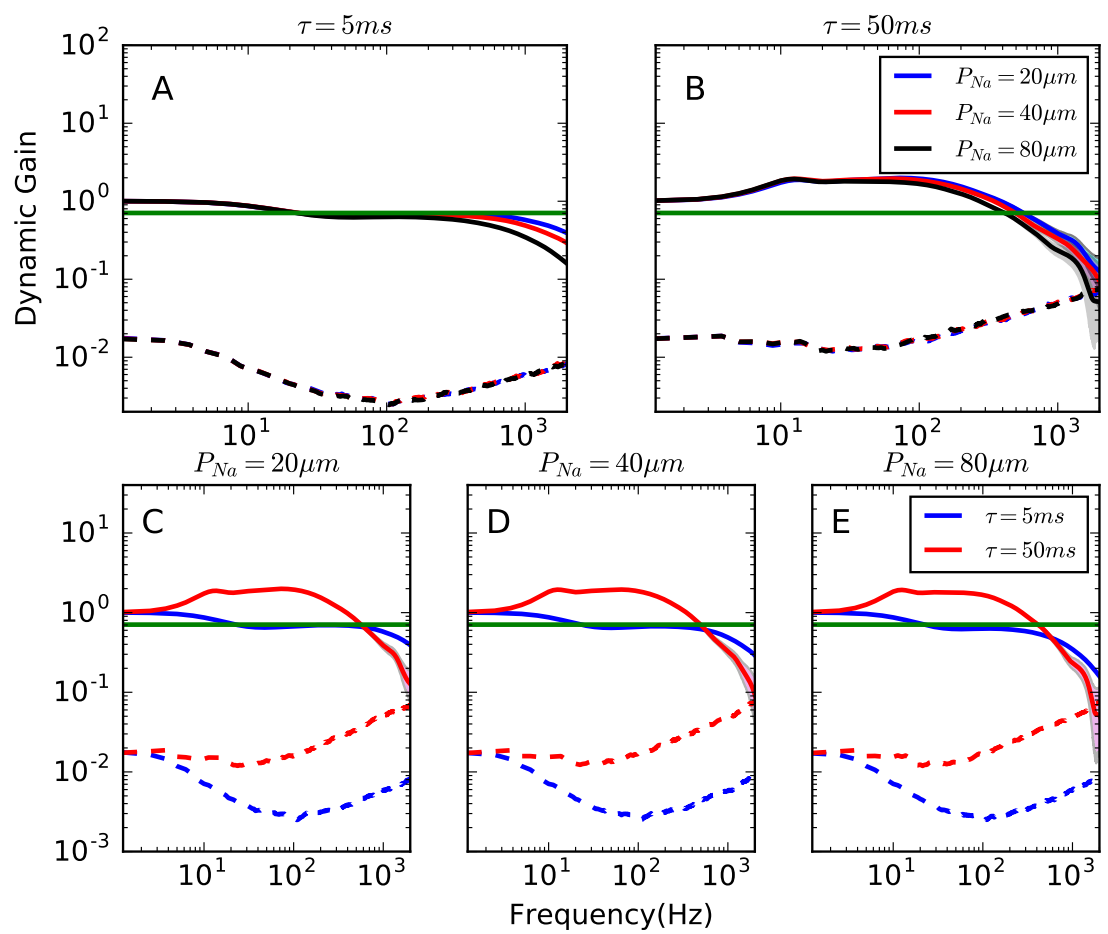

Fig 54. Linear response of the neuron model with the step-like sodium activation curve and a small soma. All the denotations are the same as with the previous chapter. The sodium activation parameter $k_{a}$ is $0.1 \mathrm{mV}$. The diameter of the soma is set to $10 \mu \mathrm{m}$. The encoding ability is further enhanced compared with the model having a big soma.

linear response functions are better approximations of the LIF model. The dynamic gain decay in the high frequency limit are similar to that of the big soma model. 


\section{High Bandwidth Encoding with High Fre- quency Repetitive Firing}

In the introduction chapter, I introduced the proposal that a large dendrite can enhance the population encoding ability of the neuron model [49]. One interesting observation of the neuron model implemented in this work is that even without the dendrite part, the neuron model can already realize a cutoff frequency above $100 \mathrm{~Hz}$ in its linear response function. Here the neuron model doesn't require rapid AP onset. No cooperative gating of the sodium channels are assumed. These results seem to indicate that high bandwidth encoding can be realized without rapid AP onset. So one may wonder, what are the underlying mechanisms that realize high bandwidth encoding? Furthermore, is the dynamic gain in the high frequency regime sensitive to the correlation time of the input? If so, it implies that fast population encoding can be realized without explicit restrictions on spike onset rapidness or cooperative gating of sodium channels.

In this chapter, I will look into this neuron model and provide an explanation for the high bandwidth encoding. First, I will give a more detailed introduction to the neuron model used in [49] and reproduce high bandwidth encoding in its linear response functions. Second, I will show that the high bandwidth encoding is realized by high frequency repetitive firing. The F-I curve of the neuron model has a high discontinuity at the threshold where the neuron begins to generate spikes. This implies that the neuron is more likely to generate a burst of spikes in a short time interval, which results in a high CV of ISI. The high frequency repetitive firing enhances the dynamic gain at corresponding frequencies in the linear response function and helps to realize the high cutoff frequency. Third, I will show that the multi-compartment framework and the relative position of the sodium and potassium activation dynamics on the voltage axis are the two major reasons for high frequency repetitive firing. Shifting the sodium activation function towards the positive direction in the voltage axis will change the neuron model from a type I model to a type II model. This will lead to the high discontinuity in the F-I curve.

\subsection{Introduction to Eyal's Model}

In this section, I will first give an introduction to the multi-compartment model used in [49. Here I only consider the model without the dendrite. Fig 55 is a representation of the morphology of the multi-compartment model adapted from [49, which is first proposed in 92, 93. The neuron model is composed of a soma, an axon initial segment(AIS) and a myelinated axon. The soma is a cylinder of length $30 \mu \mathrm{m}$. The diameter of the soma is $20 \mu \mathrm{m}$. The AIS is of length $50 \mu \mathrm{m}$. The diameter of AIS is $1 \mu \mathrm{m}$. The myelinated axon is of length $1000 \mu \mathrm{m}$. The diameter of myelinated axon is $1 \mu \mathrm{m}$. During the simulation, the somatic voltage is recorded in the middle of soma. The axonal voltage is recorded $47 \mu \mathrm{m}$ from the soma in the AIS. Sodium and potassium channels are distributed in the soma and the AIS. The myelinated axon is passive and has much smaller specific leak conductance and specific capacitance than that of the soma and the AIS. The specific leak conductance at the soma and the AIS is $3.3 \times 10^{-5} \mathrm{~S} / \mathrm{cm}^{2}$. The specific leak conductance at the myelinated axon is $6.6 \times 10^{-7} \mathrm{~S} / \mathrm{cm}^{2}$. The balanced voltage for the leak $E_{\text {pas }}$ is $-70 \mathrm{mV}$. The specific capacitance in the soma and the AIS is $0.75 \mu \mathrm{F} / \mathrm{cm}^{2}$. The specific capacitance in the myelinated axon is 
$0.02 \mu \mathrm{F} / \mathrm{cm}^{2}$. The longitude resistance for all compartments is $100 \Omega \cdot \mathrm{cm}$. The sodium current density is described as:

$$
i_{N a}=\operatorname{tadj} \cdot g_{N a} \cdot m^{3} h \cdot\left(V-E_{N a}\right)
$$

tadj is a parameter associated with the temperature, which is described as $2.3^{(37-T e m p) / 10}$. The temperature Temp is fixed to be 23 degrees. $g_{N a}$ is the specific sodium conductance, which is set to $800 \mathrm{pS} / \mu \mathrm{m}^{2}$ in the soma. In the AIS, the specific sodium conductance is $8000 p S / \mu m^{2} . m$ is the activation variable of sodium channels. $h$ is the inactivation variable of sodium channels. $E_{N a}$ is the balanced voltage equal to $50 \mathrm{mV}$. For the activation variable $m$, it has the equation:

$$
\tau_{m} \frac{d m}{d t}=m_{\infty}-m
$$

where:

$$
\begin{gathered}
\tau_{m}=\frac{1}{\operatorname{tadj}(a+b)} \\
m_{\infty}=\frac{a}{a+b} \\
a=R_{a} q_{a} \frac{\left(t h_{a}-V\right) / q_{a}}{\exp \left(\left(t h_{a}-V\right) / q_{a}\right)-1} \\
b=R_{b} q_{a} \frac{\left(V-t h_{a}\right) / q_{a}}{\exp \left(\left(V-t h_{a}\right) / q_{a}\right)-1}
\end{gathered}
$$

Here $R_{a}$ is $0.182 \mathrm{~ms}^{-1}$. $R_{b}$ is $0.124 \mathrm{~ms}^{-1}$. $q_{a}$ describes the activation slope, which is set to $9 \mathrm{mV}$. $t h_{a}$ is $-25 \mathrm{mV}$.

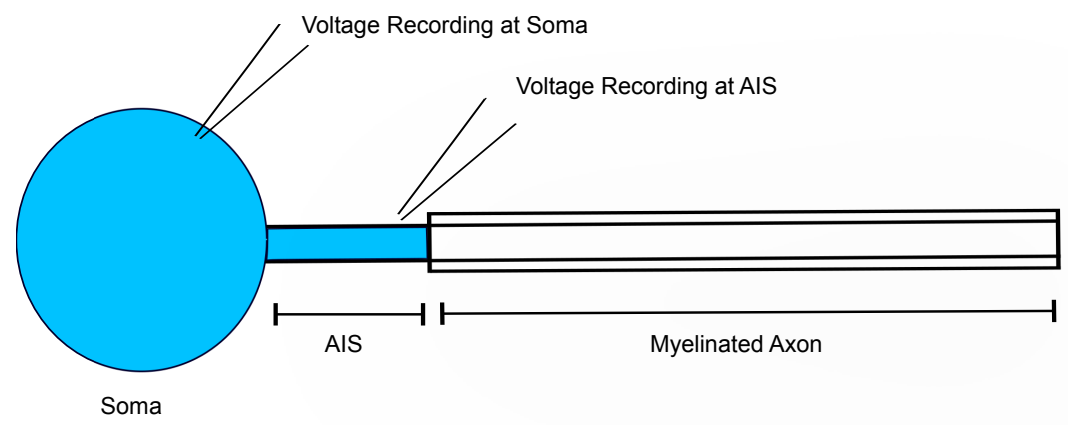

Fig 55. Neuron morphology of Eyal's model. The neuron model is composed of a soma, an axon initial segment and an myelinated axon. The ion channels are distributed in the soma and the axon initial segment, which are the blue regions in the model. The figure is adapted from the neuron morphology in 49].

The inactivation variable $h$ has the equation:

$$
\tau_{h} \frac{d h}{d t}=h_{\infty}-h
$$

where:

$$
\begin{gathered}
\tau_{h}=\frac{1}{\operatorname{tadj}\left(a_{h}+b_{h}\right)} \\
h_{\infty}=\frac{1}{1+\exp \left(\left(V-t h_{i n f}\right) / q_{i n f}\right)}
\end{gathered}
$$




$$
\begin{aligned}
& a_{h}=R_{d} q_{i} \frac{\left(t h_{i 1}-V\right) / q_{i}}{\exp \left(\left(t h_{i 1}-V\right) / q_{i}\right)-1} \\
& b_{h}=R_{g} q_{i} \frac{\left(V-t h_{i 2}\right) / q_{i}}{\exp \left(\left(V-t h_{i 2}\right) / q_{i}\right)-1}
\end{aligned}
$$

Here $R_{d}$ is $0.024 \mathrm{~ms}^{-1}$. $R_{g}$ is $0.0091 \mathrm{~ms}^{-1} . q_{i}$ is $5 \mathrm{mV} . t h_{i 1}$ is $-40 \mathrm{mV}$. $t h_{i 2}$ is $-65 \mathrm{mV}$. $t h_{\text {inf }}$ is $-55 \mathrm{mV}$. $q_{\text {inf }}$ is $6.2 \mathrm{mV}$.

The potassium current density is:

$$
i_{K}=\operatorname{tadj} \cdot \bar{g}_{K} \cdot n \cdot\left(V-E_{K}\right)
$$

tadj is the same as above. The specific potassium conductance $\bar{g}_{K}$ is $320 \mathrm{pS} / \mu \mathrm{m}^{2}$ at the soma, and $1500 \mathrm{pS} / \mu \mathrm{m}^{2}$ at the AIS. $E_{K}$ is $-85 \mathrm{mV}$. The potassium activation variable $n$ has the equations:

$$
\tau_{n} \frac{d n}{d t}=n_{\infty}-n
$$

where:

$$
\begin{gathered}
\tau_{n}=\frac{1}{\operatorname{tadj}\left(a_{n}+b_{n}\right)} \\
n_{\infty}=\frac{a_{n}}{a_{n}+b_{n}} \\
a_{n}=R_{a n} q_{a n} \frac{\left(t h_{a n}-V\right) / q_{a n}}{\exp \left(\left(h_{a n}-V\right) / q_{a n}\right)-1} \\
b_{n}=R_{b n} q_{a n} \frac{\left(V-t h_{a n}\right) / q_{a n}}{\exp \left(\left(V-t h_{a n}\right) / q_{a n}\right)-1}
\end{gathered}
$$

Here $R_{a n}$ is $0.02 m s^{-1}$. $R_{b n}$ is $0.002 m s^{-1}$. $q_{a n}$ describes the activation slope, which is set to $9 \mathrm{mV}$. $t h_{a n}$ is $25 \mathrm{mV}$.

Fig 56 provides the sodium activation dynamics $m^{3}$ of Eyal's model based on its parameters. As a comparison, I also plotted the sodium activation dynamics of Brette's model $m$. For both sodium activation curves, they share a similar activation speed. In Eyal's model, the sodium channels are activated at a larger voltage. Injecting the soma with a constant input, we can obtain the phase plot of spike generation dynamics shown in Fig 57. At the AIS, the axonal voltage derivative rises slowly with the voltage, which doesn't reproduce the rapid AP onset proposed in 7. Seen from the soma, the somatic voltage derivative rises faster than that in the AIS. Based on the criterion of AP onset rapidness proposed in [7], the slope of phase plot at $20 \mathrm{mV} / \mathrm{ms}$ doesn't have significant improvement in the soma than that in the axon.

To calculate the linear response functions of Eyal's model, I searched for the mean and the std of the stochastic stimulus to reproduce a firing rate of $5 \mathrm{~Hz}$ shown in Fig 58 . The mean-std relation is similar to those of the Brette's model and WB model. However, for the mean-CV relation, when the mean is getting larger, the CV of the ISI is not decreasing to zero. For three different correlation times of the inputs, it is not possible to find a common $\mathrm{CV}$ in the reasonable range. Later I will provide an explanation why the mean-CV relation is different from what we observed in previous chapters. So for this model, I fixed firing rate and mean of the stimulus when calculating the linear response function. Here the mean is chosen to be $0.03 \mathrm{nA}$. Fig 59 shows the linear response functions of Eyal's model. I successfully reproduced the high cutoff frequency of the linear response 


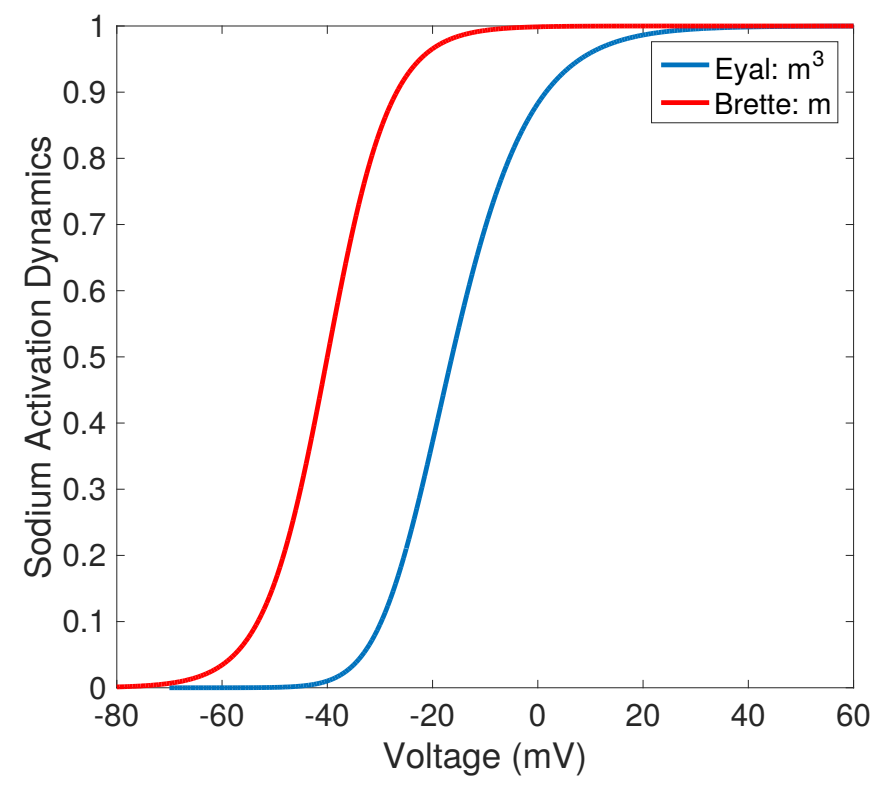

Fig 56. Sodium activation dynamics of Eyal's model. The blue curve is the sodium activation dynamics of the Eyal's model, which is $\mathrm{m}^{3}$. As a comparison, the red curve is the sodium activation dynamics of Brette's model, which is $m$. No rapid onset is included in both conditions.

functions when $\tau=5 \mathrm{~ms}$, which is given in 49 . The linear response function also has a resonance near $40 \mathrm{~Hz}$. However, increasing the correlation time of inputs doesn't enhance the encoding ability in the high frequency regime. Only higher resonance effect is obtained. This is contradictory with the observations in experimental studies 36 . So we can see that without rapid AP onset, Eyal's model only reproduces the high cutoff frequency in its linear response functions. The dynamic gain in the high frequency regime is not sensitive to the correlation time of inputs. Eyal's model is proposed to simulate the layer $2 / 3$ pyramidal neurons, but it doesn't capture the fast population encoding observed in pyramidal neurons.

It remains unclear about the underlying mechanisms that realize high bandwidth encoding in Eyal's model. In Fig 60, I plotted the F-I curve of Eyal's model. Different from the type I models we studied in the previous chapter, the F-I curve of Eyal's model has a high discontinuity. Once the constant input is across a threshold, the neuron model will generate a firing rate above $30 \mathrm{~Hz}$. This indicates that Eyal's model is a type II model. For a large mean and small std stimulus, the neuron will stay quiet when the stimulus is not enough to generate spikes. However, when the stimulus is slightly larger than the rheobase current, the neuron will generate a trial of high frequency repetitive firing in a short interval, which makes the CV of the ISI extremely high. For the type I neuron models we investigated before, the F-I curve is continuous with the constant input. The slope of F-I curve near the rheobase current is not high enough to realize high frequency firing with a short increase of the input current. The high frequency repetitive firing behavior explains the different mean-CV curve observed in Eyal's model.

Following this line of deduction, we can explain the resonance in linear 


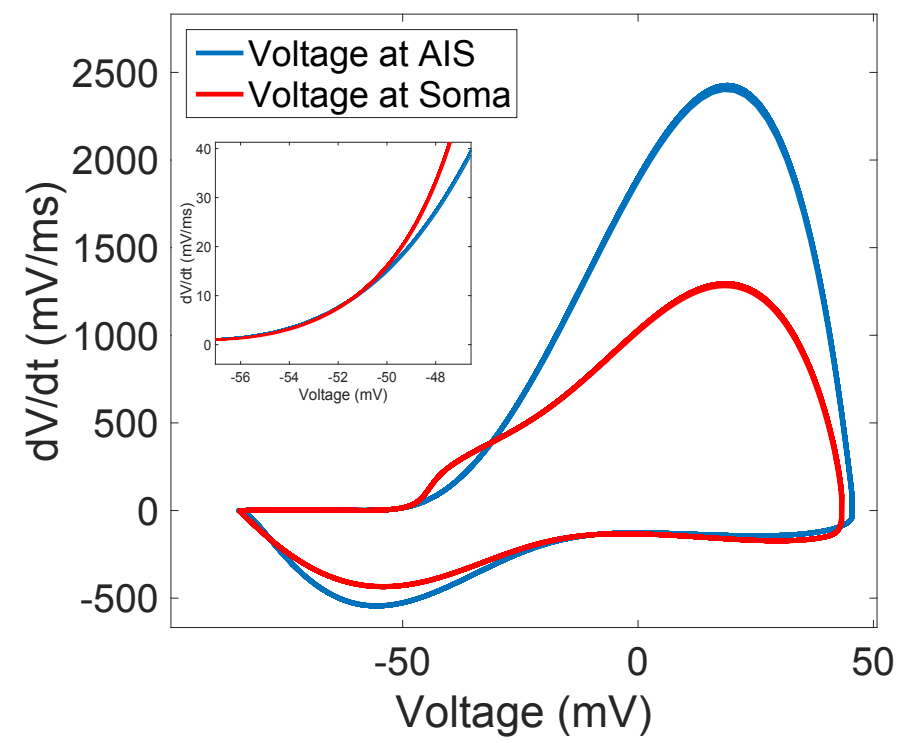

Fig 57. Phase plots of AP generation dynamics of Eyal's model. The neuron model is injected with a constant input. The voltage at the AIS are recorded $47 \mu \mathrm{m}$ from the soma. The voltage at soma is recorded in the middle of soma. The AP initiation dynamics are not rapid compared with the recordings in the cortical neurons 7 . The AP initiation dynamics at the soma are more rapid than that of the AIS.
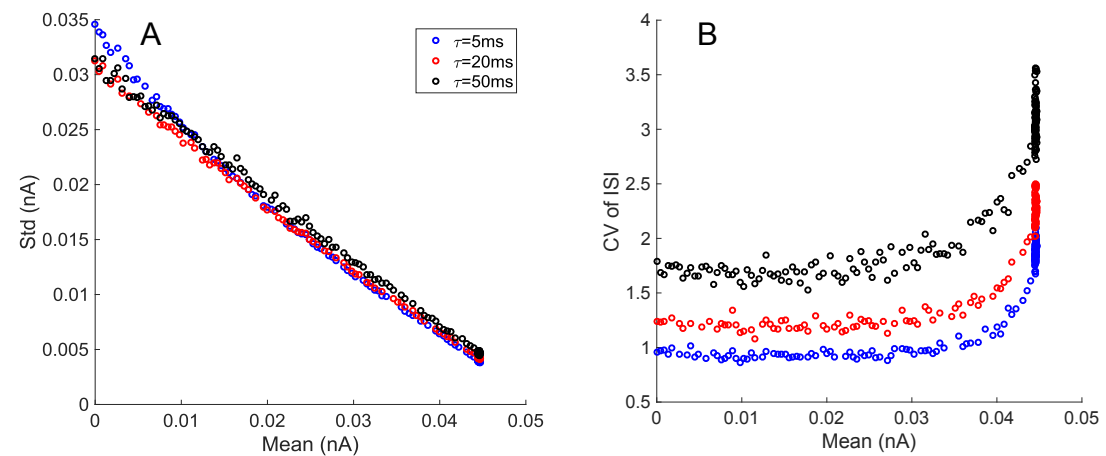

Fig 58. Mean-std and mean-CV relation of Eyal's model. In A, each pair of mean and std reproduces a firing rate of $5 \mathrm{~Hz}$ in Eyal's model. In $\mathrm{B}$, it plots the $\mathrm{CV}$ of ISI over the mean for each data point in $\mathrm{A}$.

response functions and the high bandwidth encoding. Since the neuron firing patterns are more likely to have pieces of high frequency burst firing, a large amount of the inter-spike intervals will fall in the range around the inverse of typical burst firing rate. From the view of vector strength method for calculating linear response functions, the amplitude of complex vector of typical burst firing rate will be selectively enhanced. For large mean small std stimulus, the frequency that are selective enhanced is the frequency near rheobase current in the F-I curve. If the enhanced frequency is high, it is possible to make the cutoff frequency of the linear response function much higher than the averaged firing rate without requiring rapid AP onset. 


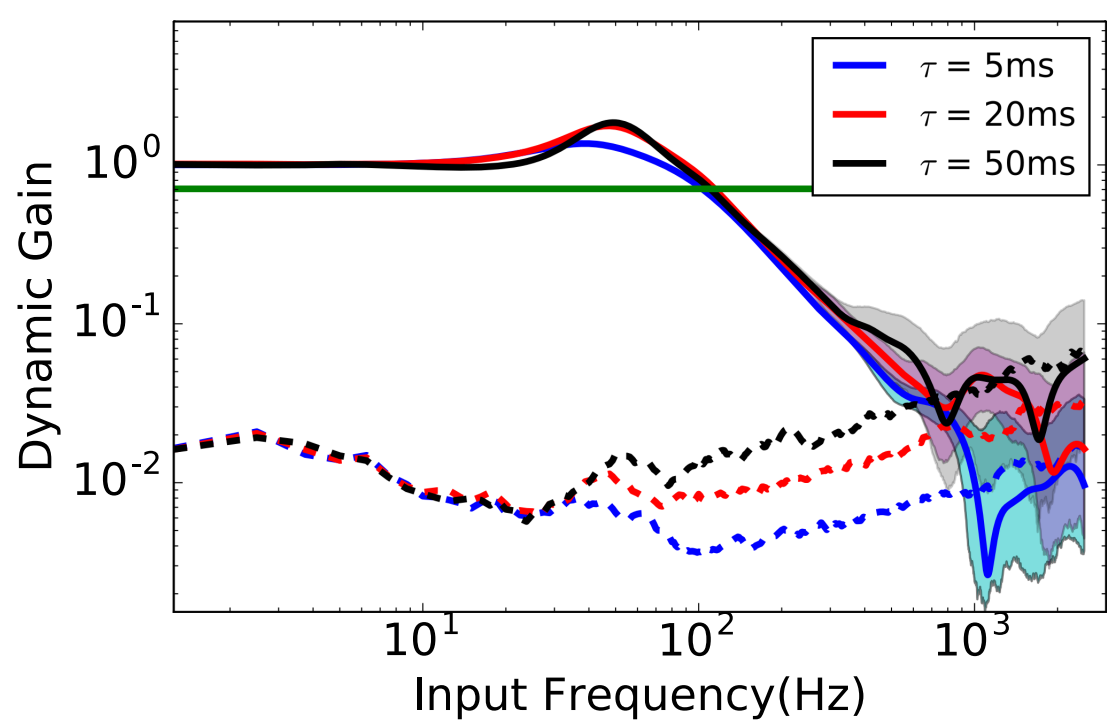

Fig 59. Linear response functions of Eyal's model. TBA.

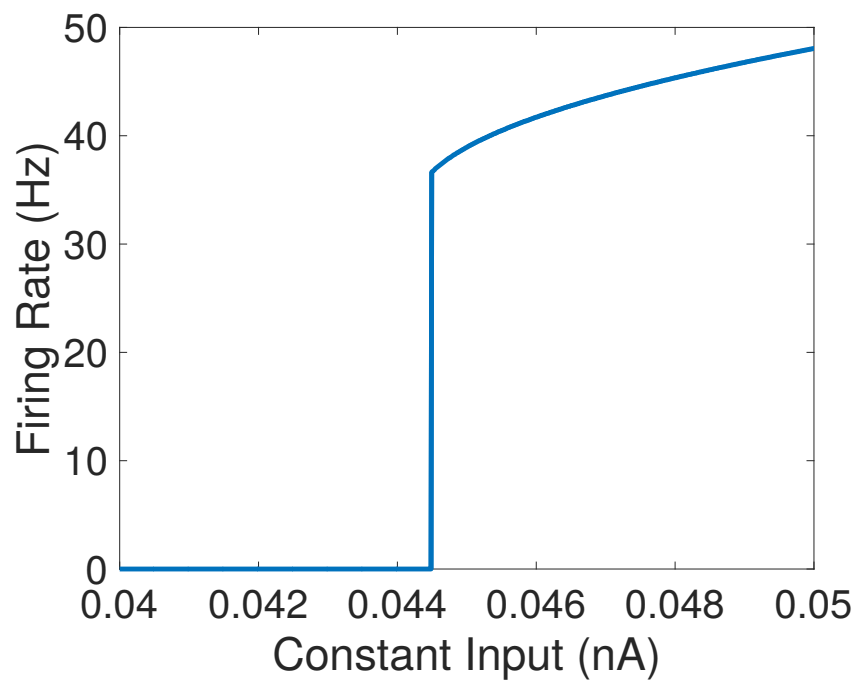

Fig 60. F-I curve of Eyal's model. The F-I curve has a big discontinuity at the rheobase current. This implies that the neuron model is a type II model. The neuron model is able to realize high frequency repetitive firing once the input is across the rheobase current.

For Eyal's model, the resonance frequency is around 40Hz. One simple way to examine this explanation is to plot the histogram of the inter-spike intervals. If the histogram has a strong peak at the inverse of the resonance frequency, then it indicates that the explanation is plausible. Fig 61 shows the histograms of ISI for different correlation times of the input. For a larger correlation time, the CV of ISI is larger. The histogram has a larger peak, where the peak of histogram fits with the resonance frequency in the linear response functions. 

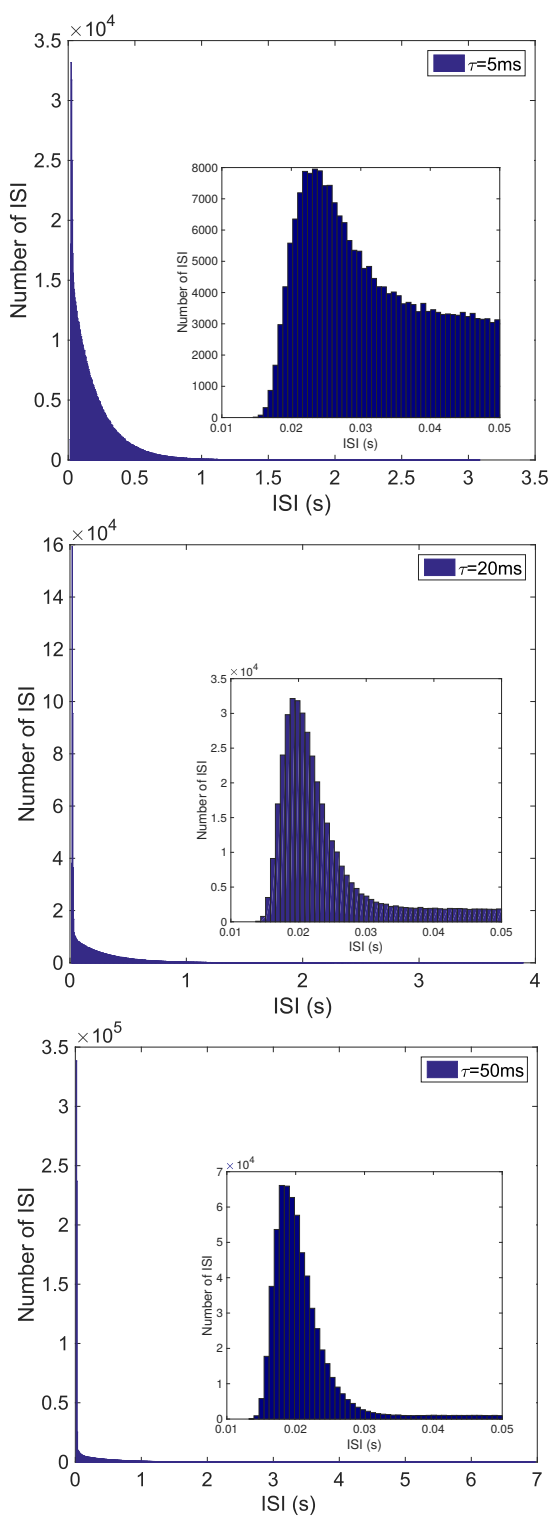

Fig 61. Histogram of the ISI for Eyal's model. For each correlation time, with the stimulus for calculating the linear response function, I generated one million ISI values for the histogram. For larger correlation times, the CV of the ISI is larger. The histogram has a larger peak at a smaller ISI value, which fits with the resonance frequency in the linear response function.

\subsection{The Mechanisms for High Frequency Repetitive Firing}

In previous section, I have shown that the high bandwidth encoding in Eyal's model is realized with high frequency repetitive firing. In this section, I will explore the underlying mechanisms for this phenomenon. As it can be seen that Eyal's model is a complicated multi-compartment model with ion channels distributed in soma and AIS. The ion channels includes both 
sodium and potassium channels which constitute a complete AP generation mechanism. In comparison, the WB model is only a single-compartment model. Also, Brette's model doesn't have complete AP generation dynamics. Brette's model needs to reset the voltage by hand. The simulation results in the previous chapters have shown that the original WB model and the Brette's model can't reproduce high bandwidth encoding. In the following work, by comparing with these two models, I will simplify Eyal's model step by step toward these two models in order to find out the mechanisms that are responsible for high bandwidth encoding.

First I will study the impact of multi-compartment framework on population encoding in Eyal's model. In Fig 62 A and B, I removed the ion channels in the soma of Eyal's model. The F-I curve in A shows that there is a smaller discontinuity in the firing rate when the neuron model begins to generate spikes. The neuron model is less capable of high frequency repetitive firing. As reflected in the linear response functions, when the correlation time of input is $5 \mathrm{~ms}$, the cutoff frequency is much lower compared to that of the original Eyal's model. Increasing the correlation time, we can reproduce high bandwidth encoding. But the resonance peak is shifted to the lower frequency. In $\mathrm{B}$, the firing rate is fixed to be around $5 \mathrm{~Hz}$. Here the mean of stimulus is fixed to be $0.005 \mathrm{nA}$ so that the neuron model is in the fluctuation-driven regime.
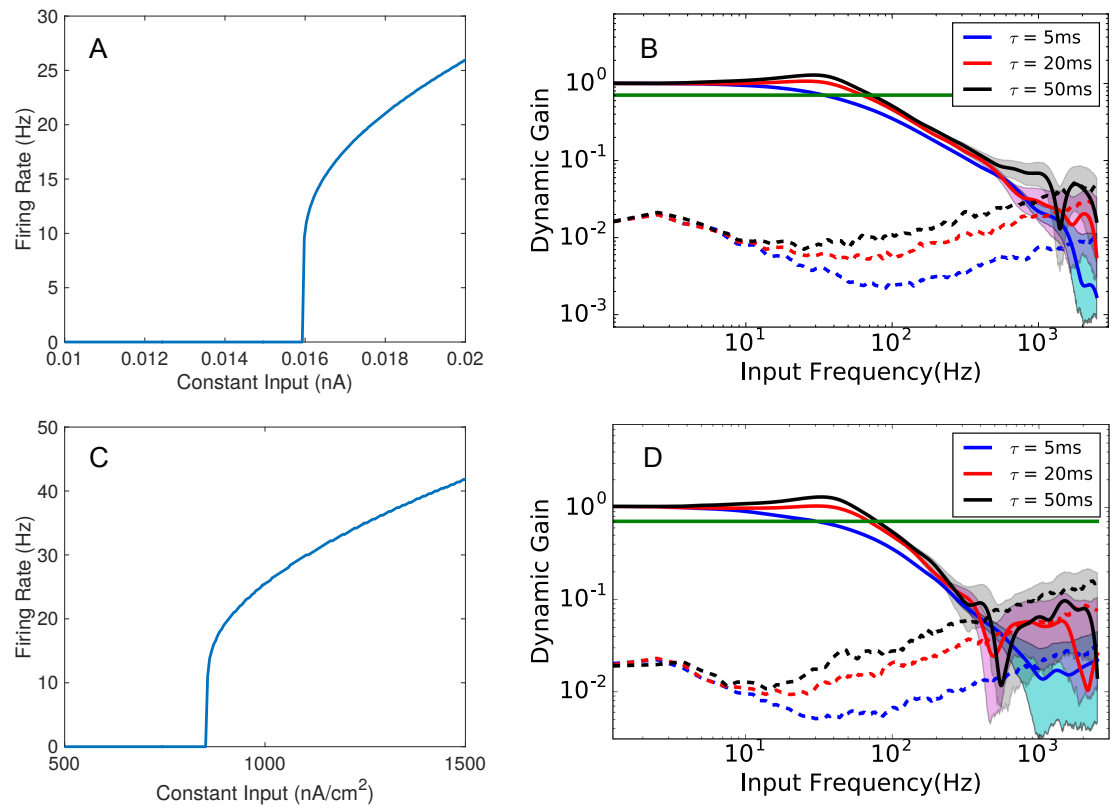

Fig 62. The impact of multi-compartment framework on population encoding in Eyal's mdoel. A, B: The F-I curve and the linear response functions of Eyal's model with a passive soma. C, D: The F-I curve and the linear response functions of Eyal's model written into a single compartment model. The active soma and the separation of the AP initiation site from the soma enhance the discontinuity in the F-I curve and the encoding ability.

In Fig 62 C and D, I wrote Eyal's model into a single compartment model. The dynamics of ion channels are the same as those of original model. To make the neuron model fire properly, the sodium conductance is 
set to be $0.08 S / \mathrm{cm}^{2}$. The potassium conductance is set to be $0.015 \mathrm{~S} / \mathrm{cm}^{2}$. The unit of input current is adjusted from $n A$ to $n A / \mathrm{cm}^{2}$. The firing rate is fixed to be about $5 \mathrm{~Hz}$. The mean is fixed to be $0 \mathrm{nA} / \mathrm{cm}^{2}$. Similar as the multi-compartment model with a passive soma, the high frequency repetitive firing ability is reduced. The high bandwidth encoding can be realized when the correlation time of input is large enough. So from these two models we can see that the multi-compartment model framework does make a contribution to the high frequency repetitive firing. Having an active soma enhances the ability of high frequency repetitive firing for the neuron model. This is particular effective for the cutoff frequency of the linear response functions generated with short correlation time stimulus. For the gain curves generated with large correlation time stimulus, the main impact is on the peak of resonance frequency.
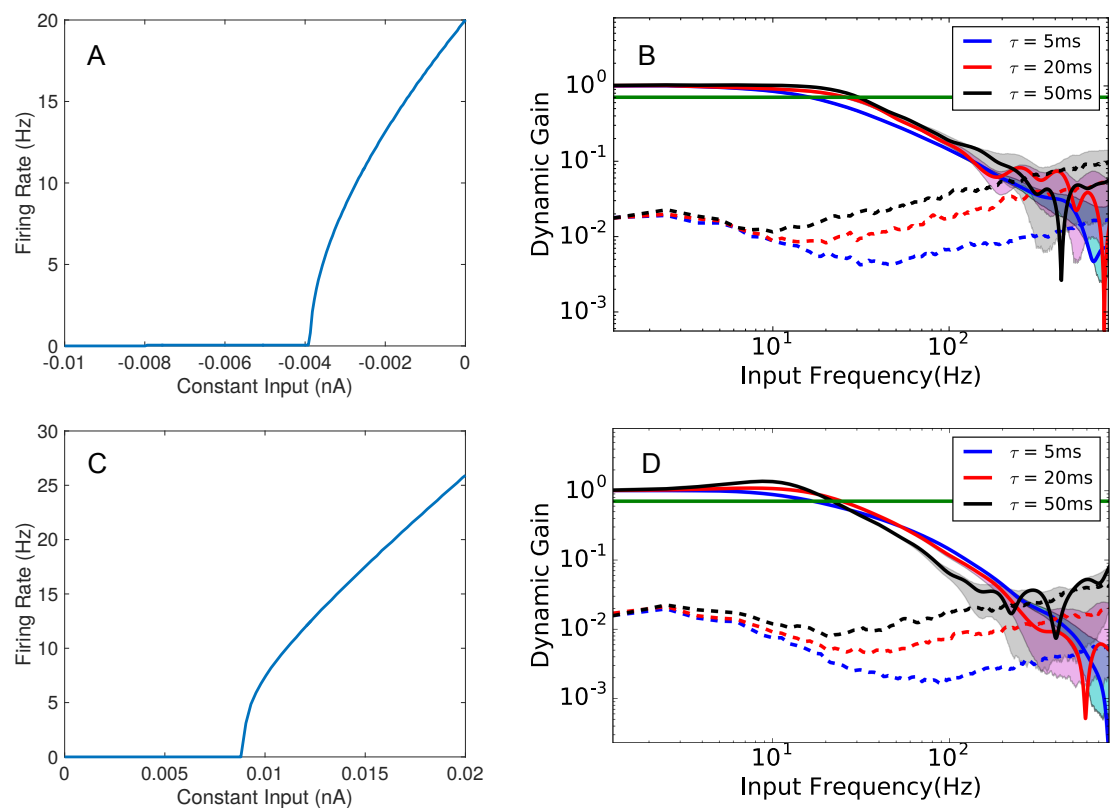

Fig 63. The impact of sodium-potassium AP generation mechanisms on population encoding in Eyal's model. A, B: The F-I curve and the linear response functions of Eyal's model with sodium dynamics shifted $10 \mathrm{mV}$ towards the negative direction. C, D: The F-I curve and the linear response functions of Eyal's model with reset by hand. The relative position of the sodium and potassium dynamics in the voltage axis determines the high frequency repetitive firing in Eyal's model.

In the second part of this section, I will study the impact of sodiumpotassium AP generation mechanisms on population encoding in Eyal's model. Eyal's model is built from the model in 92, 93], in which the sodium dynamics are shifted by $10 \mathrm{mV}$ towards the positive direction in the voltage axis. In Fig $63 \mathrm{~A}$ and $\mathrm{B}$, I shifted back the sodium dynamics. The F-I curve shows that without injecting external current, this neuron model will generate spikes automatically. The rheobase current to trigger spikes is negative. But still we can obtain the F-I curve as from previous models. The stochastic input that generates the aimed firing rate will have a negative mean. For this neuron model, the discontinuity in the F-I curve disappeared. The neuron model turns into a type I model. In the aspect of 
the linear response functions, without high frequency repetitive firing, the cutoff frequencies decrease to 20 to $30 \mathrm{~Hz}$. The dynamic gain in the high frequency is not sensitive to the correlation time of the input, which makes this model similar to the WB model. Here the firing rate is about $5 \mathrm{~Hz}$. The mean of input is fixed to be $-0.006 n A$. In Fig $63 \mathrm{C}$ and D, I write the sodium and potassium dynamics of Eyal's model into Brette's model. The AP initiation point is $47 \mu \mathrm{m}$ from the soma, which is the same as the recording point in Eyal's model. The sodium and potassium conductance at the AP initiation point are the total conductance at the AIS in the original model. Here I reset the voltage to the resting potential by hand once it reaches a threshold. In this way, the oscillation dynamics for AP generation breaks down. When calculating the linear response function, the firing rate is fixed to be $5 \mathrm{~Hz}$. The CV of ISI is fixed to be about 0.85 . The linear response functions have cutoff frequencies around $20 \mathrm{~Hz}$, similar to those of Brette's model. Based on the linear response properties of these two models, we can see that a complete AP generation mechanism is important for high frequency repetitive firing. The relative position of sodium and potassium dynamics on the voltage axis determines the size of discontinuity in the F-I curve.
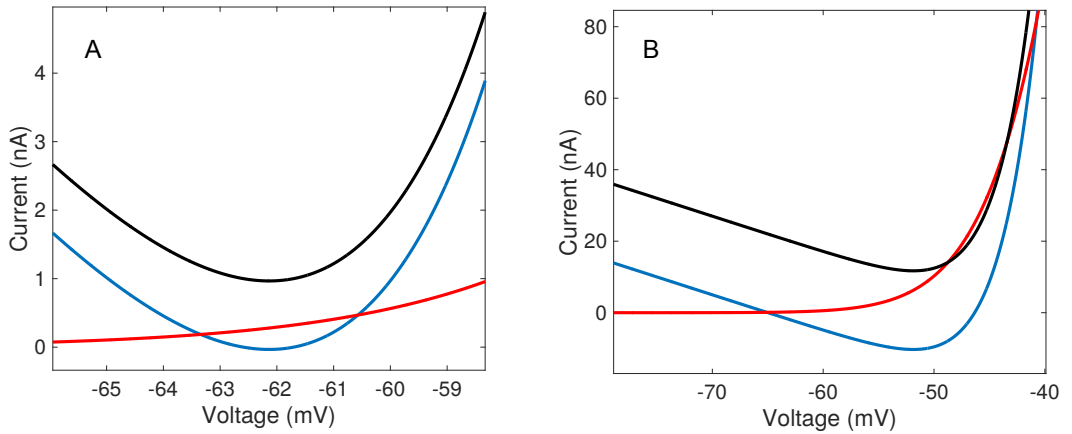

Fig 64. Two types of spike generation bifurcations for different positions of the sodium dynamics on the voltage axis. In $\mathrm{A}$ and $\mathrm{B}$, the blue curves represent the AP initiation dynamics, which is

$g_{L}\left(E_{L}-V\right)+g_{N a} m^{3}\left(E_{N a}-V\right)$. Here the inactivation of sodium channels is taken as 1 . The red curves represent the potassium current dynamics $-g_{K} n^{4}\left(E_{K}-V\right)$. The black curves represents the AP initiation dynamics with a constant external current, which is

$g_{L}\left(E_{L}-V\right)+g_{N a} m^{3}\left(E_{N a}-V\right)+I_{e x t}$. In A, all the parameters and equations are taken from the WB model given in 46. The AP generation bifurcation is a saddle node bifurcation. In $\mathrm{B}$, the sodium dynamics are shifted by $10 \mathrm{mV}$ on the voltage axis towards the positive direction. The AP generation bifurcation is a Hopf bifurcation.

Fig 64 provides an illustration of the change of AP generation bifurcation by shifting the sodium dynamics along the voltage axis. Here I use the parameters and equations from WB model given in [46] as an example. For the neuron equation:

$$
c_{m} \frac{d V}{d t}=g_{L}\left(E_{L}-V\right)+g_{N a} m^{3} h\left(E_{N a}-V\right)+g_{K} n^{4}\left(E_{K}-V\right)+I_{e x t}
$$

I take $g_{L}\left(E_{L}-V\right)+g_{N a} m_{\infty}^{3}\left(E_{N a}-V\right)+I_{e x t}$ over $V$ and $-g_{K} n_{\infty}^{4}\left(E_{K}-V\right)$ over $V$ as two nullclines. The inactivation variable $h$ is assumed to be 1 at low 
voltages. In A, with the original sodium dynamics, there are two intersection points between two nullclines if the external current is 0. Increasing the external current will lead to a saddle node bifurcation which makes the neuron start to generate spikes. In B, the sodium dynamics are shifted by $10 \mathrm{mV}$ in the positive direction. When increasing the external current, the stable fixed point is moved to the right and becomes an unstable fixed point. The Hopf bifurcation happens for the spike generation. So we can see that by changing the relative position of sodium and potassium dynamics along the voltage axis, the neuron type is changed from a type I neuron to a type II neuron, which makes it possible to generate high frequency repetitive firing. 


\section{Summary}

In this thesis, I studied the impact of neuron morphology and biophysics on population encoding. I first introduced simulation methods for calculating the linear response functions which quantify the encoding ability. I showed that the Fourier transform method and the vector strength method provide linear response curves which fit with each other. I also proposed that the uncertainty of time delay between the spike initiation voltage and the spike detection voltage act as a low pass filter in the linear response function. Without the time delay, the linear response functions are similar to those of LIF-like neuron model. Then I examined the axonal hypothesis proposed by Brette [48. I found that with a standard sodium activation function, separating the AP initiation site from the soma is not sufficient to reproduce fast population encoding. The sharp spikes seen at the soma do not necessarily imply high bandwidth encoding. To realize fast population encoding, removing slowly activated sodium current before the AP initiation is also required. This suggests that high voltage sensitivity of sodium activation dynamics might be important for fast population encoding.

The idea of high voltage sensitivity is complementary to the idea of cooperative gating of sodium channels proposed in 46 . Cooperative gating of sodium channels implies that sodium channels will be activated avalanchelike once the voltage is large enough. When the voltage value is low, the activation dynamics of cooperative gating sodium channels can be approximated with that of independent sodium channels. High voltage sensitivity of sodium channels points out that the activation ratio of sodium channels before AP initiation should be smaller than that provided in the standard sodium activation function. The slow sodium activation is usually merged in the low voltage fluctuations dominated by the stochastic stimulus, which makes it seem negligible for population encoding. However, I found that it can play an important role in determining the high bandwidth encoding. So it remains to be further examined in the sodium activation dynamics of cortical neurons. One possibility is that the current function form for the sodium activation dynamics does not capture the sodium activation dynamics of individual sodium channels. Another possibility is that some other types of currents need to be included in the model.

In the following chapter, I examined the encoding ability of the cooperative gating model [46]. Based on the given parameters including ratio of cooperative gating sodium channels and strength of cooperativity, I did not manage to realize fast population encoding. The cooperative gating dynamics did not resolve the problem of slow activation near AP threshold. Increasing the ratio or the strength of cooperativity failed to realize high bandwidth encoding as well. One additional implication from the examination of the cooperative gating model is on the inactivation of sodium channels. High voltage sensitivity of sodium activation implies a large amount of activated sodium channels once some specific voltage is reached. When finishing generating a spike, if the sodium channels are deinactivated before this specific voltage, the large amount of sodium current will prevent the voltage from being reset properly. Instead, the voltage will be locked at some intermediate value and not generating spikes anymore. Avoiding this phenomenon required that a sufficient amount of sodium channels blocked until the voltage is below the sodium activation voltage. The inactivation dynamics in WB model fail to capture this feature. To fix this problem, a 
better description of the inactivation dynamics remains for further study. Here in my work, one way found to fix this problem was to introduce cooperative gating of deinactivation of sodium channels. Sodium channels then are blocked until the voltage is low enough. Then the sodium channels are deinactivated together. In this way, the neuron model can fire properly.

In the last chapter, I studied the underlying mechanisms of the multicompartment model used in [49] for realizing high bandwidth encoding. Without requiring high voltage sensitivity on sodium activation dynamics, the cutoff frequency of its linear response functions is above 100Hz. Here I reproduced high bandwidth encoding, and showed that the dynamic gain in the high frequency regime was not sensitive to the correlation time of stimulus. This indicates that the neuron model does not fully reproduce fast population encoding. Then I showed that the F-I curve of the neuron model had a discontinuity at rheobase current. The high bandwidth encoding is realized with high frequency repetitive firing which selectively enhances the dynamic gain in the high frequency region. Comparing with the neuron models that have low cutoff frequencies in their linear response functions, I showed that an active soma and the relative position of sodium and potassium dynamics along the voltage axis determine the high frequency repetitive firing ability of the neuron model.

In this thesis, I used the linear response function to quantify the population encoding ability of different neuron models. From the view of neural network, it is still unclear how the encoding ability of cortical neurons impact the information transfer and information processing in the neural network. Especially in a network with both excitatory (type I) and inhibitory (type II) neuron. It would be interesting to study the mixed networks in the future. 


\section{Acknowledgment}

I would like to thank China Scholarship Council for providing me the scholarship for my PhD study and research. I would like to thank my families and my girl friend Miss Lin for the mental support and encouragement for all these days.

I would like to thank Prof. Fred Wolf for providing me such an interesting topic as my first step into the field of computational neuroscience, and guiding me through the exploration of the scientific questions. I would like to thank Dr. Andreas Neef for fruitful discussions and collaborations on my $\mathrm{PhD}$ projects. I would like to thank Prof. Theo Geisel for organizing an energetic institute with people from different backgrounds doing science together. I would like to thank Prof. Joerg Enderlein and Prof. Robert Guetig for being my thesis committee members and providing me suggestions and discussions for the PhD studies.

I would like to thank Chepe and Tatjana for great help and advice on my research and future path planning. I would like to thank Jonas for being a great office member discussing scientific questions and maintaining harmonic working environment. I would like to thank Matthias, Nico, Julian, Eric, Barbara, Alex, Agostina, Frank, Max, Guillaume, Tureiti for the discussions and help on my progress results. I would like to thank Joao, Lucas, George and all the others in our institute for being nice and friendly colleagues.

I would like to thank the secretaries Vika, Ayse, Christina, Rebecca and Yvonne for all the help on administrations and documents. I would like to thank Yorck, Denny and Hecke for technical support. 


\section{References}

1. Huxley F. The dual effect of membrane potential on sodium conductance in the giant axon of Loligo. The Journal of Physiology. 1952;116:497-506.

2. Hodgkin AL, Huxley AF, Katz B. Measurement of current-voltage relations in the membrane of the giant axon of Loligo. The Journal of Physiology. 1952;116:424-448.

3. Hodgkin AL, Huxley AF. Currents carried by sodium and potassium ions through the membrane of the giant axon of Loligo. The Journal of Physiology. 1952;116:449-472.

4. Huxley AF. The components of membrane conductance in L411mV. The Journal of Physiology. 1952;116:473-496.

5. Hodgkin aL, Huxley aF. A quantitative description of membrane current and its application to conduction and excitation in nerve. Bulletin of Mathematical Biology. 1990;52:25-71. doi:10.1016/S00928240(05)80004-7.

6. Xiao-Jing Wang and György Buzsáki. Gamma oscillation by synaptic inhibition in a hippocampal interneuronal network model. The Journal of Neuroscience. 1996;16(20):6402-6413. doi:citeulike-articleid:134404.

7. Naundorf B, Wolf F, Volgushev M. Unique features of action potential initiation in cortical neurons. Nature. 2006;440(April):1060-1063. doi:10.1038/nature04610.

8. McCormick DA, Shu Y, Yu Y. Neurophysiology: Hodgkin and Huxley model - still standing? Nature. 2007;445(7123):2006-2007. doi:10.1038/nature05523.

9. Ilin V, Malyshev A, Wolf F, Volgushev M. Fast computations in cortical ensembles require rapid initiation of action potentials. Journal of Neuroscience. 2013;33(6):2281-2292. doi:10.1523/JNEUROSCI.077112.2013 .

10. Thorpe S, Fize D, Marlot C. Speed of processing in the human visual system. Nature. 1996;381(6582):520-522. doi:10.1038/381520a0.

11. Paré M, Munoz DP. Saccadic reaction time in the monkey: advanced preparation of oculomotor programs is primarily responsible for express saccade occurrence. Journal of Neurophysiology. 1996;76(6):3666-3681.

12. Stanford TR, Shankar S, Massoglia DP, Costello MG, Salinas E. Perceptual decision making in less than 30 milliseconds. Nature Neuroscience. 2010;13(3):379-385. doi:10.1038/nn.2485.

13. Johansson RS, Birznieks I. First spikes in ensembles of human tactile afferents code complex spatial fingertip events. Nature Neuroscience. 2004;7(2):170-177. doi:10.1038/nn1177.

14. VanRullen R, Guyonneau R, Thorpe SJ. Spike times make sense. Trends in Neurosciences. 2005;28(1):1-4. doi:10.1016/j.tins.2004.10.010. 
15. Softky WR, Koch C. The highly irregular firing of cortical cells is inconsistent with temporal integration of random EPSPs. The Journal of Neuroscience. 1993;13(1):334-50.

16. Shinomoto S, Sakai Y, Funahashi S. The Ornstein-Uhlenbeck process does not reproduce spiking statistics of neurons in prefrontal cortex. Neural Computation. 1999;11:935-951. doi:10.1162/089976699300016511.

17. Vogels R, Spileers W, Orban GA. The response variability of striate cortical neurons in the behaving monkey. Experimental Brain Research. 1989; p. 28-32.

18. Tolhurst DJ, Movshon JA, Dean AF. The statistical reliability of signals in single neurons in cat and monkey visual cortex. Vision Research. 1983;23(8):775-785. doi:10.1016/0042-6989(83)90200-6.

19. Schiller PH, Finlay BL, Volman SF. Short-term response variability of monkey striate neurons. Brain Research. 1976;105(2):347-9. doi:00068993(76)90432-7 [pii].

20. Britten KH, Shadlen MN, Newsome WT, Movshon JA. Responses of neurons in macaque MT to stochiastic motion signals. Visual Neuroscience. 1993;(10):1157-1169.

21. Shadlen MN, Newsome WT. Noise, neural codes and cortical organization. Current Opinion in Neurobiology. 1994;4(4):569-579. doi:10.1016/0959-4388(94)90059-0.

22. Shadlen MN, Newsome WT. The variable discharge of cortical neurons: implications for connectivity, computation, and information coding. The Journal of Neuroscience. 1998;18(10):3870-96. doi:02706474/98/183870-2705.00/0.

23. Anderson JS. The contribution of noise to contrast invariance of orientation tuning in cat visual cortex. Science. 2000;290(5498):19681972. doi:10.1126/science.290.5498.1968.

24. Cowan RL, Wilson CJ. Spontaneous firing patterns and axonal projections of single corticostriatal neurons in the rat medial agranular cortex. Journal of Neurophysiology. 1994;71(1):17-32. doi:10.1152/jn.1994.71.1.17.

25. Stern EA, Kincaid AE, Wilson CJ. Spontaneous subthreshold membrane potential fluctuations and action potential variability of rat corticostriatal and striatal neurons in vivo. Journal of Neurophysiology. 1997;77(4):1697-1715.

26. Gawne TJ, Richmond BJ. How independent are the messages carried by adjacent inferior temporal cortical neurons? The Journal of Neuroscience. 1993;13(7):2758-71.

27. Zohary E, Shadlen MN, Newsome WT. Correlated neuronal discharge rate and its implications for psychophysical performance. Nature. 1994;370(6485):140-143. doi:10.1038/370140a0. 
28. Lee D, Port NL, Kruse W, Georgopoulos aP. Variability and correlated noise in the discharge of neurons in motor and parietal areas of the primate cortex. The Journal of Neuroscience. 1998;18(3):1161-70. doi:citeulike-article-id:90465.

29. Bair W, Zohary E, Newsome WT. Correlated firing in macaque visual area MT: time scales and relationship to behavior. The Journal of Neuroscience. 2001;21(5):1676-97. doi:21/5/1676 [pii].

30. Constantinidis C, Goldman-Rakic PS. Correlated discharges among putative pyramidal neurons and interneurons in the primate prefrontal cortex. Journal of Neurophysiology. 2002;88(6):3487-97. doi:10.1152/jn.00188.2002.

31. Ecker AS, Berens P, Keliris GA, Bethge M, Logothetis NK, Tolias AS. Decorrelated neuronal firing in cortical microcircuits. Science. 2010;327(5965):584-587. doi:10.1126/science.1179867.

32. Renart A, Rocha JD, Bartho P, Hollender L, Parga N, Reyes A, et al. The asynchronous state in cortical circuits. Science. 2010;327(January):587-591. doi:10.1126/science.1179850.

33. Köndgen H, Geisler C, Fusi S, Wang XJ, Lüscher HR, Giugliano M. The dynamical response properties of neocortical neurons to temporally modulated noisy inputs in vitro. Cerebral Cortex. 2008;18(September):2086-2097. doi:10.1093/cercor/bhm235.

34. Boucsein C, Tetzlaff T, Meier R, Aertsen A, Naundorf B. Dynamical response properties of neocortical neuron ensembles: multiplicative versus additive noise. The Journal of Neuroscience. 2009;29(4):10061010. doi:10.1523/JNEUROSCI.3424-08.2009.

35. Higgs MH, Spain WJ. Conditional bursting enhances resonant firing in neocortical layer 2-3 pyramidal neurons. The Journal of Neuroscience. 2009;29(5):1285-99. doi:10.1523/JNEUROSCI.3728-08.2009.

36. Tchumatchenko T, Malyshev A, Wolf F, Volgushev M. Ultrafast population encoding by cortical neurons. Journal of Neuroscience. 2011;31(34):12171-12179. doi:10.1523/JNEUROSCI.2182-11.2011.

37. Knight BW. The relationship between the firing rate of a single neuron and the level of activity in a population of neurons: experimental evidence for resonant enhancement in the population response. The Journal of General Physiology. 1972;59(6):767-778. doi:10.1085/jgp.59.6.767.

38. Knight BW. Dynamics of encoding in a population of neurons. The Journal of General Physiology. 1972;59(6):734-766. doi:10.1085/jgp.59.6.734.

39. Brunel N, Chance FS, Fourcaud N, Abbott LF. Effects of synaptic noise and filtering on the frequency response of spiking neurons. Physical Review Letters. 2001;86(10):2186-9.

40. Lindner B, Schimansky-Geier L. Transmission of noise coded versus additive signals through a neuronal ensemble. Physical Review Letters. 2001;86(14):2934-2937. doi:10.1103/PhysRevLett.86.2934. 
41. Fourcaud-Trocmé N, Hansel D, van Vreeswijk C, Brunel N. How spike generation mechanisms determine the neuronal response to fluctuating inputs. The Journal of Neuroscience. 2003;23(37):1162840 .

42. Fourcaud-Trocmé N, Brunel N. Dynamics of the instantaneous firing rate in response to changes in input statistics. Journal of Computational Neuroscience. 2005;18(3):311-321. doi:10.1007/s10827-005$0337-8$.

43. Naundorf B, Geisel T, Wolf F. Action potential onset dynamics and the response speed of neuronal populations. Journal of Computational Neuroscience. 2005;18(3):297-309. doi:10.1007/s10827-005-0329-8.

44. Tchumatchenko T, Wolf F. Representation of dynamical stimuli in populations of threshold neurons. PLoS Computational Biology. 2011;7(10). doi:10.1371/journal.pcbi.1002239.

45. Wei W, Wolf F. Spike onset dynamics and response speed in neuronal populations. Physical Review Letters. 2011;106(February):1-4. doi:10.1103/PhysRevLett.106.088102.

46. Huang M, Volgushev M, Wolf F. A small fraction of strongly cooperative sodium channels boosts neuronal encoding of high frequencies. PLoS ONE. 2012;7(5). doi:10.1371/journal.pone.0037629.

47. Puelma Touzel M, Wolf F. Complete firing-rate response of neurons with complex intrinsic dynamics. PLoS Computational Biology. 2015;11(12):1-43. doi:10.1371/journal.pcbi.1004636.

48. Brette R. Sharpness of spike initiation in neurons explained by compartmentalization. PLoS computational biology. 2013;9(12):e1003338. doi:10.1371/journal.pcbi.1003338.

49. Eyal G, Mansvelder HD, de Kock CPJ, Segev I. Dendrites impact the encoding capabilities of the axon. The Journal of Neuroscience. 2014;34(24):8063-71. doi:10.1523/JNEUROSCI.5431-13.2014.

50. Brette R. What is the most realistic single-compartment model of spike initiation? PLoS Computational Biology. 2015;11(4):1-13. doi:10.1371/journal.pcbi.1004114.

51. Telenczuk M, Fontaine B, Brette R. The basis of sharp spike onset in standard biophysical models. PLoS ONE. 2017;12(4):1-27. doi:10.1371/journal.pone.0175362.

52. Ostojic S, Szapiro G, Schwartz E, Barbour B, Brunel N, Hakim V. Neuronal morphology generates high-frequency firing resonance. Journal of Neuroscience. 2015;35(18):7056-7068. doi:10.1523/JNEUROSCI.3924-14.2015.

53. Öz P, Huang M, Wolf F. Action potential initiation in a multicompartmental model with cooperatively gating $\mathrm{Na}$ channels in the axon initial segment. Journal of Computational Neuroscience. 2015;39(1):63-75. doi:10.1007/s10827-015-0561-9. 
54. Badel L. Firing statistics and correlations in spiking neurons: A levelcrossing approach. Physical Review E - Statistical, Nonlinear, and Soft Matter Physics. 2011;84(4). doi:10.1103/PhysRevE.84.041919.

55. Kapanipathi P, Orlandi F, Sheth A, Passant A. Personalized filtering of the Twitter stream. CEUR Workshop Proceedings. 2011;781(20):613. doi:10.1523/JNEUROSCI.0552-10.2010.

56. Palmer LM, Clark Ba, Gründemann J, Roth A, Stuart GJ, Häusser M. Initiation of simple and complex spikes in cerebellar Purkinje cells. The Journal of Physiology. 2010;588(Pt 10):1709-17. doi:10.1113/jphysiol.2010.188300.

57. Clark BD, Goldberg EM, Rudy B. Electrogenic tuning of the axon initial segment. The Neuroscientist. 2009;15(6):651-668. doi:10.1177/1073858409341973.

58. Coombs JS, Curtis DR, Eccles JC. The interpretation of spike potentials of motoneurones. The Journal of Physiology. 1957;139(2):198231. doi:10.1113/jphysiol.1957.sp005887.

59. Stuart G, Spruston N, Sakmann B, Hausser M. Action potential initiation and back propagation in neurons of the mammalian central nervous system. Trends in Neurosciences. 1997;20(3):125-131. doi:10.1016/S0166-2236(96)10075-8.

60. Shu Y, Duque A, Yu Y, Haider B, McCormick DA. Properties of action-potential initiation in neocortical pyramidal cells: evidence from whole cell axon recordings. Journal of Neurophysiology. 2007;97(1):746-760. doi:10.1152/jn.00922.2006.

61. Boudkkazi S, Carlier E, Ankri N, Caillard O, Giraud P, FronzaroliMolinieres L, et al. Release-dependent variations in synaptic latency: a putative code for short- and long-term synaptic dynamics. Neuron. 2007;56(6):1048-1060. doi:10.1016/j.neuron.2007.10.037.

62. Palmer LM, Stuart GJ. Site of action potential initiation in layer 5 pyramidal neurons. The Journal of Neuroscience. 2006;26(6):1854-63. doi:10.1523/JNEUROSCI.4812-05.2006.

63. Yu Y, Shu Y, McCormick DA. Cortical action potential backpropagation explains spike threshold variability and rapid-onset kinetics. The Journal of Neuroscience. 2008;28(29):7260-72. doi:10.1523/JNEUROSCI.1613-08.2008.

64. Colbert CM, Johnston D. Axonal action-potential initiation and Na+ channel densities in the soma and axon initial segment of subicular pyramidal neurons. The Journal of Neuroscience. 1996;16(21):667686. doi: 8824308 .

65. Khaliq ZM. Relative contributions of axonal and somatic Na channels to action potential initiation in cerebellar purkinje neurons. Journal of Neuroscience. 2006;26(7):1935-1944. doi:10.1523/JNEUROSCI.466405.2006.

66. Hu W, Tian C, Li T, Yang M, Hou H, Shu Y. Distinct contributions of $\mathrm{Na}(\mathrm{v}) 1.6$ and $\mathrm{Na}(\mathrm{v}) 1.2$ in action potential initiation and backpropagation. Nature Neuroscience. 2009;12(8):996-1002. doi:10.1038/nn.2359. 
67. Kole MHP, Letzkus JJ, Stuart GJ. Axon Initial Segment Kv1 Channels Control Axonal Action Potential Waveform and Synaptic Efficacy. Neuron. 2007;55(4):633-647. doi:10.1016/j.neuron.2007.07.031.

68. Kole MHP, Ilschner SU, Kampa BM, Williams SR, Ruben PC, Stuart GJ. Action potential generation requires a high sodium channel density in the axon initial segment. Nature Neuroscience. 2008;11(2):17886. doi: $10.1038 / \mathrm{nn} 2040$.

69. Fleidervish IA, Lasser-Ross N, Gutnick MJ, Ross WN. Na+ imaging reveals little difference in action potential-evoked $\mathrm{Na}+$ influx between axon and soma. Nature Neuroscience. 2010;13(7):852-60. doi:10.1038/nn.2574.

70. Lazarov E, Dannemeyer M, Feulner B, Enderlein J, Gutnick MJ, Wolf $\mathrm{F}$, et al. Axonal spike initiation can be maintained with low axonal $\mathrm{Na}$ channel density, but temporal precision of spiking is lost;.

71. Debanne D, Campanac E, Bialowas A, Edmond C, Alcaraz G. Axon physiology. Physiological Reviews. 2011; p. 555-602. doi:10.1152/physrev.00048.2009.

72. Undrovinas AI, Fleidervish IA, Makielski JC. Inward sodium current at resting potentials in single cardiac myocytes induced by the ischemic metabolite lysophosphatidylcholine. Circulation research. 1992;71(5):1231-1241. doi:10.1161/01.RES.71.5.1231.

73. Molina ML, Barrera FN, Fernández AM, Poveda JA, Renart ML, Encinar JA, et al. Clustering and coupled gating modulate the activity in KcsA, a potassium channel model. Journal of Biological Chemistry. 2006;281(27):18837-18848. doi:10.1074/jbc.M600342200.

74. Marx SO, Gaburjakova J, Gaburjakova M, Henrikson C, Ondrias K, Marks AR. Coupled Gating Between Cardiac Calcium Release Channels (Ryanodine Receptors). Circulation Research. 2001;88(11):11511158. doi:10.1161/hh1101.091268.

75. Keleshian AM, Edeson RO, Liu GJ, Madsen BW. Evidence for cooperativity between nicotinic acetylcholine receptors in patch clamp records. Biophysical Journal. 2000;78(1):1-12. doi:10.1016/S00063495(00)76568-4.

76. Brunel N, Hakim V, Richardson MJE. Single neuron dynamics and computation. Current Opinion in Neurobiology. 2014;25:149-155. doi:10.1016/j.conb.2014.01.005.

77. London M, Häusser M. Dendritic computation. Annual Review of Neuroscience. 2005;28:503-32. doi:10.1146/annurev.neuro.28.061604.135703.

78. Cash S, Yuste R. Linear summation of excitatory inputs by CA1 pyramidal neurons. Neuron. 1999;22(2):383-394. doi:10.1016/S08966273(00)81098-3.

79. Brunel N, Hakim V, Isope P, Nadal JP, Barbour B. Optimal information storage and the distribution of synaptic weights: Perceptron versus Purkinje cell. Neuron. 2004;43(5):745-757. doi:10.1016/j.neuron.2004.08.023. 
80. Rall W. Theoretical significance of dendritic trees for neural inputoutput relations. Neural Theory and Modeling; p. 73-97.

81. Rall W, Burke R, Smith T, Nelson P, Frank K. Dendritic location of synapses and possible mechanisms for the monosynaptic EPSP in motoneurons. Journal of Neurophysiology. 1967;30(5):1169-1193. doi:4293410.

82. Liu G. Local structural balance and functional interaction of excitatory and inhibitory synapses in hippocampal dendrites. Nature Neuroscience. 2004;7(4):373-379. doi:10.1038/nn1206.

83. Migliore M, Shepherd GM. Emerging rules for the distributions of active dendritic conductances. Nature Reviews Neuroscience. 2002;3(5):362-370. doi:10.1038/nrn810.

84. Cook EP, Johnston D. Voltage-dependent properties of dendrites that eliminate location-dependent variability of synaptic input. Journal of Neurophysiology. 1999;81(2):535-543.

85. Cook EP, Johnston D. Active dendrites reduce location-dependent variability of synaptic input trains. Journal of Neurophysiology. 1997;78(4):2116-2128.

86. Larkum M. A cellular mechanism for cortical associations: An organizing principle for the cerebral cortex. Trends in Neurosciences. 2013;36(3):141-151. doi:10.1016/j.tins.2012.11.006.

87. Major G, Larkum ME, Schiller J. Active properties of neocortical pyramidal neuron dendrites. Annual Review of Neuroscience. 2013;36(1):1-24. doi:10.1146/annurev-neuro-062111-150343.

88. Smith SL, Smith IT, Branco T, Häusser M. Dendritic spikes enhance stimulus selectivity in cortical neurons in vivo. Nature. 2013;503(7474):115-20. doi:10.1038/nature12600.

89. Carnevale NT, Hines ML. The NEURON book. Cambridge University Press; 2006.

90. Stuart GJ, Sakmann B. Active propagation of somatic action potentials into neocortical pyramidal cell dendrites. Nature. 1994;367(6458):69-72. doi:10.1038/367069a0.

91. Silberberg G, Bethge M, Markram H, Pawelzik K, Tsodyks M. Dynamics of population rate codes in ensembles of neocortical neurons. Journal of Neurophysiology. 2004;91:704-709. doi:10.1152/jn.00415.2003.

92. Mainen ZF, Joerges J, Huguenard JR, Sejnowski TJ. A model of spike initiation in neocortical pyramidal neurons. Neuron. 1995;15(6):14271439. doi:10.1016/0896-6273(95)90020-9.

93. Mainen ZF, Sejnowski TJ. Influence of dendritic structure on firing pattern in model neocortical neurons. Nature. 1996;382(6589):363366. doi:10.1038/382363a0. 